\begin{abstract}
Title of dissertation: TOPOLOGICAL EDGE STATES IN SILICON PHOTONICS

Sunil Mittal

Doctor of Philosophy, 2014
Dissertation directed by: Professor Mohammad Hafezi
Department of Electrical and Computer Engineering University of Maryland, College Park

Under the influence of a magnetic field, at low temperatures, charged particles confined in two-dimensional systems exhibit a remarkable range of macroscopic quantum phenomena such as the quantum Hall effects. A hallmark of these phenomena is the presence of unidirectional, topologically robust edge states - states which are confined to the edge of the system. It is, in principle, possible to engineer a synthetic magnetic field for photons and hence achieve photonic analogs of the robust electronic edge states. Investigating photonic edge states is interesting from a fundamental perspective of studying photonic transport in the presence of a gauge field and also for its application in classical and quantum information processing.

In this thesis, we present the implementation of a synthetic magnetic field for photons and our observation of topological edge states in a two-dimensional lattice of coupled ring resonators, fabricated using CMOS-compatible silicon-oninsulator technology. We qualitatively show the robustness of edge states against deliberately induced lattice defects. We then analyze the statistics of transport 
measurements (transmission and delay) made on a number of different devices and quantitatively verify the robustness of edge states against lattice disorder. Using Wigner delay-time distribution, we show that localization is suppressed in the edge states. Furthermore, to unequivocally establish the non-trivial topological nature of edge states, we compare their transmission to a topologically trivial one dimensional system of coupled ring resonators and demonstrate that the edge states achieve higher transmission.

Moreover, for photonic analogs of the quantum Hall effect, the winding number - a topologically invariant integer which characterizes edge states - is quantized, analogous to quantization of conductivity in electronic systems. We measure the winding number of the edge states in our system. Finally, we investigate the effect of nonlinear interactions in silicon ring resonators, on the stability of edge states. We show that the presence of a strong pump can result in a significant decrease in the transmission through edge states. 


\title{
TOPOLOGICAL EDGE STATES IN SILICON PHOTONICS
}

\author{
by
}

Sunil Mittal

Dissertation submitted to the Faculty of the Graduate School of the University of Maryland, College Park in partial fulfillment of the requirements for the degree of

Doctor of Philosophy

2014

Advisory Committee:

Professor Mohammad Hafezi, Advisor/Chair

Professor Alan Migdall, Co-Advisor

Professor Thomas Murphy

Professor Edo Waks

Professor Rajarshi Roy 
(c) Copyright by

Sunil Mittal

2014 
Dedicated to My Family 83 Teachers 


\section{Acknowledgments}

This thesis would be incomplete without mentioning my association with the people who made it possible for me to sail through this journey.

My advisors, Prof. Mohammad Hafezi and Prof. Alan Migdall, have been a continuous source of invaluable guidance, support and encouragement. Whatever I learned about quantum Hall effects, I owe it to Mohammad. His effortless ways of explaining complicated things have been instrumental in achieving this milestone. I will always try and imbibe his 'step-by-step' approach towards solving intricate scientific problems. Alan's infectious enthusiasm and scientific attitude has always inspired me to seek an intuitive understanding of science. Discussions with him never failed to bear rich dividends. His energetic temperament while working in the lab is unmatched, I never saw him tired and exhausted.

I am indebted to Prof. Jacob Taylor for all the insightful discussions. His clear perception, intellectual contributions and expertise in scientific writing and presentation have been immensely valuable. I have been fortunate to have Dr. Jingyun Fan around in the lab. He was always available for any kind of help in setting up the experiments. His experience with most of the equipments in the lab ensured that I do not have to struggle working with them.

I would like to extend sincere thanks to my Committee members Prof. Thomas Murphy, Prof. Edo Waks and Prof. Rajarshi Roy for their invaluable time and useful feedback. I would also like to thank Prof. Jeremy Munday who served on my Proposal Committee. 
I am also grateful to members of Alan Migdall's group at NIST including Elohim Becerra, Joffrey Peters, Sergey Polyakov, Boris Glebov, Elizabeth Goldschmidt, and Yu-Hsiang Cheng, who provided a cordial atmosphere in the lab. I owe a heartfelt thanks to my friends at UMD, especially, Shilpi, Ajay, Jeyran and Ryan. They were always around to share the joys and sorrows during my stay at UMD.

My deepest gratitude goes to my family whose resilience and colossal amount of endurance made me stick through the thick and thin during this journey. My parents' faith in my decisions gave me the strength to execute them. A special thanks is due to Manisha who has been always a dear friend. Last but not the least, I would also like to thank my wife, Kanchan, who has been on an endless trail of cooking ever since we got married !! 


\section{Table of Contents}

List of Figures vii

List of Abbreviations $\quad$ ix

1 Introduction 1

2 The Quantum Hall Effect 6

2.1 The Classical Hall Effect . . . . . . . . . . . . . . . . . . . . . . 8

2.1.1 Electron Motion in a Magnetic Field . . . . . . . . . . . . . 9

2.1.2 Electron Motion in Electric and Magnetic Fields . . . . . . . . 10

2.2 The Quantum Hall Effect . . . . . . . . . . . . . . . . . . 11

2.2.1 Electron Motion in a Magnetic Field . . . . . . . . . . . . . . 12

2.2.2 Electron Motion in Electric and Magnetic Fields . . . . . . . . 17

2.3 Robustness of Quantization and Gauge Invariance . . . . . . . . . . . 19

2.4 The Tight-Binding Model . . . . . . . . . . . . . . . . . . . . 22

2.4.1 Hofstadter Spectrum and Edge States . . . . . . . . . . . . 23

2.4.2 The Dispersion Relation . . . . . . . . . . . . . . . 26

2.5 Summary . . . . . . . . . . . . . . . . . 26

3 Ring Resonator 28

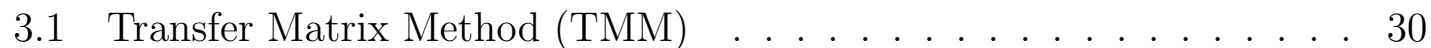

3.2 Single Mode Approximation (SMA) . . . . . . . . . . . . . . . . . . 34

3.3 Equivalence between TMM and SMA . . . . . . . . . . . . . . 37

3.4 Finesse and Quality Factor of the Resonator . . . . . . . . . . . . 41

3.5 Summary . . . . . . . . . . . . . . . . . . . 42

4 Synthetic Magnetic Field for Photons 44

4.1 Coupled Ring Resonators . . . . . . . . . . . . . . . . . . . . . . . . . 44

4.1.1 Tight-Binding Model without Magnetic Field . . . . . . . . . 47

4.1.2 Magnetic Field for Photons . . . . . . . . . . . . . . . 51

4.2 A Finite Lattice - Transmission, Delay \& Edge States . . . . . . . . . 54

4.3 Spin Degree of Freedom . . . . . . . . . . . . . . . . 60

4.4 Edge States as Robust Optical Delay Lines . . . . . . . . . . . . . . . 62 
4.5 Transfer Matrix Simulations . . . . . . . . . . . . . . . 65

4.6 Summary . . . . . . . . . . . . . . . . . . . 71

5 Robust Transport of Photons through Edge States 72

5.1 Device Fabrication . . . . . . . . . . . . . . . . . . . 72

5.1 .1 Lithography . . . . . . . . . . . . . . . . . 74

5.1 .2 Coupling Light to Waveguides . . . . . . . . . . . . . 76

5.2 Device Design and Spectrum Measurements . . . . . . . . . . . . . 77

5.2.1 The ADF Design and Spectrum . . . . . . . . . . . . . 79

5.2.2 The 2D Lattice Design and Spectrum . . . . . . . . . . . 83

5.3 Imaging the Path of Light . . . . . . . . . . . . . . . 86

5.3.1 CCD Camera Calibration . . . . . . . . . . 86

5.3 .2 Imaging Edge States . . . . . . . . . . . . . . . . . . 87

5.3.3 Robustness of Edge States . . . . . . . . . . . . . . . . . 90

5.4 Robust Transport of Photons . . . . . . . . . . . . . . . . . . 91

5.4.1 Delay Distribution . . . . . . . . . . . . . . . . . 92

5.4.2 Comparison with 1D Array . . . . . . . . . . . . . 93

5.4.3 Disorder Characterization and Numerical Simulations . . . . . 99

5.4.4 Calibration of Spectra and Device Yield . . . . . . . . . . 100

5.5 Summary . . . . . . . . . . . . . . . . . . . . . 102

6 Measuring Winding Number in Photonic Systems 105

6.1 Winding Number of Edge States in a Square Annulus . . . . . . . . . 106

6.2 Winding Number in a Chain of Ring Resonators . . . . . . . . . . . . 114

6.3 Heater Calibration . . . . . . . . . . . . . . . . . . . 115

6.4 Summary . . . . . . . . . . . . . . . . . . . . 117

7 Effect of Silicon Nonlinearities on Edge States 118

7.1 Nonlinear Effects in Silicon Ring Resonators . . . . . . . . . . . . . . 119

7.1.1 Kerr Effect . . . . . . . . . . . . . . . . . . . 120

7.1.2 Two-Photon Absorption . . . . . . . . . . . . . . 121

7.1.3 Free Carriers . . . . . . . . . . . . . . . . . . . . . 122

7.1.4 Thermo-Optic Effects . . . . . . . . . . . . . . . . 124

7.1.5 Modeling . . . . . . . . . . . . . . . . 124

7.2 Experimental Characterization of Nonlinear Parameters . . . . . . . . 126

7.3 Problem of Global Shift . . . . . . . . . . . . . . . . . . . 130

7.4 Effect of Nonlinear Interactions on Edge States . . . . . . . . . . . . 132

7.5 Summary . . . . . . . . . . . . . . . . 134

8 Conclusions and Outlook 135

A Spin Degree of Freedom in a Ring Resonator 138

B Effect of Link Ring on Coupling Rate 143

$\begin{array}{ll}\text { Bibliography } & 147\end{array}$ 


\section{List of Figures}

1.1 The 2D lattice of coupled ring resonators used to implement synthetic magnetic field for photons. . . . . . . . . . . . . . . . 4

2.1 Quantization of Hall resistivity as a function of applied magnetic field. 7

2.2 Classical Hall effect in a 2D conductor. . . . . . . . . . . . . . 8

2.3 Cyclotron motion in a perpendicular magnetic field. . . . . . . . . . 9

2.4 Cyclotron motion of electron superimposed over quantized wavefunction. . . . . . . . . . . . . . . . . 15

2.5 Corbino geometry used to quantization of flux in Hall systems. . . . . 19

2.6 2D lattice used to study the quantum Hall effect. . . . . . . . . . . . 21

2.7 Hofstadter butterfly spectrum for an infinite and finite lattice. . . . . 23

2.8 Dispersion for an infinite lattice. . . . . . . . . . . . . . . 25

3.1 Schematic of an Add-Drop Filter and an All-Pass Filter. . . . . . . . 29

3.2 The TMM and the SMA for the add-drop filter. . . . . . . . . . . . . 30

3.3 Transmission and delay spectra for ADF, simulated using TMM . . . 33

3.4 Transmission and delay spectra for ADF, simulated using SMA . . . 37

3.5 Comparison of TMM and SMA simulation results. . . . . . . . . . . 39

4.1 An infinite 2D lattice of coupled ring resonators, without a magnetic field. . . . . . . . . . . . . . . . . 46

4.2 A plaquette of rings which implements magnetic field for photons. . . 51

4.3 An infinite 1D array of coupled rings, with hopping phase. . . . . . . 52

4.4 A $4 \times 4$ lattice of site rings, coupled to probe waveguides. . . . . . . . 55

4.5 Simulated transmission spectrum and edge states in a finite lattice. . 58

4.6 Transmission of a $8 \times 8$ lattice with weak input/output coupling. . . . 59

4.7 Flipping of the transmission spectrum as a result of spin reversal. . . 60

4.8 Simulated transmission and delay spectrum of a 20 ring array. . . . . 63

4.9 Effect of disorder in a $1 \mathrm{D}$ array. . . . . . . . . . . . . . . . 63

4.10 Transmission through edge states, in the presence of disorder. . . . . 64

4.11 A $2 \times 2$ lattice used to demonstrate the TMM for finite lattices. . . . . 66

4.12 Unfolded paths through the $2 \times 2$ lattice. . . . . . . . . . . . 66

4.13 Comparison of TMM and CMT simulation results. . . . . . . . . . 70 
4.14 Transmission spectrum for the link rings. . . . . . . . . . . . . 70

5.1 Cross-section of a typical silicon nanowaveguide. . . . . . . . . . . 73

5.2 A typical photolithography and etching step in the fabrication process. 74

5.3 SEM of a grating coupler used to couple light to the devices . . . . . 76

5.4 Experimental setup for transmission and IR intensity measurements. . 78

5.5 Experimental design and transmission spectrum of an ADF. . . . . . 80

5.6 Characterization of ADF using SMA . . . . . . . . . . . . . . 82

5.7 Comparison of a measured ADF spectrum with that simulated using FDTD ............................ 83

5.8 SEM image and observed spectra of a $8 \times 8$ lattice . . . . . . . . . . 84

5.9 Intensity seen by the CCD camera as a function of input frequency, for an $\mathrm{ADF} \ldots \ldots \ldots . \ldots . \ldots . \ldots . \ldots 87$

5.10 Experimental observation of edge states. . . . . . . . . . . . . . 88

5.11 Edge state routing around a lattice defect. . . . . . . . . . . . . . . 90

5.12 Measured and simulated spectra and delay distributions for $8 \times 8$ lattice devices. . . . . . . . . . . . . . . . . . . . . . . 91

5.13 Measured and simulated spectra and delay distributions for $15 \times 15$ lattice devices. . . . . . . . . . . . . . . . . . . . . . . . . . 94

5.14 Observed transmission and delay spectra for 10 ring 1D-array devices. 94

5.15 Transmission and delay-time scaling for 2D and 1D devices. . . . . . 96

5.16 Simulation results showing localization in a 1D array. . . . . . . . . 98

5.17 Reduced power coupling to the lattice in the short-edge region. . . . . 99

6.1 Square annulus geometry to measure the winding number of edge states. 108

6.2 Waveguide cross-section with heaters. . . . . . . . . . . . . . . . . 109

6.3 Shifting of eigenvalues in a square lattice. . . . . . . . . . . . . . . 110

6.4 Measured and simulated contour plots for the transmission spectra as a function of phase $\Phi$. . . . . . . . . . . . . . . . . . 111

6.5 A ring geometry to investigate winding number of topological, but not robust, states . . . . . . . . . . . . . . . . . . 113

6.6 Measured and simulated contour plots for the transmission spectra, for ring geometry. . . . . . . . . . . . . . . . . . . 114

6.7 Measured phase shift in an ADF, as a function of the heater power. . 116

7.1 Pump-probe setup to investigate the nonlinear effects. . . . . . . . . . 127

7.2 Measured change in refractive index and contrast as a function of ring intensity. . . . . . . . . . . . . . . . . . . 128

7.3 The problem of global shift. . . . . . . . . . . . . . . . 131

7.4 Differential shift of site and link ring resonance, in a three-ring device. 132

7.5 Measured transmission spectra in the presence of a strong pump. . . . 133

A.1 Spin degree of freedom in an ADF. . . . . . . . . . . . . . . 139

A.2 Effect of strong back-scattering on edge-state transmission. . . . . . . 140

B.1 Analysis of a three-ring device using TMM and CMT. . . . . . . . . 143 


\section{List of Abbreviations}

$\begin{array}{ll}\text { ADF } & \text { Add-Drop Filter } \\ \text { APF } & \text { All-Pass Filter } \\ \text { CMOS } & \text { Complementary Metal-Oxide-Semiconductor } \\ \text { CMT } & \text { Coupled-Mode Theory } \\ \text { FCA } & \text { Free Carrier Absorption } \\ \text { FCD } & \text { Free Carrier Dispersion } \\ \text { SEM } & \text { Scanning Electron Microscope } \\ \text { SMA } & \text { Single-Mode Approximation } \\ \text { SOI } & \text { Silicon-On-Insulator } \\ \text { TMM } & \text { Transfer Matrix Method } \\ \text { TPA } & \text { Two-Photon Absorption }\end{array}$




\section{Chapter 1: Introduction}

The presence of a magnetic field leads to fundamental changes in the transport properties of electronic systems. Classical examples are the Lorentz force and the Hall effect where magnetic field controls electron motion. The Lorentz force has been widely used in charged particle accelerators and magnetic lenses for electron beams, while the Hall effect has found substantial use in semiconductor material characterization. At the quantum level, the hallmark example of magnetic field affecting electronic motion is the quantum Hall effect [1-4]. In the quantum Hall effect, at low temperatures and high magnetic field, a two-dimensional (2D) electron gas exhibits quantized Hall conductivity. The underlying mechanism for this phenomena is the quantization of energy levels, called Landau levels, resulting from the application of a perpendicular magnetic field [5].

Further, electronic transport through a disordered two-dimensional system leads to localization where the conductivity falls exponentially as the system size increases [6]. However, for quantum Hall systems, the quantization of Hall conductivity is remarkably insensitive to disorder. This peculiar property of quantum Hall phenomena is due to the presence of robust and topologically protected edge states - states which are confined to the edge of the system $[7,8]$. Edge states are unidirec- 
tional, immune to back-scattering caused by disorder and hence are never localized. These states carry current and guarantee the quantization of conductance.

With the emergence of artificial photonic structures such as photonic crystals and metamaterials, there has been a significant interest in manipulating photonic transport using gauge fields, in a manner similar to electrons. Of particular importance is the need for one-way, reflection free waveguides which find applications in large scale photonic integration. Fabrication imperfections and environmental changes often lead to the undesirable back-reflection in ordinary waveguides and hence limit device functionalities. Implementing photonic analogs of chiral electronic edge states is, therefore, a natural choice to mitigate back-reflection and achieve robust waveguides.

In 2008, it was first proposed that analogs of the quantum Hall effect and edge states could be realized in photonic systems using the magneto-optic effect in gyro-magnetic photonic crystals $[9,10]$. Many proposals followed with specific implementations of the phenomena, and the presence of edge states was experimentally demonstrated first in the microwave regime $[11,12]$. However, subsequent proposals and attempts were limited to microwaves since the primary source of coupling - the magneto-optical effects - are very weak for optical photons $[13,14]$. In 2011, it was first realized that photonic analogs of quantum Hall edge states could be implemented by engineering a synthetic magnetic field for photons [15]. The proposed system was a 2D lattice of coupled microring resonators which would mimic the tight-binding Hamiltonian with a magnetic field $[8,16]$. The resonators act as lattice sites where photons are bound, but, with some probability to tunnel to neigh- 
bouring resonators. To introduce the synthetic magnetic field, the resonators were arranged such that a round trip along any plaquette (consisting of a $2 \times 2$ array of resonators) results in a total accumulated phase of $\phi$ - the equivalent of magnetic flux. It was theoretically shown that the edge states are topologically robust against lattice disorders. This system, however, does not explicitly break the time reversal symmetry as do the quantum Hall systems with real magnetic field and hence, is not immune to all types of fabrication disorders, e.g. back-scattering.

Following Ref. [15], there have been a number of remarkable proposals and experimental efforts to implement synthetic gauge field for photons. In Ref. $[17,18]$, imitating graphene, a system of coupled 1D waveguides has been implemented where a non-uniform strain applied to the lattice results in a pseudo-magnetic field. A similar system has been reported in Ref. [19] where helical waveguides were used to demonstrate the presence of edge states. Another proposal makes use of the dynamic modulation of the coupling between lattice sites to explicitly break the time-reversal symmetry $[20,21]$ and implement magnetic field in real space. This approach has been demonstrated using radio waves [22]. Photonic edge states have also been studied extensively using metamaterials $[13,14]$.

This thesis reports the experimental implementation of a synthetic magnetic field for photons and our observation of the topologically robust photonic edge states, employing the 2D coupled ring resonator system studied in Ref. [15] (Fig. 1.1). This system has the particular advantage of being directly adaptable to photonic integrated circuits because it has been implemented in a versatile silicon-on-insulator platform using CMOS-compatible fabrication process. In this system, we show the 


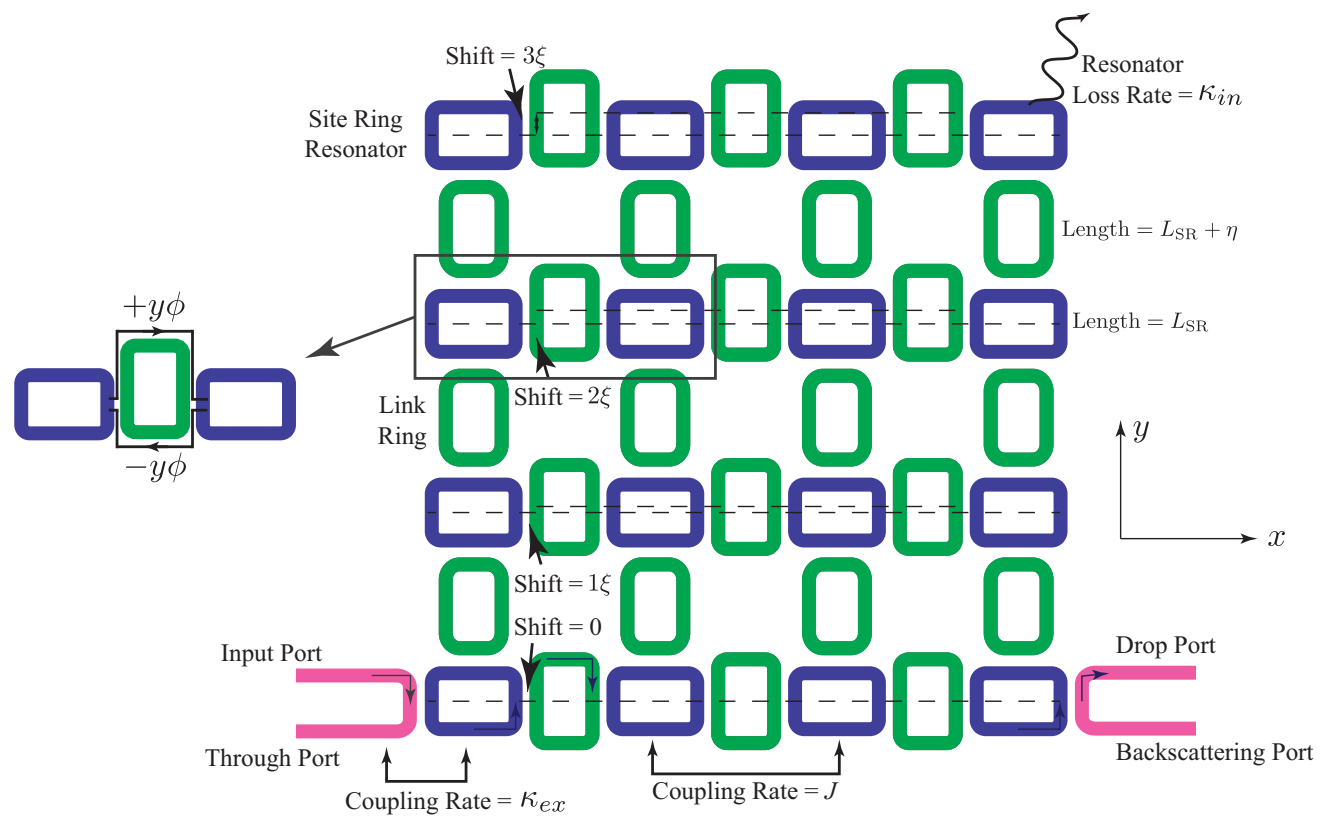

Figure 1.1: The 2D lattice of coupled ring resonators used to implement a synthetic gauge field for photons. The main site rings (blue) are coupled using another set of link rings (green). The link rings that are placed horizontally between site rings are shifted in a particular fashion so as to introduce a uniform magnetic field throughout the lattice. The lattice is coupled to input and output waveguides (red) to probe the transmission and delay spectrum of the device. 
presence of topological edge states by directly imaging the path followed by light in the lattice. The edge states route around a deliberately induced lattice defect and do not scatter into the bulk of the lattice. We report the first quantitative analysis of the robustness of edge states using transport properties (transmission and delay). Using delay-time distributions, we demonstrate that the edge states avoid localization induced by fabrication imperfections [23-26].

Topological edge states are characterized by their winding number, a topologically invariant integer $[8,27-29]$. We measure the winding number of edge states in our system and show that in the photonic analog of the quantum Hall effect, it is the winding number that is quantized, analogous to quantization of Hall conductivity in electronic systems. Furthermore, the use of ring resonators greatly enhances nonlinear effects in silicon, which we use to explore the effect of nonlinear interactions on the edge states.

In Chapter 2, we begin with a discussion of the basics of quantum Hall effect. We study the tight-binding model for quantum Hall effect and the emergence of topological edge states. In Chapter 3, we discuss a ring resonator - the basic building block of our system. Chapter 4 describes our implementation of synthetic magnetic field and photonic edge states, using a 2D lattice of coupled ring resonators.

Chapter 5 details the experimental implementation of our system on a silicon photonics platform and the observation of robust edge states. Chapter 6 reports the winding number measurements for the edge states. Finally, in Chapter 7, we investigate the effect of nonlinear interactions in silicon ring resonators on edge states, using a pump-probe technique. 


\section{Chapter 2: The Quantum Hall Effect}

In 1980, while working with two-dimensional electron gas (2DEG) confined in silicon field effect transistors, at low temperatures and high magnetic field, von Klitzing et.al. found that when the gate voltage is varied, the Hall conductivity of the sample exhibits plateau regions [1]. The Hall conductivity value at the plateau regions was found to be quantized as

$$
\sigma_{\mathrm{H}}=n \frac{e^{2}}{h}
$$

where $n$ is an integer. Along with this, at the plateau regions of Hall conductivity, the longitudinal conductivity was negligible (Fig. 2.1). Soon, it was found that this phenomena is not particular to von Klitzing's system and it was demonstrated in other systems as well, for example in 2D electron gas confined at the interface of a GaAs-AlGaAs heterostructure [2,4]. More importantly, the quantization of Hall conductivity was remarkably precise, to 1 part in $10^{7}$, independent of the device geometry, material properties, impurities, etc.

The quantum Hall effect is a direct manifestation of the quantization of energy levels, called Landau levels, in a 2D electron gas with an external magnetic field applied perpendicular to the $2 \mathrm{D}$ plane $[1,7,30,31]$. The presence of Landau levels explains the quantization of the Hall conductance and the vanishing of lon- 

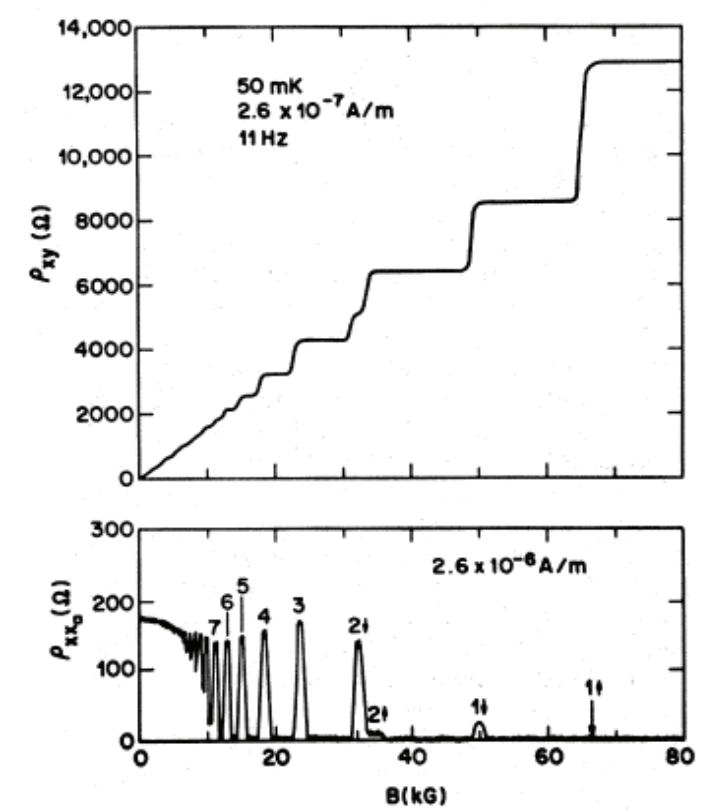

Figure 2.1: Observed Hall $\left(\rho_{x y}\right)$ and Longitudinal resistivity $\rho_{x x}$, as a function of applied magnetic field [4]. Plateau regions in Hall resistivity coincide with troughs in longitudinal resistivity. The markings indicate number of filled Landau levels. As the magnetic field increases, the number of filled Landau levels below the Fermi energy decreases and hence the resistivity increases.

gitudinal conductance. But the insensitivity of quantization to system properties can only be explained by invoking gauge invariance $[7,31]$ and topological considerations $[27,28,32]$. It has been shown that the quantization of Hall conductance is a result of the gauge invariance associated with the magnetic vector potential, and since gauge invariance is independent of material properties, so is the quantization. Furthermore, disorder in the system leads to localization of electron states that occupy the bulk of the system and hence, they do not carry current $[6,7,33,34]$. The current is carried only by topologically ordered, chiral edge states - the states confined to the perimeter of the system $[7,8,35]$. Edge states are immune against disorder induced localization and therefore guarantee the quantization of conductance. 


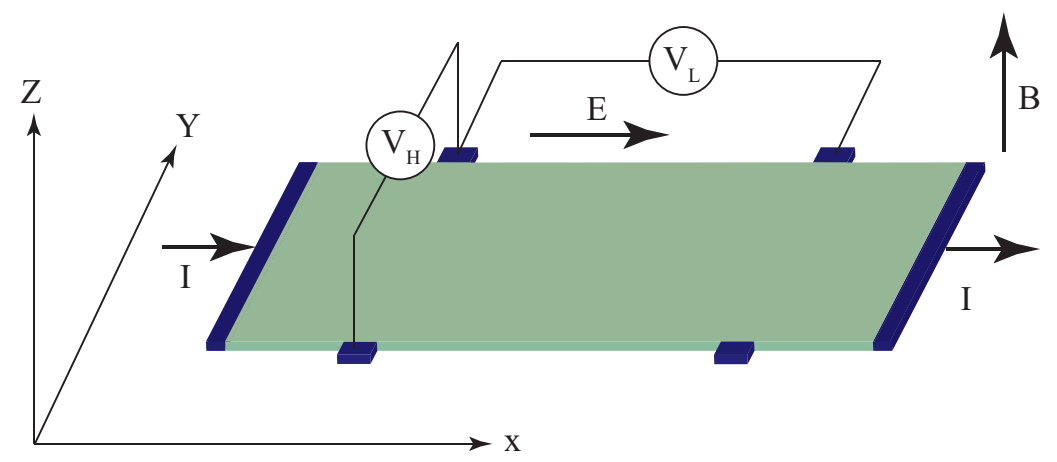

Figure 2.2: Hall Geometry. Applied electric field is along $x$-axis and the Hall voltage $\left(V_{\mathrm{H}}\right)$ develops across $y$-axis.

Subsequent experiments also found plateaus at fractional multiples (such as $1 / 3,2 / 3$ ) of $\frac{e^{2}}{h}$ - a phenomena termed as the Fractional Quantum Hall effect [3, 36]. To explain the fractional values of quantization, it is necessary to include the electron-electron interactions in the system, which are not considered in the Integer Quantum Hall effect (IQHE). In this report, however, we use only the integer quantum Hall effect. In the following sections, we briefly review the quantization of energy levels and the Hall conductance. We discuss the tight-binding model used to study quantum-Hall effect and explore the topological aspects of edge states.

\subsection{The Classical Hall Effect}

In the classical Hall effect, when a thin sheet of conductor is placed in the presence of perpendicular electric and magnetic fields, with the magnetic field being threading the conductor surface, a current called the Hall current flows in a direction perpendicular to the applied fields (Fig. 2.2). The Hall current $\left(I_{\mathrm{H}}\right)$ and the applied 


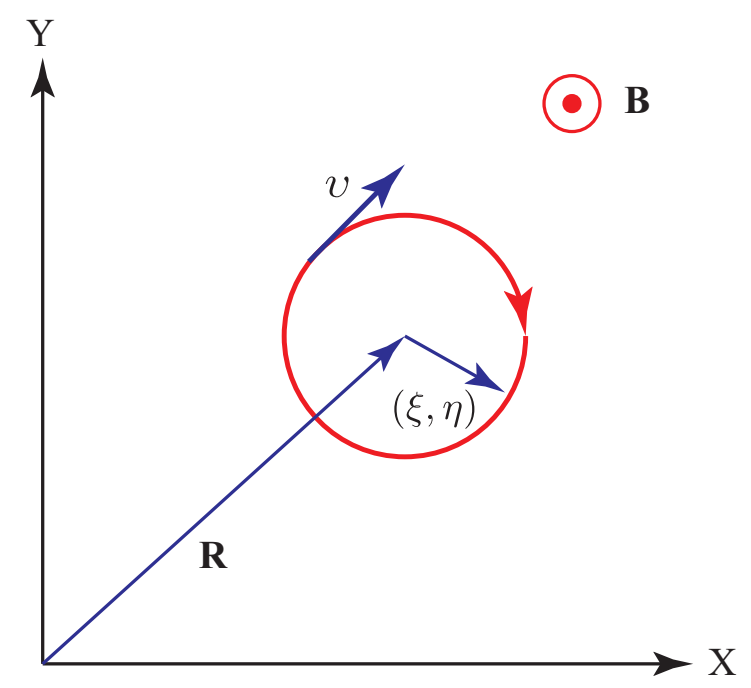

Figure 2.3: Cyclotron motion in a perpendicular magnetic field. The coordinates $(\mathbf{R})$ of the center are arbitrary.

electric field $(\mathcal{E})$ are related through Hall conductivity $\sigma_{\mathrm{H}}$ as $[37,38]$

$$
I_{\mathrm{H}}=\sigma_{\mathrm{H}} \mathcal{E}
$$

The Hall conductivity is found to decrease monotonically with increasing magnetic field. To understand the classical Hall effect and Hall conductivity, we first look at the classical motion of an electron in a two-dimensional plane in the presence of a magnetic field and then in the presence of both the electric and magnetic fields.

\subsubsection{Electron Motion in a Magnetic Field}

The classical electron motion under the influence of a magnetic field is governed by the equation

$$
m_{\mathrm{e}} \frac{d^{2} \boldsymbol{r}}{d t^{2}}=e \boldsymbol{v} \times \boldsymbol{B}
$$

where $\boldsymbol{r}$ is the position coordinate and $\boldsymbol{v}=\frac{d \boldsymbol{r}}{d t}$ is the electron velocity (Fig.2.3). Electrons perform cyclotron motion with frequency $\omega_{c}=\frac{e B}{m_{\mathrm{e}}}$ and radius $r_{0}=\frac{m_{\mathrm{e}} v}{e B}$ 
around an arbitrary center with position coordinates $\boldsymbol{R}=(X, Y)$ determined by initial conditions, that is,

$$
\begin{aligned}
\boldsymbol{r} & =\boldsymbol{R}+r_{0}\left(\cos \left(\omega_{c} t\right), \sin \left(\omega_{c} t\right)\right) \\
\boldsymbol{v} & =r_{0} \omega_{c}\left(-\sin \left(\omega_{c} t\right), \cos \left(\omega_{c} t\right)\right) .
\end{aligned}
$$

The position and velocity vectors can further be written as

$$
\begin{aligned}
\boldsymbol{r} & =(X+\xi, Y+\eta) \\
\boldsymbol{v} & =\omega_{c}(-\eta, \xi) .
\end{aligned}
$$

We will use these relations and the arbitrariness of guiding center coordinates $(X, Y)$ when analysing the quantum mechanics of electrons in the presence of a magnetic field.

\subsubsection{Electron Motion in Electric and Magnetic Fields}

In the presence of perpendicular electric and magnetic fields, the classical equation for motion of an electron is

$$
m_{\mathrm{e}} \frac{d^{2} \boldsymbol{r}}{d t^{2}}=e(\boldsymbol{E}+\boldsymbol{v} \times \boldsymbol{B}) .
$$

The solution to this equation yields a superposition of drift motion and cyclotron motion, with the direction of drift being perpendicular both to the electric and magnetic fields. The electron velocity vector is now given as [38]

$$
\boldsymbol{v}=\left(-r_{0} \omega_{c} \sin \left(\omega_{c} t\right), r_{0} \cos \left(\omega_{c} t\right)+v_{0}\right)
$$


where $v_{0}$ is the drift component given as

$$
\boldsymbol{v}_{\mathbf{0}}=\frac{\boldsymbol{E} \times \boldsymbol{B}}{B^{2}}
$$

For an applied magnetic field along $z$-axis and electric field along $x$-axis, the drift motion is along $y$-axis. This drift component of electron motion generates the Hall effect. The current density generated along the $y$-axis is then

$$
j=n e v_{0}=-\frac{n e E}{B}=\sigma_{\mathrm{H}} E
$$

This is precisely the Hall current $I_{\mathrm{H}}$ and the Hall conductivity is therefore given as

$$
\sigma_{\mathrm{H}}=-\frac{n e}{B}
$$

The Hall conductivity $\sigma_{\mathrm{H}}$ thus decreases monotonically as the magnetic field increases.

\subsection{The Quantum Hall Effect}

In the quantum Hall effect, which is seen at very low temperature and high magnetic field, the Hall conductivity shows quantized plateau regions with increasing magnetic field, contrary to what is expected classically. To understand this quantization of Hall conductivity and the quantum Hall effect, we discuss the quantum mechanics of electrons first in the presence of a magnetic field and then in the presence of both electric and magnetic fields. 


\subsubsection{Electron Motion in a Magnetic Field}

In the presence of a magnetic field, the Hamiltonian for a 2DEG can be written

as

$$
H=\frac{1}{2 m_{\mathrm{e}}}(\boldsymbol{p}-e \boldsymbol{A}(\boldsymbol{r}))^{2}=\frac{1}{2 m_{\mathrm{e}}} \boldsymbol{\pi}^{2}
$$

where $\boldsymbol{p}$ is the canonical momentum operator and $\boldsymbol{A}(\boldsymbol{r})$ is the vector potential resulting from the applied magnetic field. The dynamical momentum operator $\boldsymbol{\pi}$ is related to the velocity vector $\boldsymbol{v}$ and can be expressed as

$$
\boldsymbol{\pi}=m_{\mathrm{e}} \boldsymbol{v}=m_{\mathrm{e}} \frac{i}{\hbar}[H, r]=\boldsymbol{p}-e \boldsymbol{A} .
$$

Further, the components of the dynamical momentum operator follow the commutation relation

$$
\left[\pi_{x}, \pi_{y}\right]=i \hbar e B=-i \frac{\hbar^{2}}{l_{0}^{2}},
$$

where $l_{0}$ is the magnetic length defined as

$$
l_{0}^{2}=\frac{\hbar}{e B}
$$

Using the above commutation relation, the Hamiltonian in (2.13) can be cast in the simple harmonic oscillator form

$$
H=\hbar \omega_{c}\left(a^{\dagger} a+\frac{1}{2}\right)
$$

where the creation and annihilation operators are

$$
\begin{aligned}
a & =\frac{1}{\sqrt{2} \hbar}\left(\pi_{x}-\pi_{y}\right) \\
a^{\dagger} & =\frac{1}{\sqrt{2} \hbar}\left(\pi_{x}+i \pi_{y}\right)
\end{aligned}
$$


and $\omega_{c}=\frac{e B}{m_{\mathrm{e}}}$ is the classical cyclotron frequency. Thus, we see that the energy eigenvalues of the Hamiltonian are quantized as

$$
E_{n}=\hbar \omega_{c}\left(n+\frac{1}{2}\right)
$$

It is these quantized, equally spaced energy levels which are called the Landau levels.

Now, following classical analysis of the motion of an electron in a magnetic field, we explore the freedom associated with the guiding center of the cyclotron motion. Using Eqs. 2.6-2.7, the position operator for the electrons is given as [38]

$$
\boldsymbol{r}=\left(X+\frac{l_{0}^{2}}{\hbar} \pi_{y}, Y-\frac{l_{0}^{2}}{\hbar} \pi_{x}\right)
$$

where $X, Y$ are the operators corresponding to the guiding center of cyclotron motion. Further, using the commutation relation for components of the position operator $\boldsymbol{r}$, we find that the operators $X, Y$ commute with the Hamiltonian but do not commute with each other i.e.

$$
[X, Y]=i l_{0}^{2}
$$

Since $X, Y$ commute with the Hamiltonian, the Landau levels are degenerate with respect to $X, Y$. To address this degeneracy, we label the eigenstates using the angular momentum operator

$$
L_{z}=(\boldsymbol{r} \times \boldsymbol{p})_{z}=-\frac{\hbar^{2}}{2 l_{0}^{2}}\left(X^{2}+Y^{2}\right)+\frac{l_{0}^{2}}{2 \hbar}\left(\pi_{x}^{2}+\pi_{y}^{2}\right)
$$

Both the terms in the above expression are of the harmonic oscillator form and therefore can be written as

$$
L_{z}=\hbar\left(a^{\dagger} a-b^{\dagger} b\right),
$$


where

$$
\begin{aligned}
b & =\frac{1}{\sqrt{2 l}}(X+i Y) \\
b^{\dagger} & =\frac{1}{\sqrt{2 l}}(X-i Y) .
\end{aligned}
$$

The eigenvalues of $L_{z}$ are integer numbers and can be used to label the degenerate eigenstates of the Hamiltonian. We label the eigenstates as $|n, m\rangle$, where the first index $n$ represents the energy eigenvalue (the Landau level) and $n-m$ is the eigenvalue of the operator $L_{z}$. Using $a|0,0\rangle=0$ and $b|0,0\rangle=0$ and the position representations of operators $a, b$, the ground state eigenfunction $|0, m\rangle$ in the coordinate representation is [38]

$$
\psi_{0, m}(\boldsymbol{r})=\frac{1}{\sqrt{2 \pi 2^{m} m ! l}}\left(\frac{x-i y}{l}\right)^{m} e^{-\frac{r^{2}}{4 l^{2}}}
$$

This wavefunction represents a electron density cloud confined to the perimeter of a circle with radius $\sqrt{2 m} l_{0}$. This is not the radius for cyclotron motion which is given by $l_{0}$. This state $|0, m\rangle$ represents many cyclotron orbits with their guiding center positioned on the bigger circle of radius $\sqrt{2 m} l_{0}$ (Fig. 2.4). The degeneracy in the eigenvalues of the angular momentum thus corresponds to the arbitrariness of the guiding center of the cyclotron motion. We will use this phenomena to show the robustness of edge states using gauge invariance and also to measure the winding number, an integer characterizing topological order of the edge states.

Moreover, if the area of the 2D electron gas is $S$ and since the area occupied by each state is $2 \pi l_{0}^{2}$ (using Eq. 2.22), the maximum value of $m$ and hence the 


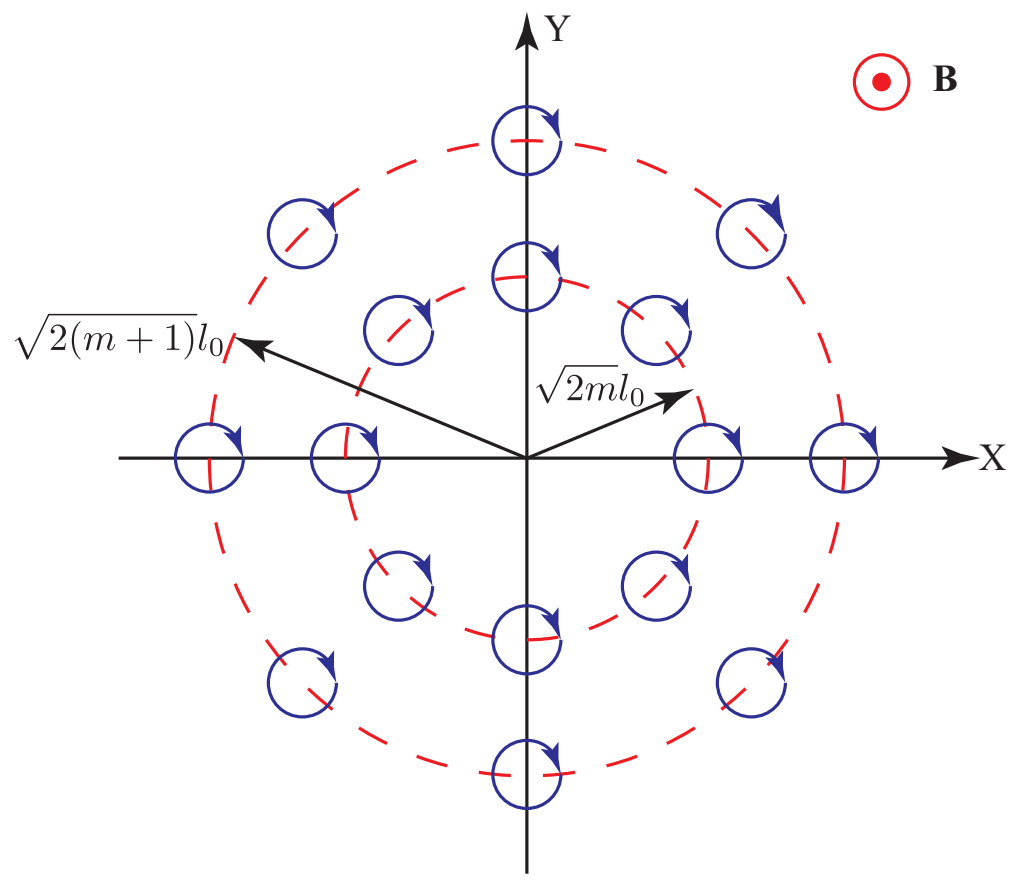

Figure 2.4: Classical cyclotron motion of electron superimposed over quantized wavefunction in Eq. 2.27. The guiding center of cyclotron motion is confined to the perimeter of a circle with radius $\sqrt{2 m} l_{0}$.

degeneracy in the Landau levels is

$$
m_{\max }=\frac{S}{2 \pi l_{0}^{2}}
$$

Incidentally, the magnetic flux penetrating this unit area is

$$
\Phi_{0}=B 2 \pi l_{0}^{2}=\frac{h}{e}
$$

which is the flux quantum. Therefore, the degeneracy in each Landau level is the number of flux quanta threading the system.

Next, we analyze the translation operators of the system. Since the magnetic field is uniform, the system is translation invariant. Therefore neither the canonical momentum $\boldsymbol{p}$, nor the dynamic momentum operator $\boldsymbol{\pi}$ are the generator of translation because they does not commute with the Hamiltonian. We can find another 
operator called pseudo-momentum operator defined as

$$
\Pi=\boldsymbol{p}-e \boldsymbol{A}+e \boldsymbol{B} \times \boldsymbol{r}
$$

which commutes with the Hamiltonian and is the generator of translation. Using classical considerations, the pseudo-momentum operator can in fact be related to the coordinates $(X, Y)$ of the guiding center of cyclotron motion of the electrons as

$$
\begin{aligned}
& \Pi_{x}=-e B Y \\
& \Pi_{y}=e B X
\end{aligned}
$$

The translation operator $\hat{\boldsymbol{T}}(\delta)=e^{i \delta \boldsymbol{\Pi}}$ is then called the magnetic translation operator. Since the components of pseudo-momentum operator do not commute

$$
\left[\Pi_{x}, \Pi_{y}\right]=-i \hbar e B
$$

the magnetic translation operators also do not commute, i.e.,

$$
\hat{T}_{x}\left(\Lambda_{x}\right) \hat{T}_{y}\left(\Lambda_{y}\right)=e^{2 \pi i \phi} \hat{T}_{y}\left(\Lambda_{y}\right) \hat{T}_{x}\left(\Lambda_{x}\right) .
$$

Translation in a closed path thus generates a phase $\phi=\frac{\Lambda_{x} \Lambda_{y}}{l_{0}^{2}}$, i.e.

$$
\hat{T}_{x}^{-1}\left(\Lambda_{x}\right) \hat{T}_{y}^{-1}\left(\Lambda_{y}\right) \hat{T}_{x}\left(\Lambda_{x}\right) \hat{T}_{y}\left(\Lambda_{y}\right)=e^{2 \pi i \phi} .
$$

The phase $\phi$ here is the induced Aharanov-Bohm phase. Thus, we see that the effect of applying a magnetic field is essentially the introduction of a non-zero phase for translation in a closed path. This crucial fact will be used when implementing a system which simulates magnetic field for photons. 


\subsubsection{Electron Motion in Electric and Magnetic Fields}

We now discuss the motion of electrons when both the electric and magnetic fields are applied, and derive an expression for the quantized Hall conductivity. We consider a system of length $L$ where an external electric field is applied along $x$-axis. The Hamiltonian for this system is

$$
H=\frac{1}{2 m_{e}} \pi^{2}-e \mathcal{E} x
$$

If we choose the Landau gauge, i.e., $\boldsymbol{A}=(0, B x, 0)$, the Hamiltonian commutes with $p_{y}$ and therefore the eigenfunctions of the Hamiltonian can be written as

$$
\psi(x, y)=\frac{1}{\sqrt{L}} \exp \left(-i k_{y} y\right) \varphi(x)
$$

Using this in (2.36) yields

$$
\left(\frac{1}{2 m_{e}} p_{x}^{2}+\frac{m_{e} \omega_{c}^{2}}{2}(x-X)^{2}\right) \varphi(x)=\left[E_{X}+e E X-\frac{m_{e}}{2}\left(\frac{E}{B}\right)^{2}\right] \varphi(x)
$$

The left hand side of this equation is the Hamiltonian without the electric field, but now shifted along $x$-axis. The energy of the state is therefore,

$$
E_{X}=\left(n+\frac{1}{2}\right) \hbar \omega_{c}-e E X+\frac{m_{e}}{2}\left(\frac{E}{B}\right)^{2}
$$

The first term here is the usual Landau level energy. The second term is the electrostatic potential energy because of the applied electric field. This electrostatic energy depends on the position $X$ of the guiding center and hence states partially lifts the degeneracy associated with the $X$ coordinate. States with different $Y$ coordinates are still degenerate. 
The last term represents the kinetic energy associated with a drift of the electrons along $y$-axis, with a velocity of magnitude $\left(\frac{E}{B}\right)$, calculated as

$$
\left\langle\varphi\left|v_{y}\right| \varphi\right\rangle=\left\langle\varphi\left|\frac{1}{m_{e}}\left(p_{y}-e B x\right)\right| \varphi\right\rangle=-\frac{E}{B}
$$

Moreover, the electrons do not travel along the $\mathrm{x}$ axis, since

$$
\left\langle\varphi\left|v_{x}\right| \varphi\right\rangle=\left\langle\varphi\left|\frac{p_{x}}{m_{e}}\right| \varphi\right\rangle=0
$$

Thus, the electrons drift along equipotential lines in a direction perpendicular to the applied electric field and hence generate the Hall current. In classical sense, the trajectory of motion is thus in fact a cycloid, with a drift imposed on the cyclotron rotation motion. For a 2D system with unit area, with $n-1$ filled Landau levels, that is, when the fermi energy $E_{\mathrm{F}}$ is in the gap between $n-1$ and $n$ Landau levels, the total number of electrons $n_{e}$ that contribute to Hall current, including the degeneracy in $(Y)$ coordinate of the guiding center, is

$$
n_{e}=n \frac{1}{2 \pi l_{0}^{2}}
$$

Here we have used the fact that the number of degenerate states is same for all Landau levels. The total Hall current is

$$
I_{y}=e n_{e} v_{y}=\frac{n e^{2}}{h} \mathcal{E}
$$

and the Hall conductivity is therefore,

$$
\sigma_{\mathrm{H}}=\frac{I_{y}}{\mathcal{E}}=\frac{n e^{2}}{h}
$$

Furthermore, since the expectation value of $v_{x}$ is zero, the longitudinal (or diagonal) conductivity (and also the longitudinal resistivity) is zero. Thus we see that for a $2 \mathrm{D}$ 

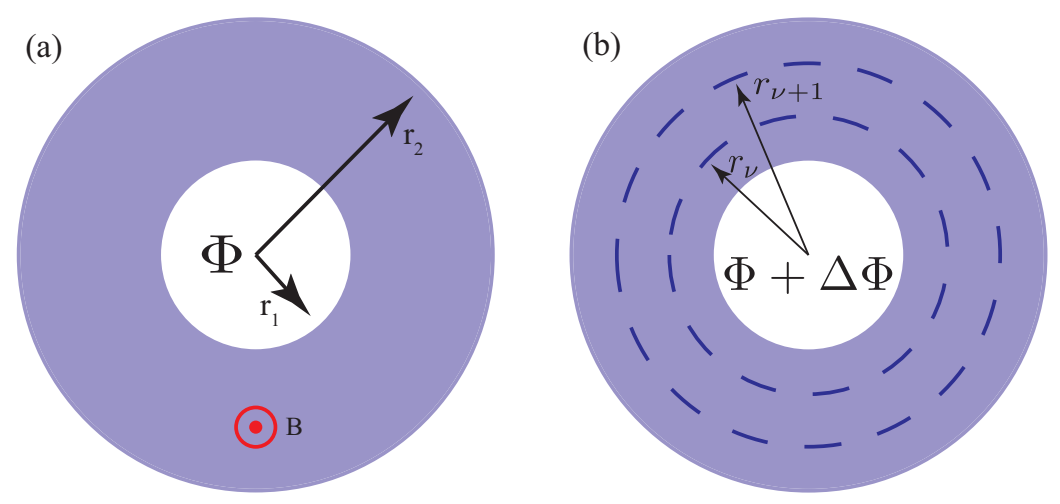

Figure 2.5: The Corbino geometry for studying the Hall effect [7]. (a) An annulus with a uniform magnetic field $\boldsymbol{B}$ and a variable magnetic flux $\Phi$ threading the center hole. (b) As the magnetic flux in the hole increases, the locus of the guiding center for classical cyclotron motion expands in radius.

electron gas in the presence of a magnetic field, the Hall conductivity is quantized. Whenever the Fermi level $E_{\mathrm{F}}$ is in the middle of two Landau levels, the exact position of the Fermi level does not change the number of electrons that contribute to conduction and hence the Hall conductivity shows plateau regions, accompanied by vanishing longitudinal conductivity. Whenever, $E_{\mathrm{F}}$ passes through a Landau level, the electrons in that Landau level contribute to conduction and the Hall conductivity jumps to the next quantized level.

\subsection{Robustness of Quantization and Gauge Invariance}

So far, we have only shown that the Hall conductivity is quantized. It still remains to show that the quantization is robust against material impurities. Laughlin proposed that the quantization of Hall conductance is in fact a consequence of gauge invariance and is therefore immune against disorder [31]. To show this gauge invariance, we consider the Corbino geometry (Fig. 2.5) - a 2D electron gas confined 
in a annular region, with a uniform magnetic field $B$ perpendicular to the plane [7]. This geometry has the advantage that the hole of the annulus can be threaded by a variable magnetic flux $\Phi$. We choose a gauge such that the vector potential is given as

$$
A(r)=\frac{1}{2} B r+\frac{\Phi}{2 \pi r}
$$

where the second term is the contribution to vector potential by magnetic flux in the hole. When the flux in the interior region is changed by $\delta \Phi$, the vector potential undergoes a gauge transformation. Under the effect of this gauge transformation, the wavefunction acquires a phase factor as seen

$$
\psi^{\prime}=\psi e^{i \frac{e}{\hbar} \delta \Phi}=\psi e^{2 \pi i \frac{\delta \Phi}{\Phi_{0}}} .
$$

But since the electron states extend throughout the circumference of the ring, the wavefunction is single-valued which forces $\frac{\delta \Phi}{\Phi_{0}}$ to be an integer. Each unit quanta increase in flux is equivalent to an increase in the angular momentum eigenvalue by unity (see Eqs. 2.24-2.27). The locus of the guiding center for each of these extended states (including different Landau levels) therefore moves towards the outer edge of the ring and occupies the position once occupied by its neighboring degenerate state. The system thus returns exactly to its previous state, where the position of a state is now occupied by its preceding state. But in this process, $n$ electrons are transferred from the inner edge to the outer edge (for $n$ filled Landau levels). If the potential difference between the inner and outer edges is $V$, the energy cost of moving these electrons is $\Delta E=-n e V$. Now, the induced Hall current in the ring can be written 


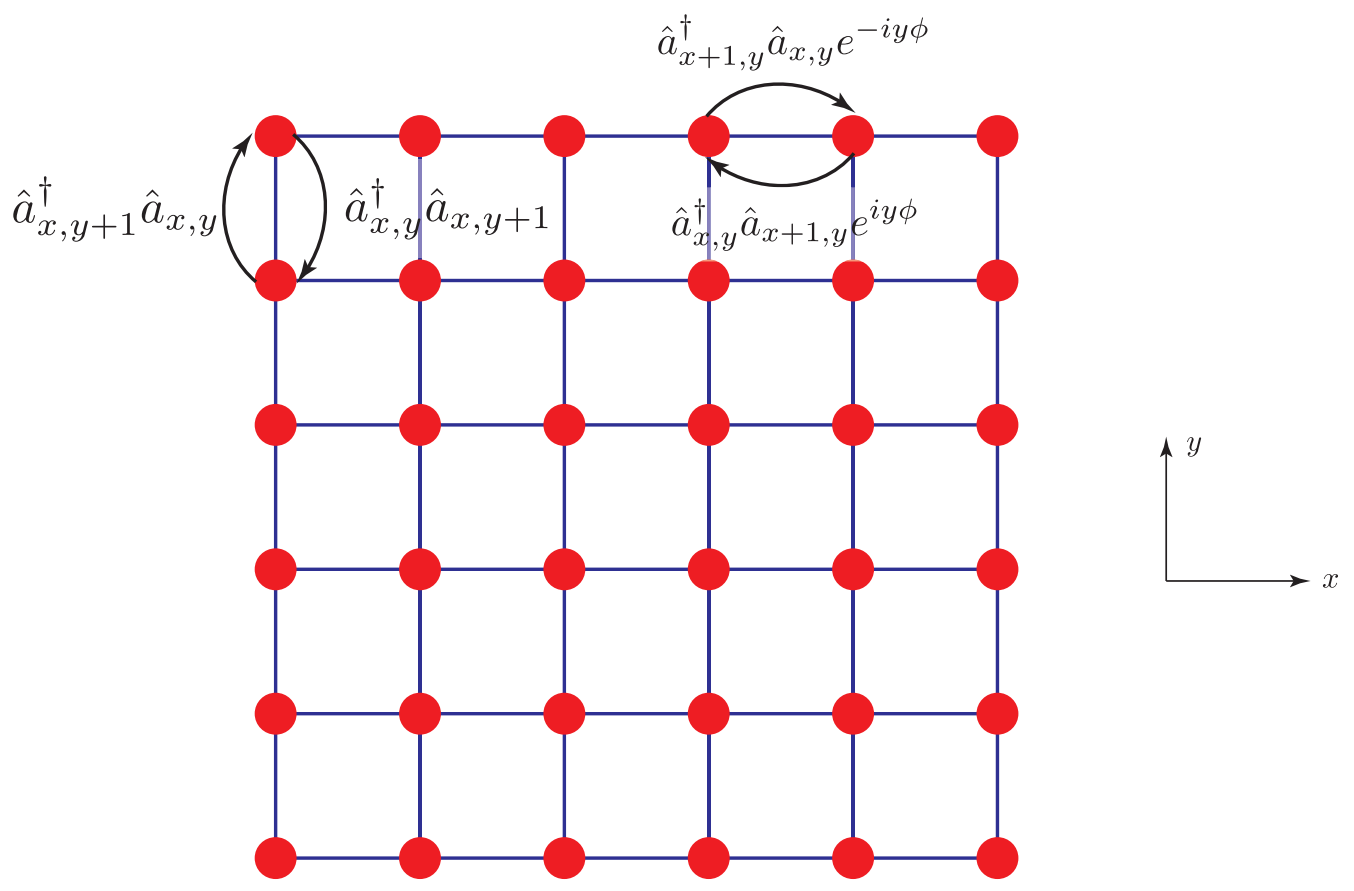

Figure 2.6: A 2D square lattice used to study the 2DEG. The electrons are bound to lattice sites (red) which represent a periodic crystal potential. The electrons can tunnel to their nearest neighbors with a tunneling rate $J$. Depending on the gauge used, hopping along $x$-axis or $y$-axis can have a direction-dependent phase. Here we chose the Landau gauge.

as $[31]$

$$
I=\frac{\Delta E}{\Delta \Phi}=\frac{n e V}{\Phi_{0}}=\frac{n e^{2}}{h} V .
$$

The Hall conductivity is therefore $\sigma_{\mathrm{H}}=\frac{n e^{2}}{h}$. Using these arguments, we can see that the accurate quantization of Hall conductivity is a result of gauge invariance of the Hamiltonian with respect to a vector potential $\boldsymbol{A}$. In Chapter 6, we will show that this phenomena of shifting of states from inner to outer edge can be used to measure the winding number of edge states in our photonic system. 


\subsection{The Tight-Binding Model}

The Hamiltonian in Eq. 2.13 for a continuum system of 2DEG in a magnetic field can be reformulated by discretizing the Hamiltonian on a square lattice [8, 39,40]. Physically, the effect of lattice sites is equivalent to a periodic potential in the crystal to which the electrons are bound. Also, the electrons have a finite probability to tunnel to other lattice sites. Here we consider only the tight-binding model where the electrons can tunnel only to their nearest neighbors (Fig.2.6). In the limit $\Lambda \ll l_{0}$, where $\Lambda$ is the lattice spacing, one recovers the continuum theory. To discretize the Hamiltonian,

$$
H=\frac{1}{2 m_{e}}(\boldsymbol{p}-e \boldsymbol{A})^{2}+V
$$

where $V$ is the periodic potential, we approximate the momentum operator acting on wavefunction $\psi(x, y)$ in Eq. 2.13 as

$$
\boldsymbol{p} \psi(x, y)=-i \hbar \nabla \psi(x, y)=-i \hbar\left[\frac{\psi(x+\Lambda, y)-\psi(x, y)}{\Lambda}+\frac{\psi(x, y+\Lambda)-\psi(x, y)}{\Lambda}\right]
$$

The Hamiltonian in this model (choosing Landau gauge for magnetic field, $A=$ $(0, B x, 0))$ can then be written as $[16]$

$$
H_{0}=\sum_{x, y} \hat{a}_{x, y}^{\dagger} \hat{a}_{x, y}-J\left(\sum_{x, y} \hat{a}_{x+1, y}^{\dagger} \hat{a}_{x, y} e^{-i y \phi}+\hat{a}_{x, y}^{\dagger} \hat{a}_{x+1, y} e^{i y \phi}+\hat{a}_{x, y+1}^{\dagger} \hat{a}_{x, y}+\hat{a}_{x, y}^{\dagger} \hat{a}_{x, y+1}\right) .
$$

The first term here arises from the periodic potential $V$. The second and third terms represent tunneling along $\pm x$-axis, with a tunneling rate $J$, and with a direction and 


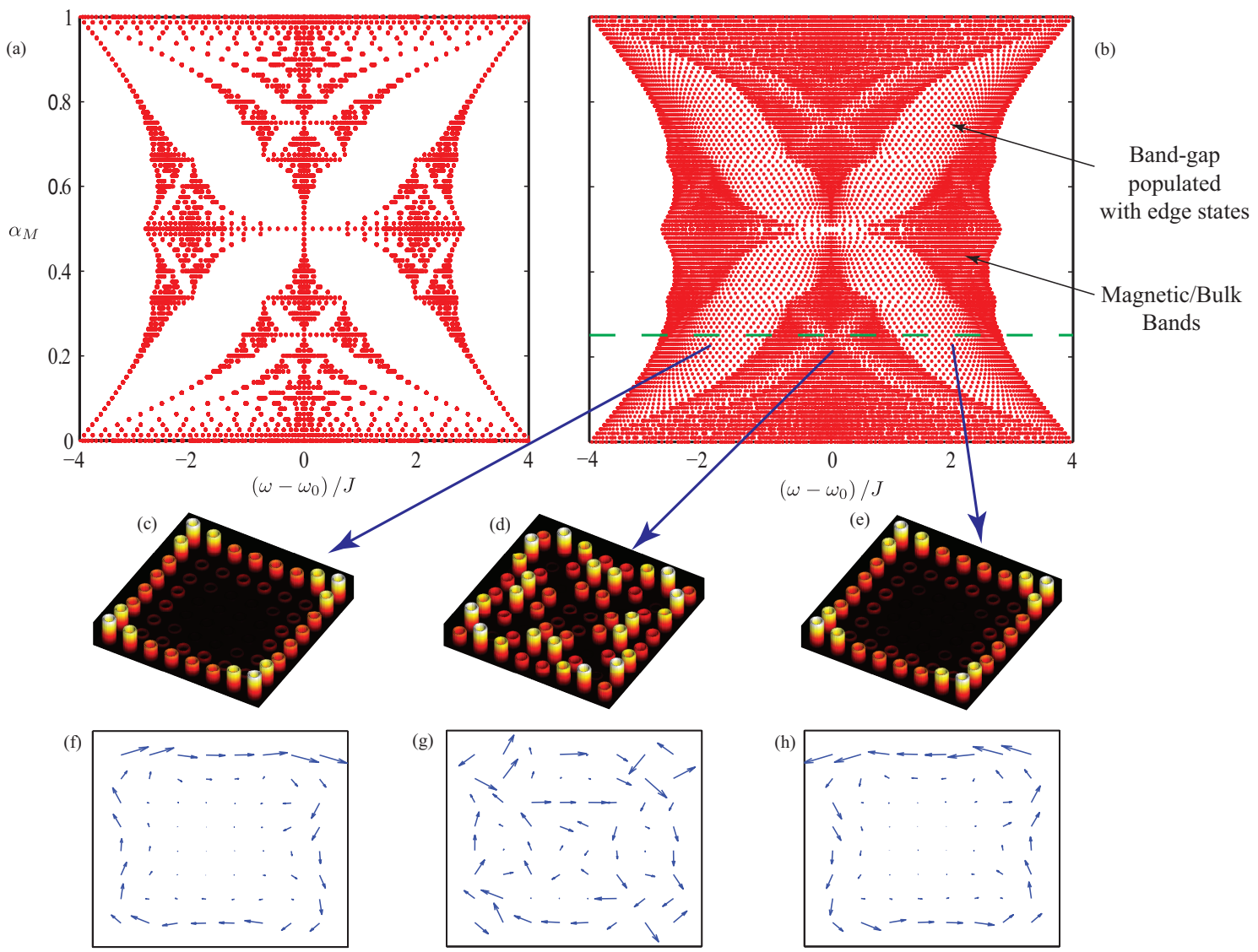

Figure 2.7: (a) Hofstadter energy spectrum for a lattice with periodic boundary conditions. (b) Energy spectrum for a finite lattice. The bandgaps are now populated with edge states. (c-e) Simulated intensity plots for edge and magnetic bands eigenfunctions, for $\alpha_{\mathrm{M}}=\frac{1}{4}$. Edge state wavefunctions are confined to the perimeter of the lattice, whereas the bulk eigenfunctions are spread throughput the lattice. (eg) Corresponding probability currents. Edge states on the left and right bandgaps travel in opposite directions.

position-dependent phase $y \phi$. The last two terms represent hopping along $\pm y$-axis, without any hopping phase (since we chose the Landau gauge).

\subsubsection{Hofstadter Spectrum and Edge States}

For an infinite lattice, the energy eigenvalues of the tight-binding Hamiltonian group into allowed energy bands separated by bandgaps $[8,16,39]$. When the energy eigenvalues are plotted against magnetic field strength $\alpha_{\mathrm{M}}$, the famous Hofstadter 
Butterfly spectrum emerges as shown in Fig. 2.7(a) [39]. For $\alpha_{\mathrm{M}}=\frac{p}{q}$, where $p$ and $q$ are co-prime integers, the spectrum has $q$ allowed bands (called bulk bands), interleaved with $q-1$ bandgaps. Also, when $q$ is even, the centermost bands are degenerate at $\omega=0$, i.e., the two bands touch each other.

For a finite lattice, the otherwise forbidden band-gaps are populated by so called edge-states. Fig. 2.7(b) shows the Hofstadter spectrum for a finite lattice. For $\alpha_{\mathrm{M}}=\frac{1}{4}$, there are 4 allowed bands with 3 band-gap regions. To highlight the distinction between edge state and bulk states, Fig. 2.7(c-e) plots the eigenfunctions of the edge and bulk states. As can be seen, bulk state eigenfunctions occupy the bulk of the lattice, whereas edge state wavefunctions are confined to the perimeter of the lattice. Further, the edge states in the two band-gaps travel with opposite group velocities, circulating clockwise and counterclockwise around the lattice (Fig. 2.7(f-h)). Since the edge states with opposite group velocities have different energies, the edge states are robust against reflection.

More importantly, the edge states are topologically non-trivial. They are always confined to the perimeter of the lattice, irrespective of the shape of the lattice. To characterize their topological order, we use a topological invariant integer called the winding number. The winding number $t_{n}$ for the edgestates in square lattice system can be calculated using the Diophantine equations as $[8,16,29,39]$

$$
n=q s_{n}+p t_{n} ; \quad\left|t_{n}\right| \leq \frac{q}{2}
$$

where $n$ and $s_{n}$ are integers with $n$ being the gap index $(n \leq q-1)$. A nonzero winding number indicates topologically non-trivial states. For example, for $\alpha_{\mathrm{M}}=\frac{1}{4}$, 


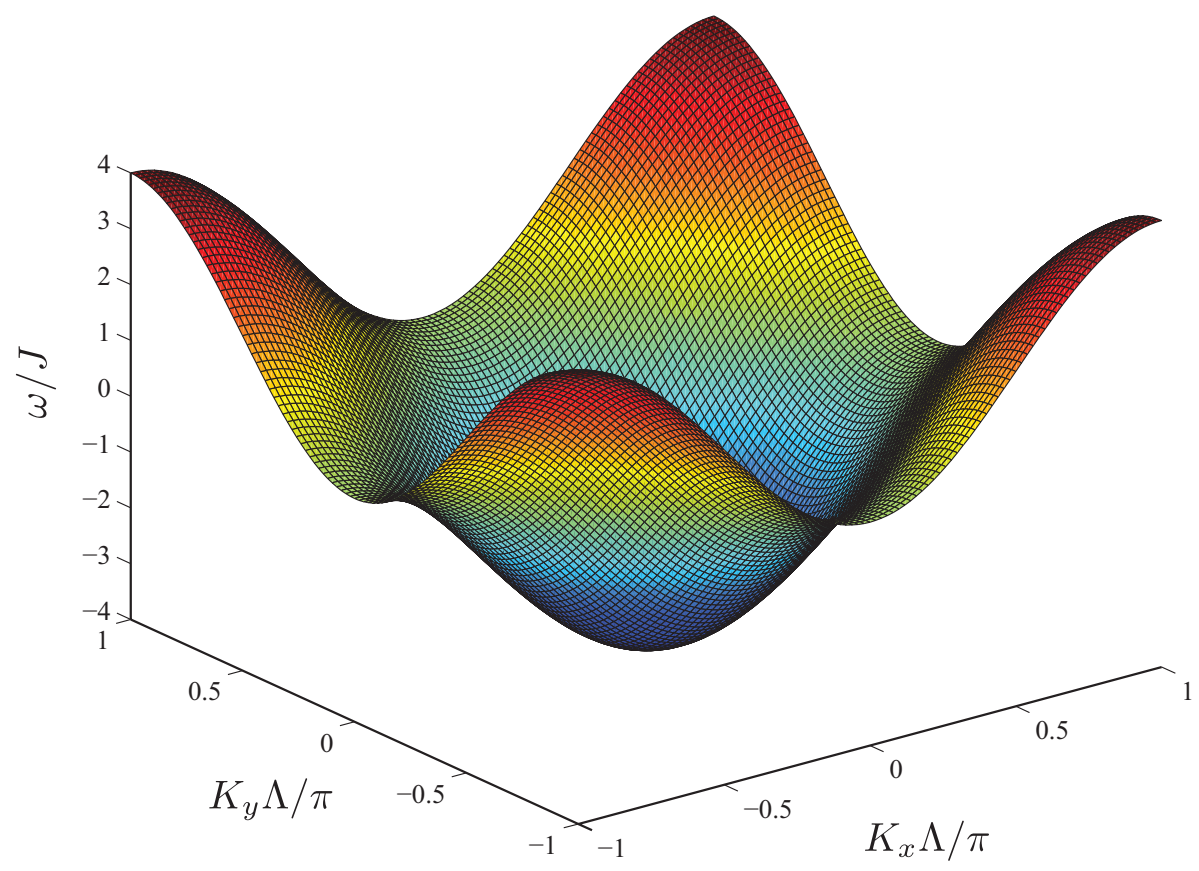

Figure 2.8: Dispersion for an infinite lattice, described by tight-binding Hamiltonian in Eq. 2.52

the first and the third band-gaps have a winding number \pm 1 , respectively, but the second bandgap has a winding number 0 (the winding number for second bandgap is actually not well defined because the bands touch each other [39]). Further, the opposite sign of winding numbers for the two sets of states signifies their clockwise and counter clockwise group velocities. Since the states in the first and third bandgaps are topologically protected, they are robust against fabrication disorders in the lattice. 


\subsubsection{The Dispersion Relation}

Consider an infinite square lattice, with no magnetic field, described by the tight-binding Hamiltonian

$$
H_{0}=\sum_{x, y} \hat{a}_{x, y}^{\dagger} \hat{a}_{x, y}-J\left(\sum_{x, y} \hat{a}_{x+1, y}^{\dagger} \hat{a}_{x, y}+\hat{a}_{x, y}^{\dagger} \hat{a}_{x+1, y}+\hat{a}_{x, y+1}^{\dagger} \hat{a}_{x, y}+\hat{a}_{x, y}^{\dagger} \hat{a}_{x, y+1}\right) .
$$

For an infinite lattice, we can employ Bloch's theorem to relate the ladder operators at different lattice sites as

$$
\hat{a}_{x+n_{x}, y+n_{y}}=e^{-i\left(n_{x} K_{x} \Lambda+n_{y} K_{y} \Lambda\right)} .
$$

Using this relation in Eq. 2.52 gives the dispersion relation for this infinite lattice as

$$
\omega=-2 J\left(\cos \left(K_{x} \Lambda\right)+\cos \left(K_{y} \Lambda\right)\right)
$$

Here, we have assumed that the energy of the system is given by $\hbar \omega$ and scaled the site-potential to zero. Fig. 2.8 plots the dispersion relation. This dispersion relation defines the tight-binding Hamiltonian (without the magnetic field).

\subsection{Summary}

In this Chapter, we discussed the quantization of conductance in $2 \mathrm{D}$ electron gas under the influence of a magnetic field. We saw that the quantization of conductance is a direct manifestation of the quantization of energy levels - the Landau levels. We then discussed a tight-binding lattice model of the quantum Hall effect and looked at its energy spectrum. For an infinite lattice, the energy spectrum 
is grouped into bands interleaved with bandgaps. For a finite lattice, edge states appear in the bandgaps. These edge states live on the edge of the lattice and are immune to back-scattering. Finally, we derived the dispersion relation for this system, which we seek to simulate for photons. 


\section{Chapter 3: Ring Resonator}

A ring resonator forms the basic building block of our system which implements a synthetic gauge field for photons. In the simplest sense, a ring resonator is a waveguide folded onto itself. Because of this geometry, only the frequencies $\omega$ which satisfy the relation

$$
\frac{\omega}{c} n_{\mathrm{eff}} L=2 \pi m,
$$

interfere constructively and can exist in the ring. Here $n_{\mathrm{eff}}$ is the effective refractive index in the ring waveguide, $L$ is the length of the ring and $m$ is an integer called the mode number. Usually this ring waveguide is evanescently coupled to one or two linear waveguides which act as input and output ports to the ring (Fig. 3.1). The configuration where the ring is coupled only to one waveguide is called the All-Pass Filter (APF). In an APF, given the ring loss is zero, all wavelengths pass through the filter with equal transmission. However, the wavelengths which are resonant with the ring suffer a higher delay because of multiple round trips inside the ring. For nonzero ring loss, this configuration acts as a notch filter where the transmission for resonant wavelengths is reduced. The other configuration where a ring is coupled to two linear waveguides is called the Add-Drop Filter (ADF). In this configuration, the input to the ring can be from either of the waveguides and depending on the 
(a)

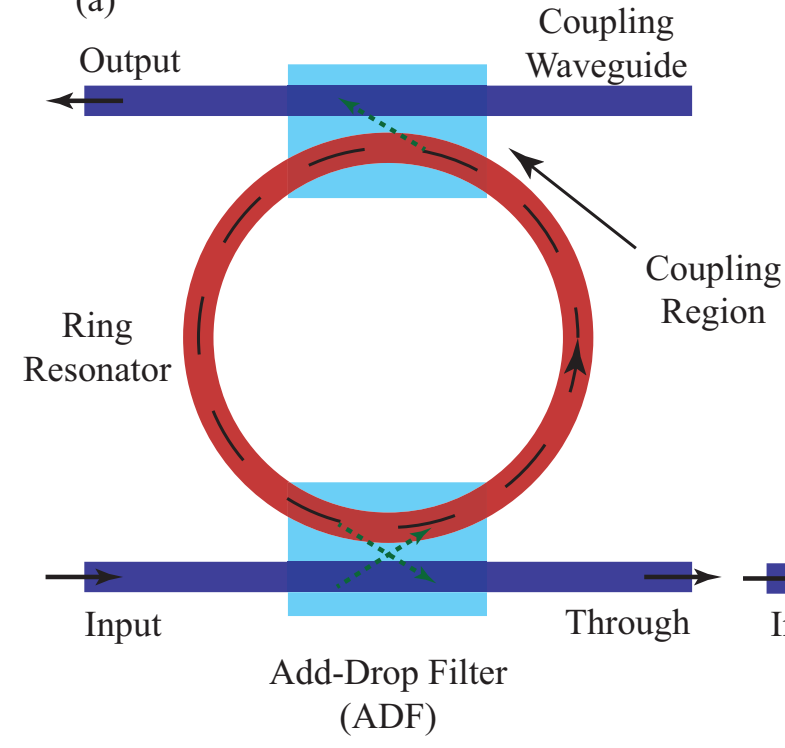

(b)

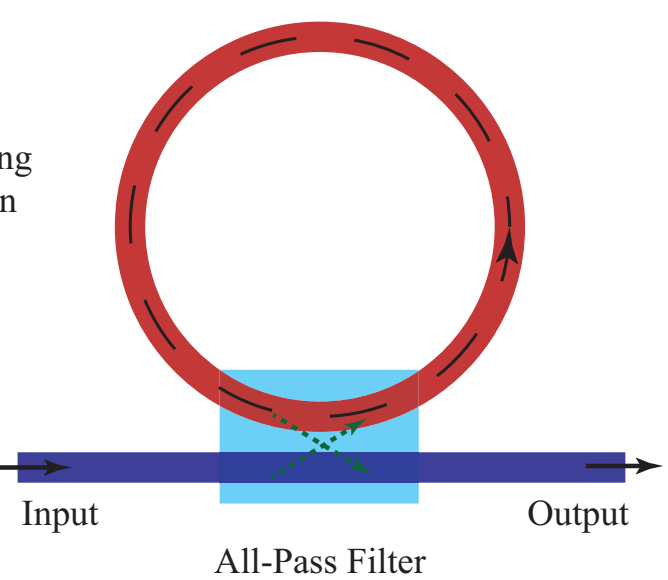

(APF)

Figure 3.1: Schematic of a ring resonator evanescently coupled to input and output waveguides.(a) Add-Drop Filter configuration where the ring is coupled to two waveguides, (b) All-Pass Filter configuration where the ring is coupled to only one waveguide.

input waveguide used and the input wavelength, the output appears at one of the ports. If the input is as shown in Fig. 3.1(a), the frequencies which satisfy Eq. 3.1 are directed to the drop port while the other frequencies to the through port.

The ADF and the APF can be analyzed for their transmission and delay spectra using a vigorous transfer matrix method (TMM) or using a simpler singlemode approximation. The TMM yields a solution of the light field at each point in the ring whereas the single-mode approximation treats the ring resonator as a lumped element with a single mode. Accordingly, the TMM gives the complete transmission spectrum of the ring, i.e. the spectrum at all wavelengths, covering all resonances corresponding to different longitudinal modes. On the other hand, the single mode approximation yields a solution only for one mode, that is, only near one resonance. However, the single mode approximation helps develop an 


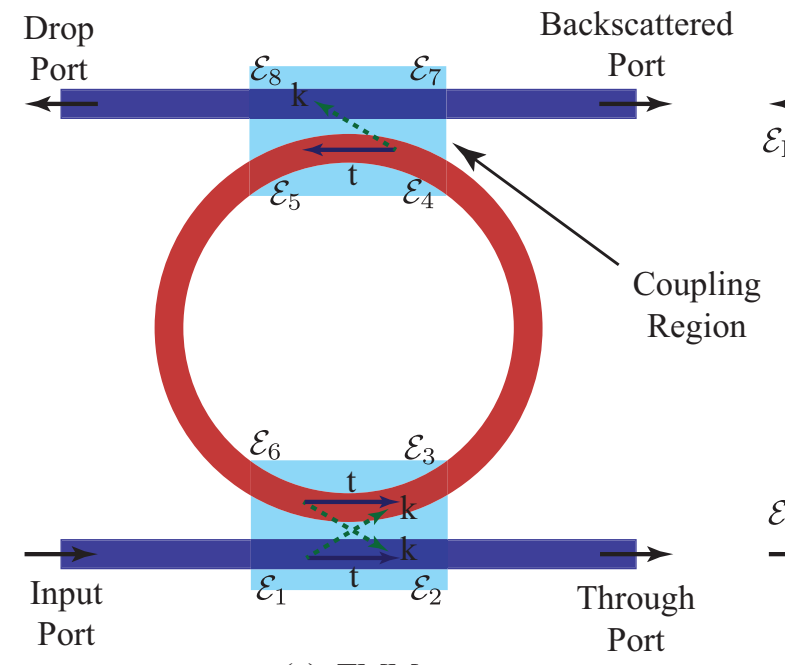

(a) TMM

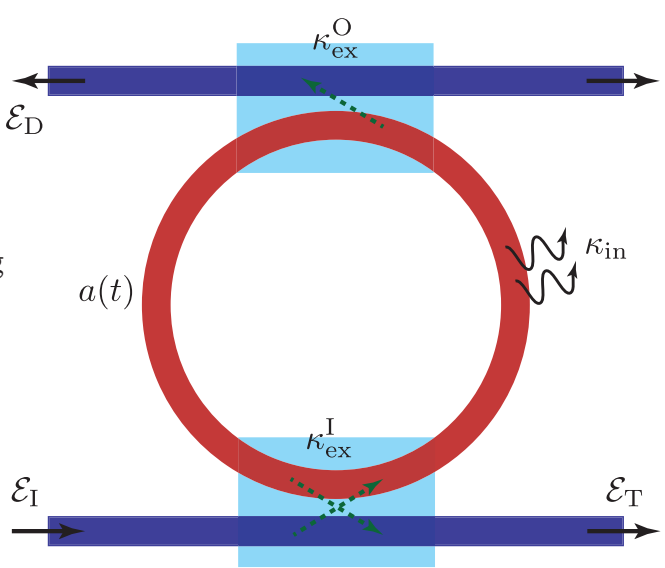

(b) SMA

Figure 3.2: (a) Transfer matrix analysis of a ring resonator. Each coupling region is characterized by a transfer matrix with transmission coefficient $t$ and coupling coefficient $k$. (b) The ring resonator supports a single mode with total energy amplitude $a(t)$ and is coupled to the waveguides with a coupling rate $\kappa_{\text {ex }}^{\mathrm{I}}$ and $\kappa_{\mathrm{ex}}^{\mathrm{O}}$.

intuitive approach to the solution. In the following, we study both these approaches and establish a connection between the two. We will justify the use of the singlemode approximation to study the $2 \mathrm{D}$ system of coupled ring resonators. Also, we will only discuss the ADF configuration since this is the configuration we will later use to characterize our system of coupled resonators. The APF can be analyzed similarly.

\subsection{Transfer Matrix Method (TMM)}

The transfer matrix method uses electric field amplitudes to analyze a ring resonator. We begin with designating the field amplitudes at different points in the ADF as shown in Fig. 3.2(a). The field amplitude here is a function of the position in the ring waveguide, but the transverse field profile in the ring waveguide 
is not considered, assuming the transverse field profile remains constant throughout the ring. Fig. 3.2 also shows the port designations we will use. Light input at the input port and resonant with the ring appears at the drop port after travelling multiple times through the ring. The input light frequencies not resonant with the ring appear at the through port. Light which gets backscattered in the ring at the coupling regions or due to waveguide surface roughness will appear at the backscattered port. The ring can, in general, support two degenerate, clockwise and counterclockwise propagating. We can selectively excite a particular mode by choosing the appropriate input port. However, a significant backscattering in the ring waveguide can result in a coupling between the two modes and exciting the ring with only one of the modes can still result in the second mode being generated (Appendix A). We will usually excite the ring using only one input and assume the backscattering in our ring waveguides is negligible. Hence in our system only one of the two modes propagate in the rings. For the single, counterclockwise propagating mode, the field amplitudes at the coupling regions are then related using the transfer matrix as [41]

$$
\left(\begin{array}{c}
\mathcal{E}_{2} \\
\mathcal{E}_{3}
\end{array}\right)=\left(\begin{array}{cc}
t & i k \\
i k & t
\end{array}\right)\left(\begin{array}{c}
\mathcal{E}_{1} \\
\mathcal{E}_{6}
\end{array}\right)
$$

and

$$
\left(\begin{array}{c}
\mathcal{E}_{8} \\
\mathcal{E}_{5}
\end{array}\right)=\left(\begin{array}{cc}
t & i k \\
i k & t
\end{array}\right)\left(\begin{array}{c}
\mathcal{E}_{7} \\
\mathcal{E}_{4}
\end{array}\right) .
$$

Here $t$ is the field transmission coefficient through the coupling region and $k$ is the coupling coefficient. The $i$ in the transfer matrix represents the phase shift during 
coupling and makes the transfer matrix unitary. Therefore, for a coupling region without coupling loss,

$$
t^{2}+k^{2}=1
$$

The other field amplitudes are related using the propagation phase and loss as

$$
\mathcal{E}_{4}=\mathcal{E}_{3} e^{-i \beta \frac{L}{2}} e^{-\alpha \frac{L}{2}}
$$

and

$$
\mathcal{E}_{6}=\mathcal{E}_{5} e^{-i \beta \frac{L}{2}} e^{-\alpha \frac{L}{2}}
$$

where

$$
\beta=\omega \frac{n_{\mathrm{eff}}(\omega)}{c}
$$

is the propagation constant and $\alpha$ is field decay coefficient per unit length. The frequency dependence of $n_{\text {eff }}$ accounts for the material and waveguide dispersion. These relations describe the free propagation of fields inside the ring resonator.

These are a set of 6 equations with 8 variables. For no input at the backscattering port, i.e. $\mathcal{E}_{7}=0$, the remaining fields can be solved in terms of the incoming field $\mathcal{E}_{1}$. On solving these, the drop and through fields are,

$$
\begin{gathered}
\mathcal{E}_{\mathrm{T}}=\mathcal{E}_{2}=\frac{t-t e^{-i \beta L} e^{-\alpha L}}{1-t^{2} e^{-i \beta L} e^{-\alpha L}} \mathcal{E}_{1} \\
\mathcal{E}_{\mathrm{D}}=\mathcal{E}_{8}=-\frac{k^{2} e^{-i \beta \frac{L}{2}} e^{-\alpha \frac{L}{2}}}{1-t^{2} e^{-i \beta L} e^{-\alpha L}} \mathcal{E}_{1} .
\end{gathered}
$$

The power transmission coefficients for the through and drop port are then,

$$
\begin{aligned}
& T_{\mathrm{T}}=\left|\frac{\mathcal{E}_{\mathrm{T}}}{\mathcal{E}_{1}}\right|^{2}=\frac{t^{2}\left(1+e^{-2 \alpha L}-2 e^{-\alpha L} \cos (\beta L)\right)}{1+t^{4} e^{-2 \alpha L}-2 t^{2} e^{-\alpha L} \cos (\beta L)} \\
& T_{\mathrm{D}}=\left|\frac{\mathcal{E}_{\mathrm{D}}}{\mathcal{E}_{1}}\right|^{2}=\frac{k^{4} e^{-\alpha L}}{1+t^{4} e^{-2 \alpha L}-2 t^{2} e^{-\alpha L} \cos (\beta L)}
\end{aligned}
$$



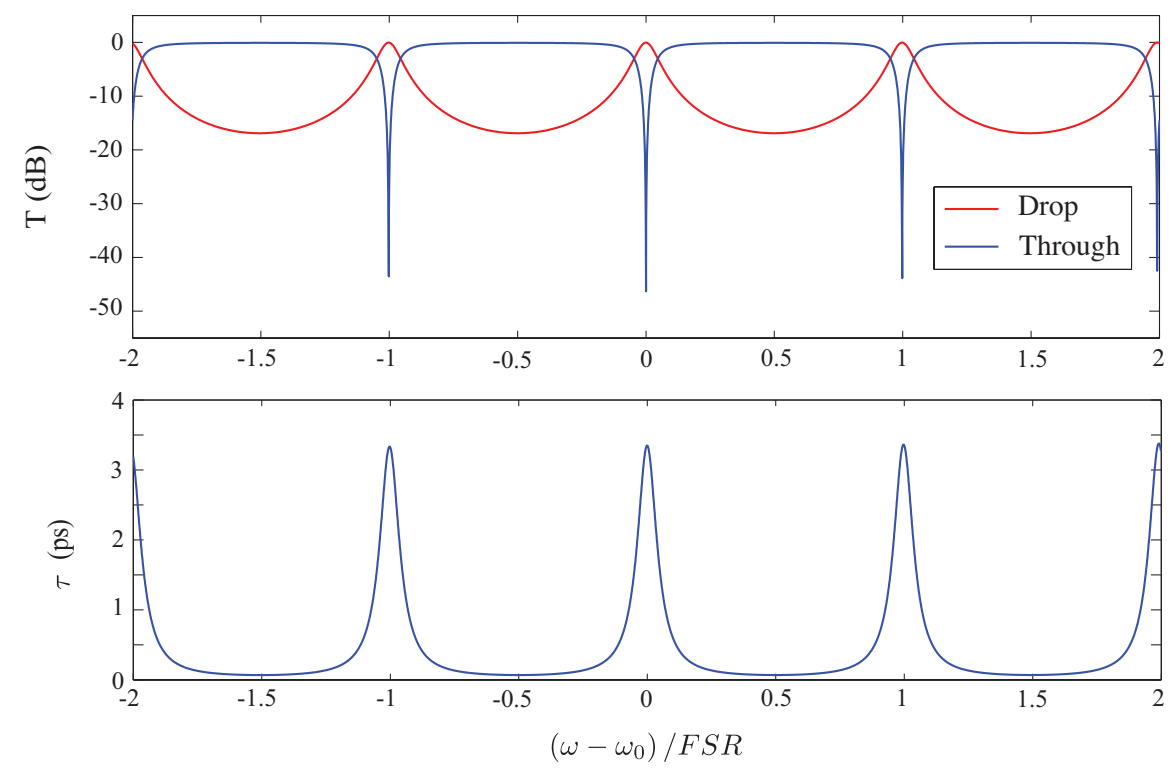

Figure 3.3: (a)Transmission spectrum at the drop and through ports as a function of input frequency, calculated using transfer matrix method. At resonance, the output is maximum at the drop port is minimum at the through port. The spectrum also shows multiple FSRs corresponding to different mode numbers. (b)

Typical power transmission spectra at the drop and through ports are shown in Fig. 3.3(a). The spectra show resonance peaks corresponding to different mode numbers, i.e., at multiples of the free spectral ranges (FSR). FSR is defined as the frequency spacing between the longitudinal modes of the ring resonator and is given as

$$
F S R=2 \pi \frac{v_{\mathrm{g}}}{L}
$$

Here we have included the frequency dependence of $n_{\text {eff }}$, which yields $v_{\mathrm{g}}=\frac{c}{n_{\mathrm{g}}}$, where $n_{\mathrm{g}}=n_{\mathrm{eff}}(\omega)+\omega \frac{d n_{\mathrm{eff}}(\omega)}{d \omega}$.

At resonance, i.e., when $\beta L=2 \pi m$, the minimum transmission at the through port and maximum transmission at the drop port are

$$
T_{\mathrm{T}}^{\mathrm{RES}}=t^{2} \frac{\left(1-e^{-\alpha L}\right)^{2}}{\left(1-t^{2} e^{-\alpha L}\right)^{2}}
$$


and

$$
T_{\mathrm{D}}^{\mathrm{RES}}=\frac{k^{4} e^{-\alpha L}}{\left(1-t^{2} e^{-\alpha L}\right)^{2}}
$$

Therefore, we see that in the absence of loss $(\alpha=0)$, at resonance, all the input light appears at the drop port $\left(T_{\mathrm{D}}^{\mathrm{RES}}=1\right)$. However, in the presence of loss, at resonance, the power transfer to the drop port is not complete and some power remains at the through port. Away from resonance, $T_{\mathrm{T}}$ is maximum and $T_{\mathrm{D}}$ is minimum. We can also verify that in the absence of loss, $T_{\mathrm{T}}+T_{\mathrm{D}}=1$ at all input frequencies.

Further, we can calculate the delay at the drop port using a derivative of the phase $\phi_{\mathrm{D}}$ of the field as

$$
\tau_{\mathrm{D}}=-\frac{d \phi_{\mathrm{D}}}{d \omega}
$$

where $\phi_{\mathrm{D}}=\frac{\mathcal{E}_{\mathrm{D}}}{\left|\mathcal{E}_{\mathrm{D}}\right|}$. The delay thus calculated is called the Wigner delay [42]. The delay spectrum at the drop port is shown in Fig. 3.3(b). As expected, we observe that the delay is maximum at resonance because the light goes through multiple round-trips of the ring resonator.

\subsection{Single Mode Approximation (SMA)}

In the single mode approximation, we treat the ring resonator as a lumped element consisting a single mode, with total energy stored in the ring at time $t$ being $|a(t)|^{2}$, where $a(t)$ is the energy amplitude of the mode (Fig. 3.2(b)). This total energy content of the ring can be related to the field amplitude $\mathcal{E}_{\mathrm{R}}(t)$ travelling 
inside the ring as [43]

$$
|a(t)|^{2}=\left|\mathcal{E}_{\mathrm{R}}(t)\right|^{2} \frac{L}{v_{\mathrm{g}}}
$$

where $v_{\mathrm{g}}$ is the group velocity. We can now analyze the ADF using a differential equation which dictates the time evolution of the energy amplitude $a(t)$ and is given as

$$
\frac{d a}{d t}=i \omega_{0} a-\frac{a}{\tau}-\sqrt{2 \kappa_{\mathrm{ex}}^{\mathrm{I}}} \mathcal{E}_{\mathrm{I}}(t)
$$

Here $\omega_{0}$ is the resonance frequency of the ring resonator and the last term on the right is the coupling of the ring to input field $\mathcal{E}_{\mathrm{I}}(t)$, with a coupling rate $\kappa_{\text {ex }}^{\mathrm{I}}$. The second terms on the right represents the decay of ring energy with a rate constant $\tau$, because of resonator losses and coupling to output waveguides (drop and through ports). The decay rate $\tau$ of the ring can therefore be decomposed as

$$
\frac{1}{\tau}=\left(\frac{1}{\tau_{\mathrm{ex}}^{\mathrm{I}}}+\frac{1}{\tau_{\mathrm{ex}}^{\mathrm{O}}}+\frac{1}{\tau_{\mathrm{in}}}\right)=\left(\kappa_{\mathrm{ex}}^{\mathrm{I}}+\kappa_{\mathrm{ex}}^{\mathrm{O}}+\kappa_{\mathrm{in}}\right)
$$

where $\tau_{\text {ex }}^{\mathrm{I}}, \tau_{\text {ex }}^{\mathrm{O}}$ and $\tau_{\text {in }}$ are the decay rate constants due to coupling to the through port, the drop port and decay due to resonator loss, respectively. $\kappa_{\text {ex }}^{\mathrm{I}}, \kappa_{\text {ex }}^{\mathrm{O}}$ and $\kappa_{\mathrm{in}}$ are the corresponding decay rates - the inverse of decay rate constants. $\kappa_{\text {ex }}^{\mathrm{I}}$ and $\kappa_{\mathrm{ex}}^{\mathrm{O}}$ are dictated by the coupling strength of the ring to input and output waveguides, whereas, $\kappa_{\text {in }}$ represents the energy loss due to waveguide surface roughness scattering and waveguide bending losses.

For a plane wave input excitation of the form $\mathcal{E}_{\mathrm{I}}(t)=\mathcal{E}_{\mathrm{I}} e^{-i \omega t}$, the steady state solution for the ring energy amplitude can be derived by setting $\frac{d a}{d t}=0$ as,

$$
a=\frac{-\sqrt{2 \kappa_{\mathrm{ex}}^{\mathrm{I}}}}{i\left(\omega-\omega_{0}\right)+\left(\kappa_{\mathrm{ex}}^{\mathrm{I}}+\kappa_{\mathrm{ex}}^{\mathrm{O}}+\kappa_{\mathrm{in}}\right)} \mathcal{E}_{\mathrm{T}}
$$


The output field amplitude at drop port is then [44]

$$
\mathcal{E}_{\mathrm{D}}=\sqrt{2 \kappa_{\mathrm{ex}}^{\mathrm{O}}} a=\frac{-2 \sqrt{\kappa_{\mathrm{ex}}^{\mathrm{I}} \kappa_{\mathrm{ex}}^{\mathrm{O}}}}{i\left(\omega-\omega_{0}\right)+\left(\kappa_{\mathrm{ex}}^{\mathrm{I}}+\kappa_{\mathrm{ex}}^{\mathrm{O}}+\kappa_{\mathrm{in}}\right)} \mathcal{E}_{\mathrm{I}}
$$

and at the through port is

$$
\mathcal{E}_{\mathrm{T}}=\mathcal{E}_{\mathrm{I}}-\sqrt{2 \kappa_{\mathrm{ex}}^{\mathrm{I}}} a=\frac{i\left(\omega-\omega_{0}\right)+\left(\kappa_{\mathrm{ex}}^{\mathrm{O}}-\kappa_{\mathrm{ex}}^{\mathrm{I}}+\kappa_{\mathrm{in}}\right)}{i\left(\omega-\omega_{0}\right)+\left(\kappa_{\mathrm{ex}}^{\mathrm{I}}+\kappa_{\mathrm{ex}}^{\mathrm{O}}+\kappa_{\mathrm{in}}\right)} \mathcal{E}_{\mathrm{I}}
$$

Using these equations, we derive the power transmission at the drop and through port as

$$
T_{\mathrm{D}}=\left|\frac{\mathcal{E}_{D}}{\mathcal{E}_{\mathrm{I}}}\right|^{2}=\frac{4 \kappa_{\mathrm{ex}}^{\mathrm{I}} \kappa_{\mathrm{ex}}^{\mathrm{O}}}{\left(\omega-\omega_{0}\right)^{2}+\left(\kappa_{\mathrm{ex}}^{\mathrm{I}}+\kappa_{\mathrm{ex}}^{\mathrm{O}}+\kappa_{\mathrm{in}}\right)^{2}}
$$

and

$$
T_{\mathrm{T}}=\left|\frac{\mathcal{E}_{T}}{\mathcal{E}_{\mathrm{I}}}\right|^{2}=\frac{\left(\omega-\omega_{0}\right)^{2}+\left(\kappa_{\mathrm{ex}}^{\mathrm{O}}-\kappa_{\mathrm{ex}}^{\mathrm{I}}+\kappa_{\mathrm{in}}\right)^{2}}{\left(\omega-\omega_{0}\right)^{2}+\left(\kappa_{\mathrm{ex}}^{\mathrm{I}}+\kappa_{\mathrm{ex}}^{\mathrm{O}}+\kappa_{\mathrm{in}}\right)^{2}} .
$$

A typical transmission spectrum at the drop and through ports is shown in Fig. 3.4(a). Since we assumed only a single mode travelling inside the ring, unlike TMM, the transmission spectrum exhibits only one resonance. At resonance, i.e. when $\omega=\omega_{0}$, the transmission is maximum at drop port and minimum at the through port given by

$$
\begin{aligned}
T_{\mathrm{D}}^{\mathrm{RES}} & =\frac{4 \kappa_{\mathrm{ex}}^{\mathrm{I}} \kappa_{\mathrm{ex}}^{\mathrm{O}}}{\left(\kappa_{\mathrm{ex}}^{\mathrm{I}}+\kappa_{\mathrm{ex}}^{\mathrm{O}}+\kappa_{\mathrm{in}}\right)^{2}} \\
T_{\mathrm{T}}^{\mathrm{RES}} & =\frac{\left(\kappa_{\mathrm{ex}}^{\mathrm{O}}-\kappa_{\mathrm{ex}}^{\mathrm{I}}+\kappa_{\mathrm{in}}\right)^{2}}{\left(\kappa_{\mathrm{ex}}^{\mathrm{I}}+\kappa_{\mathrm{ex}}^{\mathrm{O}}+\kappa_{\mathrm{in}}\right)^{2}} .
\end{aligned}
$$

As we saw using TMM, for lossless ring $\left(\kappa_{\mathrm{in}}=0\right)$ and $\kappa_{\mathrm{ex}}^{\mathrm{I}}=\kappa_{\mathrm{ex}}^{\mathrm{O}}$, the transmission at the drop port is equal to unity at resonance and zero at the through port. Away from resonance, the transmission at the through port is unity and negligible at the drop port. Also at all frequencies, in absence of loss, $T_{\mathrm{T}}+T_{\mathrm{D}}=1$. 

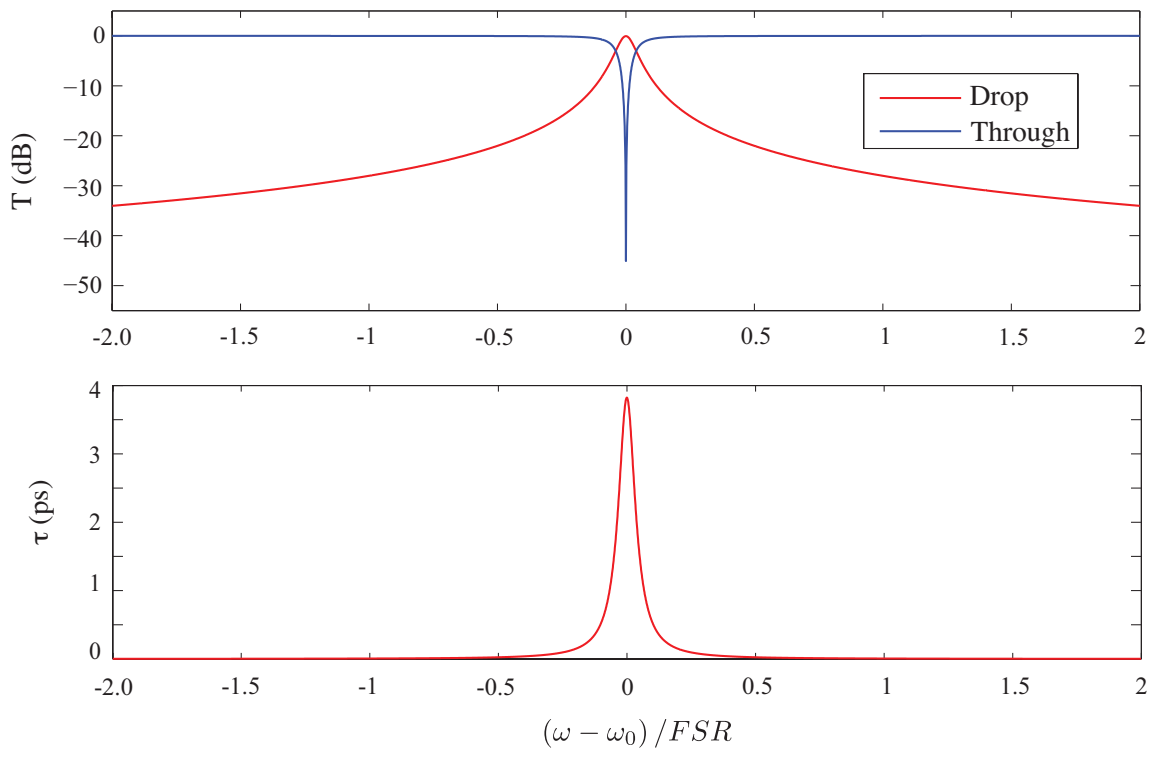

Figure 3.4: Transmission spectrum at the drop and through ports as a function of input frequency, simulated using single-mode approximation. As with transfer matrix analysis, at resonance, the transmission is maximum at the drop port. However, this approximation yields only one resonance peak of the resonator. (b) Delay (Wigner) spectrum at the drop port.

The Wigner delay at the drop port can be calculated using Eq. 3.15 to be

$$
\tau_{\mathrm{D}}=-\frac{d \phi_{\mathrm{D}}}{d \omega}=\frac{2 \sqrt{\kappa_{\mathrm{ex}}^{\mathrm{I}} \kappa_{\mathrm{ex}}^{\mathrm{O}}}}{\left(i\left(\omega-\omega_{0}\right)+\left(\kappa_{\mathrm{ex}}^{\mathrm{I}}+\kappa_{\mathrm{ex}}^{\mathrm{O}}+\kappa_{\mathrm{in}}\right)\right)^{2}} .
$$

The maximum delay at resonance is

$$
\tau_{\mathrm{D}}^{\mathrm{RES}}=\frac{2 \sqrt{\kappa_{\mathrm{ex}}^{\mathrm{I}} \kappa_{\mathrm{ex}}^{\mathrm{O}}}}{\left(\kappa_{\mathrm{ex}}^{\mathrm{I}}+\kappa_{\mathrm{ex}}^{\mathrm{O}}+\kappa_{\mathrm{in}}\right)^{2}} .
$$

For $\left(\kappa_{\mathrm{in}}=0\right)$ and $\kappa_{\mathrm{ex}}^{\mathrm{I}}=\kappa_{\mathrm{ex}}^{\mathrm{O}}=\kappa_{\mathrm{ex}}$, this relation simplifies to

$$
\tau_{\mathrm{D}}^{\mathrm{RES}}=\frac{1}{2 \kappa_{\mathrm{ex}}}
$$

\subsection{Equivalence between TMM and SMA}

We see that in the transfer matrix method, the ADF is characterized by the coupling coefficients $t, k$ and the loss coefficient $\alpha$. In the single-mode approximation, 
the same $\mathrm{ADF}$ is characterized by coupling rate $\kappa_{\mathrm{ex}}$ (assuming $\kappa_{\mathrm{ex}}^{\mathrm{I}}=\kappa_{\mathrm{ex}}^{\mathrm{O}}=\kappa_{\mathrm{ex}}$ ) and the loss rate $\kappa_{\text {in }}$. We can therefore derive an equivalence between these two sets of parameters. To do so, we first consider the FWHM $\left(\Delta \omega_{0}\right)$ of the transmission peak at the drop port. For the SMA, the FWHM (in angular frequency units) can be calculated from Eq. 3.22 to be

$$
\Delta \omega_{0}^{\mathrm{SMA}}=2\left(2 \kappa_{\mathrm{ex}}+\kappa_{\mathrm{in}}\right)
$$

Similarly, in the TMM, the FWHM (in angular frequency units) of the drop port spectrum from Eq. 3.11 is

$$
\Delta \omega_{0}^{\mathrm{TMM}}=\frac{2 v_{\mathrm{g}}}{L}\left(e^{\alpha L}-t^{2} e^{-\alpha L}\right)
$$

For small $\alpha, k$, so that the bandwidth of the drop port is small compared to the FSR of the resonator, this can be simplified to

$$
\Delta \omega_{0}^{\mathrm{TMM}}=\frac{2 v_{\mathrm{g}}}{L}\left(k^{2}+\alpha L\right)
$$

Thus, equating the two relations, for a ring with low loss and small coupling to the waveguides,

$$
\kappa_{\mathrm{ex}}=\frac{k^{2}}{2} \frac{v_{\mathrm{g}}}{L}
$$

and

$$
\kappa_{\text {in }}=\alpha v_{\mathrm{g}} .
$$

These relations thus establish the equivalence between the two approaches to analyze the ring resonator in the weak coupling regime. A comparison of the transmission and delay spectra calculated using the two approaches is shown in 

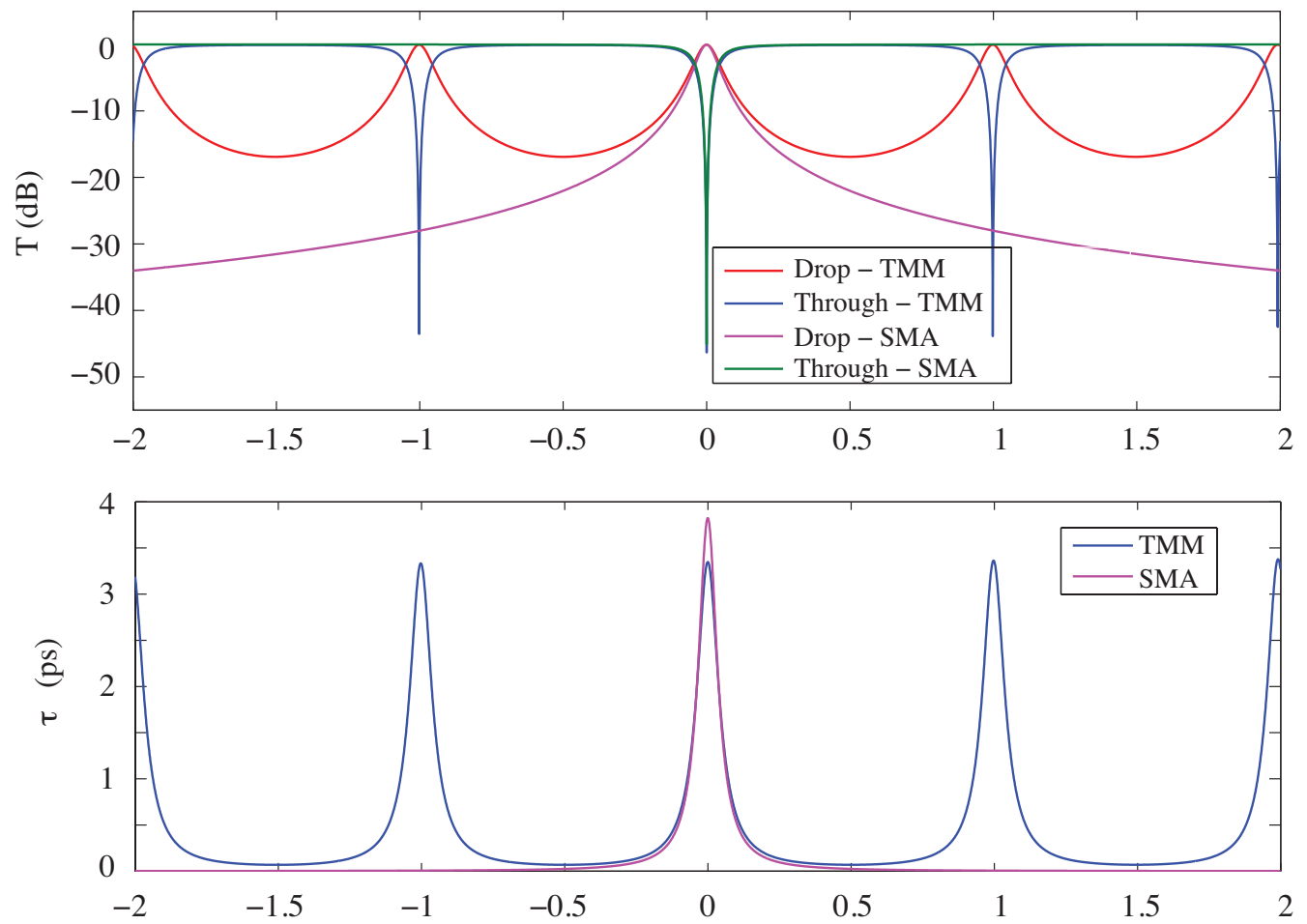

Figure 3.5: Comparison of (a) the transmission spectrum, (b) the delay spectrum, of a ring resonator simulated using TMM and SMA. The SMA and TMM match very well for $\omega-\omega_{0} \ll F S R$, i.e. when the resonance bandwidth is much less than the FSR of the ring. As the difference $\omega-\omega_{0}$ becomes comparable to FSR, the discrepancy increases. 
Fig. 3.5. We find that the single mode approximation is strictly valid when the ring resonator is coupled weakly to the input/output waveguides and the resonator losses are small, i.e., $\kappa_{\mathrm{ex}}, \kappa_{\mathrm{in}} \ll F S R$. In other words, this means that the single mode approximation holds when the bandwidth of the transmission spectrum near a resonance $\left(4 \kappa_{\text {ex }}+2 \kappa_{\text {in }}\right)$ is much less than the FSR.

We can also derive these expressions considering the ring energy decay rates. In the SMA, neglecting loss and in the absence of external input coupling, the energy decays as

$$
|a(t)|^{2}=|a(0)|^{2} e^{-2 t / \tau}
$$

where $\tau$ is defined as in Eq. 3.18. In the absence of loss, the energy decays because the ring is coupled to through and drop ports. Now since the through and drop port waveguide couplers are characterized by coupling constant $k$, the total power output at through and drop ports is

$$
\left|\mathcal{E}_{\mathrm{T}}\right|^{2}+\left|\mathcal{E}_{\mathrm{D}}\right|^{2}=k^{2}\left|\mathcal{E}_{\mathrm{R}}(t)\right|^{2}+k^{2}\left|\mathcal{E}_{\mathrm{R}}(t)\right|^{2}=2 k^{2}|a(t)|^{2} \frac{v_{\mathrm{g}}}{L}
$$

In the SMA, with the coupling constant $\kappa_{e x}$, these fields are

$$
\left|\mathcal{E}_{\mathrm{T}}\right|^{2}+\left|\mathcal{E}_{\mathrm{D}}\right|^{2}=2 \kappa_{\mathrm{ex}}|a(t)|^{2}+2 \kappa_{\mathrm{ex}}|a(t)|^{2}
$$

Comparing both expressions we get

$$
\kappa_{\mathrm{ex}}=\frac{k^{2}}{2} \frac{v_{\mathrm{g}}}{L}
$$

which is same as derived earlier (Eq. 3.32).

Similarly, we can equate the ring energy loss in a time duration $t$, when the ring is not coupled to any of the waveguides and the ring loses energy only because 
of loss waveguide surface roughness scattering and absorption loss. Doing so yields the equation,

$$
|a(t)|^{2} e^{-2 t \kappa_{\mathrm{in}}}=|a(t)|^{2} e^{-2 \alpha v_{\mathrm{g}} t}
$$

which gives,

$$
\kappa_{\text {in }}=\alpha v_{\mathrm{g}}
$$

\subsection{Finesse and Quality Factor of the Resonator}

The Finesse $(F)$ of a resonator is a measure of the number of round trips the light makes before leaking out of the ring. It is defined as the ratio of the free spectral range to the resonance bandwidth and can also be written as the ratio of photon lifetime in the ring to the round-trip time.

$$
F=\frac{F S R}{\Delta \omega_{0}}=2 \pi \frac{\tau_{\mathrm{P}}}{\tau_{\mathrm{R}}}
$$

Using (3.18) and (3.22), the photon lifetime $\tau_{\mathrm{P}}$ (rate constant for energy decay) in the ring is

$$
\tau_{\mathrm{P}}=\tau / 2=\frac{1}{2\left(2 \kappa_{\mathrm{ex}}+\kappa_{\mathrm{in}}\right)}=\frac{1}{\Delta \omega_{0}^{\mathrm{SMA}}}=2 \tau_{\mathrm{D}}^{\mathrm{RES}},
$$

and the ring round trip time $\tau_{\mathrm{R}}$ is simply

$$
\tau_{\mathrm{R}}=\frac{L}{v_{\mathrm{g}}}
$$

Using above expressions, the Finesse of the ADF is calculated to be

$$
F=\frac{\pi v_{\mathrm{g}}}{L\left(2 \kappa_{\mathrm{ex}}+\kappa_{\mathrm{in}}\right)} .
$$


The quality factor $Q$ is a measure of the ring resonator losses and is defined as

$$
Q=\omega_{0} \frac{\text { Energy stored in the ring }}{\text { Energy loss rate }} .
$$

The energy stored in the ring is simply $|a|^{2}$. The energy loss rate of the ring is in fact the power loss, which includes power lost to input/output waveguides and the intrinsic resonator loss such as the waveguide scattering. Using (3.36) and (3.38), the rate of energy loss (or the power loss) from the ring is

$$
P_{\mathrm{L}}=2\left(2 \kappa_{\mathrm{ex}}+\kappa_{\text {in }}\right)\left|a^{2}\right|
$$

The quality factor for the ADF is then

$$
Q=\omega_{0} \frac{\left|a^{2}\right|}{2\left(2 \kappa_{\mathrm{ex}}+\kappa_{\mathrm{in}}\right)\left|a^{2}\right|}
$$

Using (3.29), the quality factor can also be written as

$$
Q=\frac{\omega_{0}}{\Delta \omega}=m F
$$

where $m$ is the mode number corresponding to $\omega_{0}$. This is the loaded quality factor for the ring since it includes the ring energy loss because of coupling to input and output waveguides.

\subsection{Summary}

In this chapter, we discussed the two approaches to analyze a ring resonator the rigorous transfer matrix method and the more intuitive, single mode approximation. We derived an equivalence between the parameters defining a ring resonator 
in the two approaches. Most importantly, we justified that a ring resonator can be treated as a lumped element with a single mode when the ring is excited by frequencies near its resonance. We use this approximation to simulate the $2 \mathrm{D}$ lattice of tight-binding model for photons. 


\section{Chapter 4: Synthetic Magnetic Field for Photons}

The presence of a magnetic field in a two dimensional electron gas leads to the emergence of topologically robust, reflection free, chiral edge states. Implementing analogs of topological edge states in photonic systems is very desirable from the viewpoint of achieving, on-chip, one-way waveguides. But, at optical frequencies, magneto-optic effects - the only source of coupling between photons and magnetic fields is very weak. In this chapter, we describe how we can engineer a synthetic magnetic field for photons using a 2D lattice of ring resonators and hence achieve topologically ordered edge states. We also discuss an application of these edge states as robust optical delay lines in photonic integrated circuits

\subsection{Coupled Ring Resonators}

In the single-mode approximation, a ring resonator can be treated as a lumped element described by an energy amplitude. Using this fact and taking a clue from the lattice model of the quantum Hall effect, we consider a two-dimensional lattice of ring resonators (Fig. 4.1). The ring resonators form a square lattice and act as lattice sites to which photons are bound - analogous to electrons bound to a periodic potential on lattice sites. These site ring resonators are coupled by evanescent fields 
in the ring waveguides, to their nearest neighbors through link rings. These link resonators have slightly longer length than the main ring resonators. This extra length results in a phase shift of $\pi$ and thus makes the link resonators anti-resonant to the main site ring resonators. Thus for light in the resonance pass band of the site rings, the link resonators do not store any energy and hence act simply as connecting waveguides and not as resonators. More importantly, this coupling of resonators allows the photons to hop from one site ring to a neighboring site, simulating a tight-binding model where electrons hop between nearest neighbors. This 2D lattice of coupled resonators can then described by the same tight-binding Hamiltonian which describes a 2D electron gas, but without a magnetic field.

To introduce a synthetic magnetic field into this system, we use the fact that the effect of an external magnetic field is equivalent to the presence of noncommuting lattice translation operators. In other words, the accumulated phase around a loop, the sign of which depends on the direction of travel around the loop, gives rise to what can be thought of as a synthetic magnetic field for photons. In the following, we discuss how this magnetic field can be realized by appropriate placement of the link rings. We derive the dispersion relation for this $2 \mathrm{D}$ system of coupled ring resonators and show that it is exactly the same as that of the tightbinding Hamiltonian. 


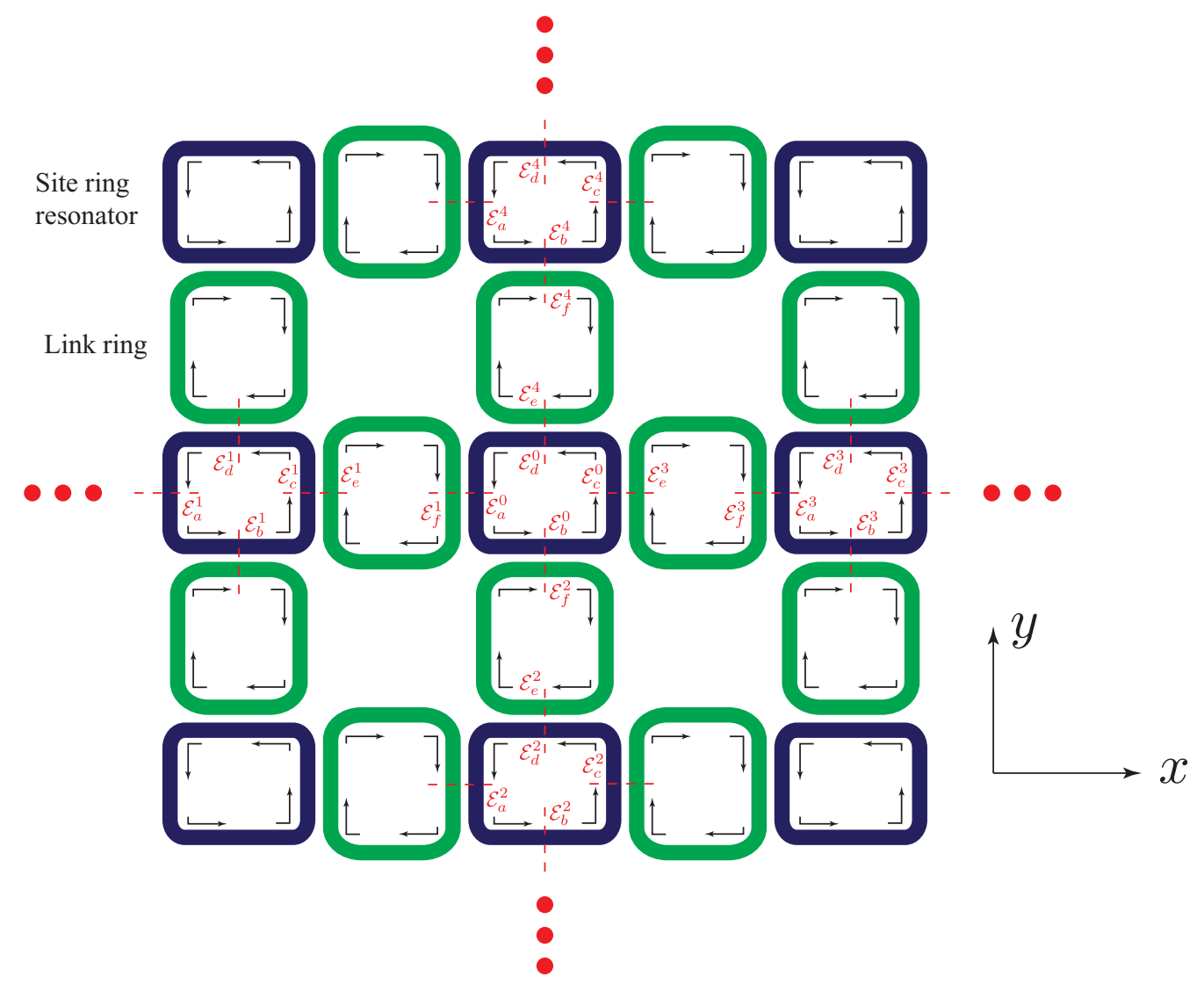

Figure 4.1: A 2D square lattice of ring resonators to simulate the tight-binding model without a magnetic field. The site ring resonators (blue) act as lattice sites and the photons hop from one site to another using link rings (green). Field amplitudes are labeled and discussed in the text. Subscripts indicate specific rings described in Eqs. 4.1-4.11. 


\subsubsection{Tight-Binding Model without Magnetic Field}

To show that the 2D system of coupled site and link resonators in Fig. 4.1 indeed simulates the tight-binding Hamiltonian, without a magnetic field, we consider an infinite lattice of such resonators. An infinite lattice allows us to use Bloch's theorem to relate field amplitudes in different rings. We use the transfer matrix approach and label the field amplitudes as shown in Fig. 4.1. We treat each coupling region between neighboring rings (site and link rings) as a point-like interaction and the field amplitudes are defined at the outputs of the waveguide coupling region (i.e. where two rings run tangent to each other). As an example, the coupling region to the left of the center-most ring in Fig. 4.1 forms a directional coupler and is described by a transfer matrix which relates the outgoing field amplitudes to the incoming field amplitudes as

$$
\left(\begin{array}{c}
\mathcal{E}_{a}^{0} \\
\mathcal{E}_{f}^{1}
\end{array}\right)=\left(\begin{array}{cc}
t & i \kappa \\
i \kappa & t
\end{array}\right)\left(\begin{array}{c}
\mathcal{E}_{d}^{0} e^{-i \beta \frac{L_{\mathrm{SR}}}{4}} \\
\mathcal{E}_{e}^{1} e^{-i \beta \frac{L_{\mathrm{LR}}}{2}}
\end{array}\right)
$$

Here $t$ is the transmission coefficient through a coupling region and $\kappa$ is the coupling coefficient. These parameters can be estimated using finite-difference time domain (FDTD) simulations. The transfer matrix is unitary, meaning that in the absence of coupling loss,

$$
t^{2}+\kappa^{2}=1
$$

The terms $e^{-i \beta \frac{L_{\mathrm{SR}}}{4}}$ and $e^{-i \beta \frac{L_{\mathrm{LR}}}{2}}$ are the phase factors accumulated in travelling $1 / 4^{\text {th }}$ of the total length $L_{\mathrm{SR}}$ of site resonator and $1 / 2$ of the link resonator length $L_{\mathrm{LR}}$, 
respectively. The above equation can also be written as

$$
\left(\begin{array}{c}
\mathcal{E}_{d}^{0} e^{-i \beta \frac{L_{\mathrm{SR}}}{4}} \\
\mathcal{E}_{a}^{0}
\end{array}\right)=\frac{1}{i \kappa}\left(\begin{array}{cc}
-t & 1 \\
-1 & t
\end{array}\right)\left(\begin{array}{c}
\mathcal{E}_{e}^{1} e^{-i \beta \frac{L_{\mathrm{LR}}^{2}}{2}} \\
\mathcal{E}_{f}^{1}
\end{array}\right)
$$

where now the field amplitudes of one ring are described in terms of the field amplitudes of the neighboring ring. Similarly, we can write the fields of the link resonator in terms of the next site resonator (to the left of the resonator described in Eq. 4.3)

$$
\left(\begin{array}{c}
\mathcal{E}_{e}^{1} e^{-i \beta \frac{L_{\mathrm{LR}}}{2}} \\
\mathcal{E}_{f}^{1}
\end{array}\right)=\frac{1}{i \kappa}\left(\begin{array}{cc}
-t & 1 \\
-1 & t
\end{array}\right)\left(\begin{array}{c}
\mathcal{E}_{c}^{1} \\
\mathcal{E}_{b}^{1} e^{-i \beta \frac{L_{\mathrm{SR}}}{4}}
\end{array}\right)
$$

Using these two equations we can relate the fields of one site resonator to the neighbouring site

$$
\left(\begin{array}{c}
\mathcal{E}_{d}^{0} e^{-i \beta \frac{L_{\mathrm{SR}}}{4}} \\
\mathcal{E}_{a}^{0}
\end{array}\right)=\frac{-1}{\kappa^{2}}\left(\begin{array}{cc}
-t & 1 \\
-1 & t
\end{array}\right)\left(\begin{array}{cc}
t e^{-i \beta \frac{L_{\mathrm{LR}}}{2}} & -e^{-i \beta \frac{L_{\mathrm{LR}}}{2}} \\
e^{i \beta \frac{L_{\mathrm{LR}}}{2}} & -t e^{i \beta \frac{L_{\mathrm{LR}}}{2}}
\end{array}\right)\left(\begin{array}{c}
\mathcal{E}_{c}^{1} \\
\mathcal{E}_{b}^{1} e^{-i \beta \frac{L_{\mathrm{SR}}}{4}}
\end{array}\right)
$$

Following the same procedure, we can derive another set of equations relating the field amplitudes of neighboring sites in the vertical direction

$$
\left(\begin{array}{c}
\mathcal{E}_{a}^{0} e^{-i \beta \frac{L_{\mathrm{SR}}}{4}} \\
\mathcal{E}_{b}^{0}
\end{array}\right)=\frac{-1}{\kappa^{2}}\left(\begin{array}{cc}
-t & 1 \\
-1 & t
\end{array}\right)\left(\begin{array}{cc}
t e^{-i \beta \frac{L_{\mathrm{LR}}}{2}} & -e^{-i \beta \frac{L_{\mathrm{LR}}}{2}} \\
e^{i \beta \frac{L_{\mathrm{LR}}}{2}} & -t e^{i \beta \frac{L_{\mathrm{LR}}}{2}}
\end{array}\right)\left(\begin{array}{c}
\mathcal{E}_{d}^{2} \\
\mathcal{E}_{c}^{2} e^{-i \beta \frac{L_{\mathrm{SR}}}{4}}
\end{array}\right)
$$

However, for this infinite lattice, the field amplitudes of the neighboring rings 
are also related using Bloch's theorem [45]

$$
\begin{aligned}
& \mathcal{E}_{b}^{1}=\mathcal{E}_{b}^{0} e^{i K_{x} \Lambda} \\
& \mathcal{E}_{c}^{1}=\mathcal{E}_{c}^{0} e^{i K_{x} \Lambda} \\
& \mathcal{E}_{c}^{2}=\mathcal{E}_{c}^{0} e^{i K_{y} \Lambda} \\
& \mathcal{E}_{d}^{2}=\mathcal{E}_{d}^{0} e^{i K_{y} \Lambda} .
\end{aligned}
$$

Here $K_{x}$ and $K_{y}$ are the lattice momentum components and $\Lambda$ is the lattice constant. Using these relations in Eqs. 4.5-4.6 gives a set of homogenous equations featuring only the field amplitudes of a single site ring

$$
\left(\begin{array}{cccc}
0 & \frac{t}{\kappa^{2}} \Phi_{x} \Phi_{\mathrm{SR}}\left(\Phi_{\mathrm{LR}}-\Phi_{\mathrm{LR}}^{-1}\right) & \frac{1}{\kappa^{2}} \Phi_{x}\left(-t^{2} \Phi_{\mathrm{LR}}+\Phi_{\mathrm{LR}}^{-1}\right) & \Phi_{\mathrm{SR}} \\
1 & \frac{1}{\kappa^{2}} \Phi_{x} \Phi_{\mathrm{SR}}\left(\Phi_{\mathrm{LR}}-t^{2} \Phi_{\mathrm{LR}}\right) & \frac{t}{\kappa^{2}} \Phi_{x}\left(\Phi_{\mathrm{LR}}+\Phi_{\mathrm{LR}}^{-1}\right) & 0 \\
\Phi_{\mathrm{SR}} & 0 & \frac{t}{\kappa^{2}} \Phi_{y} \Phi_{\mathrm{SR}}\left(\Phi_{\mathrm{LR}}-\Phi_{\mathrm{LR}}^{-1}\right) & \frac{1}{\kappa^{2}} \Phi_{y}\left(-t^{2} \Phi_{\mathrm{LR}}+\Phi_{\mathrm{LR}}^{-1}\right) \\
0 & 1 & \frac{1}{\kappa^{2}} \Phi_{y} \Phi_{\mathrm{SR}}\left(\Phi_{\mathrm{LR}}-t^{2} \Phi_{\mathrm{LR}}^{-1}\right) & \frac{t}{\kappa^{2}} \Phi_{y}\left(-\Phi_{\mathrm{LR}}+\Phi_{\mathrm{LR}}^{-1}\right)
\end{array}\right)\left(\begin{array}{c}
a_{0} \\
b_{0} \\
c_{0} \\
d_{0}
\end{array}\right)=0,
$$

where we have used $\Phi_{x}=e^{i K_{x} \Lambda}, \Phi_{y}=e^{i K_{y} \Lambda}, \Phi_{\mathrm{LR}}=e^{-i \beta \frac{L_{\mathrm{SR}}}{4}}$ and $\Phi_{L C R}=e^{-i \beta \frac{L_{\mathrm{LR}}}{2}}$ for brevity. A non-zero solution for this equation implies that the determinant of the square matrix on the left side is zero. Equating this determinant to zero and using the fact that the link resonators are anti-resonant to the site rings, i.e., $\beta L_{\mathrm{LR}}=\beta L_{\mathrm{SR}}+\pi$, results in the following dispersion relation for this $2 \mathrm{D}$ system of coupled ring resonators

$$
\sin \left(\beta L_{\mathrm{SR}}\right)=t \kappa^{2}\left(\cos K_{x} \Lambda+\cos K_{y} \Lambda\right) .
$$

So far we have not used any approximations. When the input field frequency 
$\omega$ is close to the resonance pass-band of the rings

$$
\sin \left(\beta L_{\mathrm{SR}}\right) \approx \beta L_{\mathrm{SR}}=\left(\omega_{0}-\omega\right) \frac{L_{\mathrm{SR}}}{v_{\mathrm{g}}}
$$

Then, in the weak coupling regime $(\kappa<<1$ and $t \approx 1)$, the dispersion relation for this system of coupled ring resonators is given by

$$
\omega=\omega_{0}-2 J\left(\cos K_{x} \Lambda+\cos K_{y} \Lambda\right)
$$

where the coupling rate $J$ is

$$
J=\frac{\kappa^{2}}{2} \frac{v_{\mathrm{g}}}{L_{\mathrm{SR}}}
$$

More importantly, we see that this dispersion relation is exactly the same as that we derived for the tight binding Hamiltonian without the magnetic field (Eq. 2.54)

$$
H_{0}=\sum_{x, y} \hat{a}_{x, y}^{\dagger} \hat{a}_{x, y}-J\left(\sum_{x, y} \hat{a}_{x+1, y}^{\dagger} \hat{a}_{x, y}+\hat{a}_{x, y}^{\dagger} \hat{a}_{x+1, y}+\hat{a}_{x, y+1}^{\dagger} \hat{a}_{x, y}+\hat{a}_{x, y}^{\dagger} \hat{a}_{x, y+1}\right)
$$

Therefore, this system of coupled resonators indeed simulates the tight-binding Hamiltonian for electrons in the weak coupling regime and for input field frequencies close to the resonance, i.e., when the single-mode approximation for a ring resonator is valid. We therefore see that, using the above Hamiltonian, this $2 \mathrm{D}$ lattice of ring resonators can be described by the Coupled Mode Theory (CMT) where the single modes of the ring resonators are coupled by the coupling rate $J$. Furthermore, in this derivation, we have used $\beta L_{\mathrm{LR}}=\beta L_{\mathrm{SR}}+\pi$. Because of this condition, the Hamiltonian does not contain any terms relating to the fields in the link rings. For any given value of $\beta L_{\mathrm{LR}}$, we can still derive the same dispersion relation but with a modified coupling rate $J$ (see Appendix B). However, there is no magnetic field in 


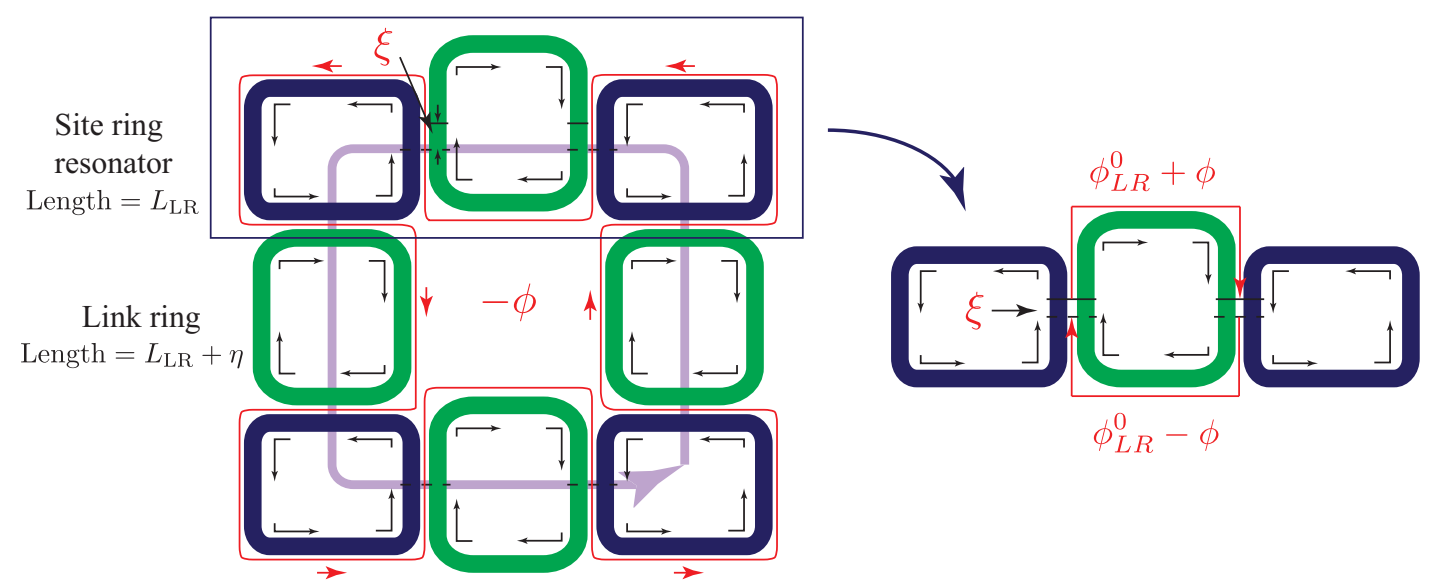

Figure 4.2: A plaquette, composed of 4 site rings and 4 link rings, which introduces synthetic magnetic field for photons by vertically shifting the link ring in the top row of rings. The black arrows in the rings indicates the circulation of photons in the rings and the red arrows highlights the propagation direction around the plaquette. The total phase acquired in going around the loop is $-\phi$ - the magnetic flux. $\phi_{0}^{\mathrm{LR}}=i \beta L_{\mathrm{LR}} / 2$ is the phase acquired in half length of the link ring, without a shift.

this Hamiltonian. We now introduce a synthetic magnetic field for photons in the system.

\subsubsection{Magnetic Field for Photons}

In Chapter 2, we showed that the effect of a magnetic field on a $2 \mathrm{D}$ electron gas is evident in the noncommutative magnetic translation operators. Therefore, for photons travelling in a closed loop, introduction of a phase which depends on the direction of travel around the loop, is equivalent to creating a synthetic magnetic field for photons. In the 2D lattice of ring resonators described above, this can be achieved easily by shifting the link resonators located horizontally between the site rings, in the vertical direction by length $y \xi$, where $y$ is the row index. With this shift, the photons circulating counterclockwise in the site rings, clockwise in the 


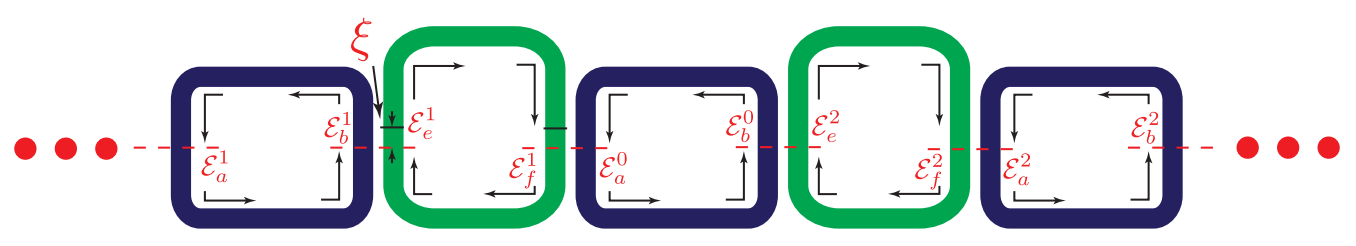

Figure 4.3: An infinite 1D array of site ring resonators (blue), coupled using link rings (green). The link rings are vertically shifted to introduce a direction dependent hopping phase in the Hamiltonian.

link rings, and hopping from left to right travel a slightly longer path and hence accumulate an extra phase when compared to the photons hopping from right to left. Thus, in a closed loop, around a plaquette consisting of 4 site ring resonators and 4 link ring resonators (Fig. 4.2), the photons accumulate a phase $\phi$, when circulating clockwise around the loop and $-\phi$, when circulating counterclockwise around the loop. To implement a uniform magnetic field in the lattice, we choose a gauge $A=(y \xi, 0)$ and vertically shift the link rings in each row by $y \xi$, where $y$ is the row index (Fig. 4.4). The Hamiltonian for this $2 \mathrm{D}$ system of coupled ring resonators is now given by $H_{0}=\sum_{x, y} \hat{a}_{x, y}^{\dagger} \hat{a}_{x, y}-J\left(\sum_{x, y} \hat{a}_{x+1, y}^{\dagger} \hat{a}_{x, y} e^{-i 2 \pi \alpha y}+\hat{a}_{x, y}^{\dagger} \hat{a}_{x+1, y} e^{i 2 \pi \alpha y}+\hat{a}_{x, y+1}^{\dagger} \hat{a}_{x, y}+\hat{a}_{x, y}^{\dagger} \hat{a}_{x, y+1}\right)$.

It remains to be proven that vertically displacing the link ring results in direction dependent hopping phase as it appears in the above Hamiltonian. To this end, we consider a simple one-dimensional infinite chain of resonators coupled using link resonators (Fig. 4.3). Each link resonator is shifted as shown in the figure. The Hamiltonian for this system in the tight-binding model is

$$
H_{0}=\sum_{x} \hat{a}_{x}^{\dagger} \hat{a}_{x}-J\left(\sum_{x} \hat{a}_{x+1}^{\dagger} \hat{a}_{x} e^{-i 2 \pi \alpha}+\hat{a}_{x}^{\dagger} \hat{a}_{x+1} e^{i 2 \pi \alpha}\right)
$$


The dispersion relation for this Hamiltonian can be derived using the procedure we followed in Chapter 2 and is given as

$$
\omega=\omega_{0}-2 J \cos \left(K_{x} \Lambda+\phi\right)
$$

where $\phi=2 \pi \alpha$. Now, as we did for the two dimensional system, we label the field amplitudes in this 1D system (see Fig. 4.3) and use the transfer matrix approach to relate the field amplitudes as

$$
\left(\begin{array}{c}
\mathcal{E}_{a}^{0} e^{-i \beta \frac{L_{\mathrm{SR}}}{4}} \\
\mathcal{E}_{b}^{0}
\end{array}\right)=\frac{1}{i \kappa}\left(\begin{array}{cc}
-t & 1 \\
-1 & t
\end{array}\right)\left(\begin{array}{c}
\mathcal{E}_{d}^{1} e^{-i \beta \frac{L_{\mathrm{LR}}}{2}} e^{i \phi} \\
\mathcal{E}_{c}^{1}
\end{array}\right)
$$

and

$$
\left(\begin{array}{c}
\mathcal{E}_{c}^{1} e^{-i \beta \frac{L_{\mathrm{LR}}}{2}} e^{-i \phi} \\
\mathcal{E}_{d}^{1}
\end{array}\right)=\frac{1}{i \kappa}\left(\begin{array}{cc}
-t & 1 \\
-1 & t
\end{array}\right)\left(\begin{array}{c}
\mathcal{E}_{b}^{1} e^{-i \beta \frac{L_{\mathrm{SR}}}{4}} \\
\mathcal{E}_{a}^{1}
\end{array}\right) .
$$

Again, using Bloch's theorem, we can relate the field amplitudes in different rings as

$$
\begin{aligned}
\mathcal{E}_{a}^{1} & =\mathcal{E}_{a}^{0} e^{i K_{x} \Lambda} \\
\mathcal{E}_{b}^{1} & =\mathcal{E}_{b}^{0} e^{i K_{x} \Lambda}
\end{aligned}
$$

Using these in the above equations, we find two homogenous equations in variables $a_{0}, b_{0}$

$$
\left(\begin{array}{cc}
\Phi_{\mathrm{SR}}-\frac{\Lambda_{x}}{k^{2}}\left(t^{2} \Phi_{\mathrm{LR}}-\Phi_{\mathrm{LR}}^{-1}\right) & \frac{\Lambda_{x} t}{k^{2}} \Phi_{\mathrm{SR}}\left(\Phi_{\mathrm{LR}}-\Phi_{\mathrm{LR}}^{-1}\right) \\
\frac{\Lambda_{x} t}{k^{2}}\left(\Phi_{\mathrm{LR}}^{-1}-\Phi_{\mathrm{LR}}\right) & 1+\frac{\Lambda_{x}}{k^{2}} \Phi_{\mathrm{SR}}\left(\Phi_{\mathrm{LR}}-t^{2} \Phi_{\mathrm{LR}}^{-1}\right)
\end{array}\right)\left(\begin{array}{c}
a_{0} \\
b_{0}
\end{array}\right)=0
$$

where now $\Phi_{\mathrm{LR}}=e^{-i \beta \frac{L_{\mathrm{LR}}}{2}} e^{-i \phi}$. These equations have a non-zero solution if the determinant of the coefficient matrix is zero. Solving the above determinant and 
approximating the phase $\phi$, as we did earlier, gives us the dispersion relation

$$
\omega=\omega_{0}-2 J \cos \left(K_{x} \Lambda+\phi\right)
$$

Thus our two-dimensional system of coupled ring resonators, where the link resonators are shifted by $y \xi$, implements a uniform synthetic gauge field for photons and the flux of this synthetic magnetic field per plaquette is $\phi$.

\subsection{A Finite Lattice - Transmission, Delay \& Edge States}

In the above discussion, we considered an infinite system of ring resonators and derived its dispersion relation. Since the lattice was infinite, there were no edge states. For a finite lattice, as with its electronic counterpart, edge states appear naturally. In this section, we investigate a finite lattice of these ring resonators using the coupled-mode theory, i.e., treating the ring resonators in the single-mode approximation, with a coupling between the modes. The lattice is also coupled to input and output waveguides to allow probing of the lattice (similar to an ADF). We use input-output formalism to describe coupling of the lattice to the input and output fields, and relate them using power transmission and delay properties of the lattice [44]. We will show that the presence of edge states is evident in the transmission and delay spectra of the lattice.

We proved that the $2 \mathrm{D}$ lattice of ring resonators is described by the tightbinding Hamiltonian in Eq. 4.17. We use this Hamiltonian along with the inputoutput formalism to express output fields at the drop and through ports in terms of the incoming fields. The Hamiltonian for a lattice of $N_{\mathrm{SR}}=N_{x} \times N_{y}$ site rings 


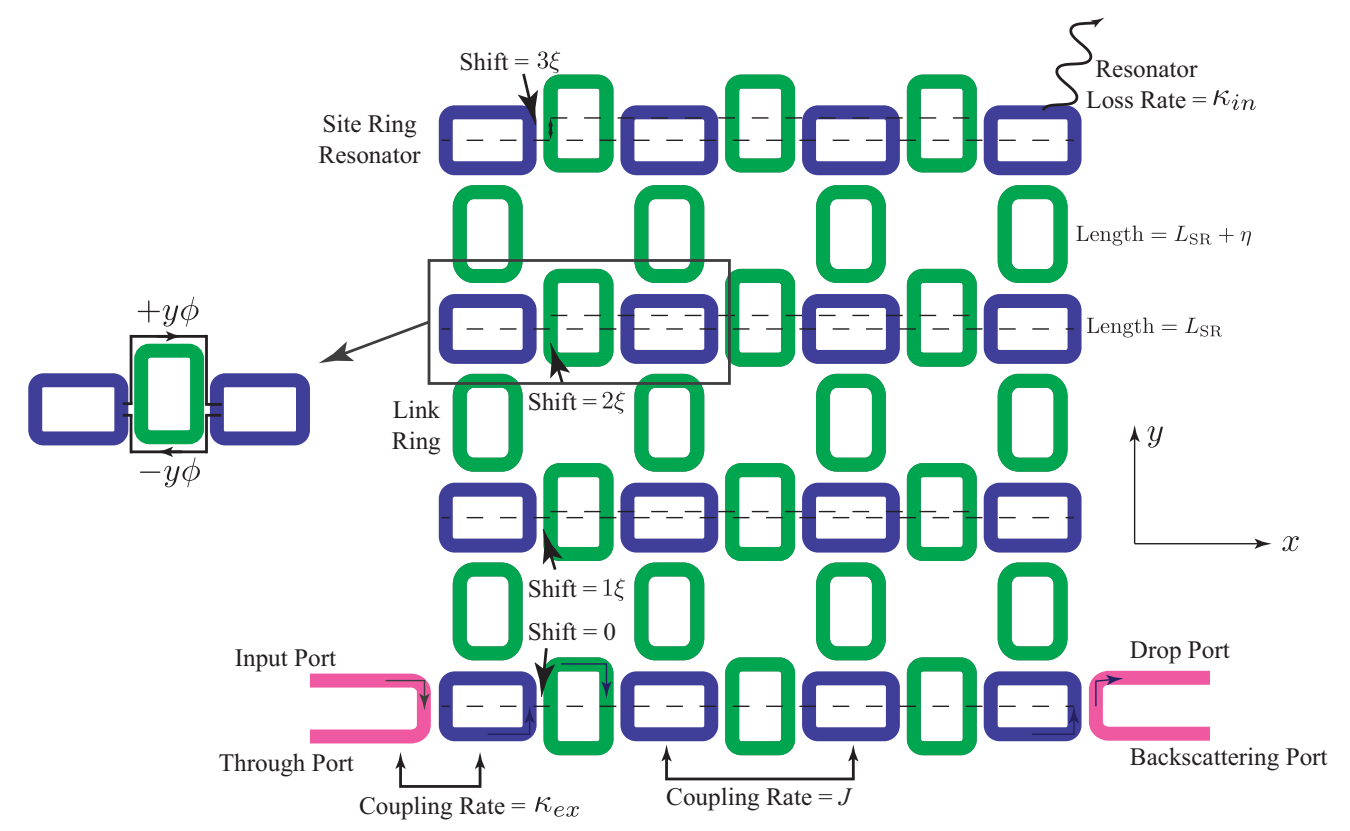

Figure 4.4: A $4 \times 4$ lattice of site rings. The link rings are shifted vertically to introduce a uniform synthetic magnetic field. The lattice is coupled to input and output waveguides to probe the transmission and delay spectra of the lattice

is a square matrix of dimensions $N_{\mathrm{SR}} \times N_{\mathrm{SR}}$. We choose the configuration space itself as the basis - a basis of dimension $N_{\mathrm{SR}}$, with the rings of the lattice indexed linearly. The diagonal elements of the matrix are then the self energy terms, i.e., the resonance frequency of the resonator $\omega_{0}$. The off-diagonal elements of the matrix represent the couplings between rings. For example, $H_{0}^{i, j}=-J e^{ \pm i \alpha y}$ if $\operatorname{ring} i$ is coupled to ring $j$ and both the rings $i, j$ are in the same row, with index $y$. The sign of the phase depends on the hopping direction. For hopping along $y$-axis, there is no phase factor since we choose the Landau gauge. For example, in this basis, the Hamiltonian, $H_{0}$, for a $3 \times 3$ lattice is a square matrix of dimension $9 \times 9$ 


$$
H_{0}=\left(\begin{array}{ccccccccc}
\omega_{0} & -J e^{-i \alpha} & 0 & -J & 0 & 0 & 0 & 0 & 0 \\
-J e^{i \alpha} & \omega_{0} & -J e^{-i \alpha} & 0 & -J & 0 & 0 & 0 & 0 \\
0 & -J e^{i \alpha} & \omega_{0} & 0 & 0 & -J & 0 & 0 & 0 \\
-J & 0 & 0 & \omega_{0} & -J e^{-i 2 \alpha} & 0 & -J & 0 & 0 \\
0 & -J & 0 & -J e^{i 2 \alpha} & \omega_{0} & -J e^{-i 2 \alpha} & 0 & -J & 0 \\
0 & 0 & -J & 0 & -J e^{i 2 \alpha} & \omega_{0} & 0 & 0 & -J \\
0 & 0 & 0 & -J & 0 & 0 & \omega_{0} & -J e^{-i 3 \alpha} & 0 \\
0 & 0 & 0 & 0 & -J & 0 & -J e^{i 3 \alpha} & \omega_{0} & -J e^{-i 3 \alpha} \\
0 & 0 & 0 & 0 & 0 & -J & 0 & -J e^{i 3 \alpha} & \omega_{0}
\end{array}\right) .
$$

The time evolution of the ring energy amplitude operators $\left(\hat{a}_{i}\right)$ are then given by a set of coupled differential equations as

$$
\frac{d \hat{a}_{i}}{d t}=i\left[H, \hat{a}_{i}\right]-\kappa_{\mathrm{in}} \hat{a}_{i}-\left(\delta_{i, \mathrm{I}}+\delta_{i, \mathrm{O}}\right) \kappa_{\mathrm{ex}} \hat{a}_{i}-\delta_{i, \mathrm{I}} \sqrt{2 \kappa_{\mathrm{ex}}} \mathcal{E}_{\mathrm{I}}(t)
$$

The first term on the right gives the resonance frequency term, $\omega_{0}$, and the couplings of the ring $k$ to its neighbors. The second term is the loss rate, $\kappa_{\text {in }}$, of the ring. The third term represents the loss of energy at the input and output ports. Even though it is an input port, it is a waveguide coupler and hence, also acts as an output - we call it the through port. The fourth term is the energy input to the lattice by an electric field $\mathcal{E}_{\mathrm{I}}(t)$. The electric field here is normalized to power, i.e., the square of the electric field amplitude is power and not the energy, as we did in the description of a single ring resonator in Chapter 3. These relations are valid for an electric field excitation with an arbitrary time behavior. When the input electric field is a plane 
wave, of the form $\mathcal{E}_{\mathrm{I}} e^{-i \omega t}$, the above relations can be simplified in the rotating frame to give

$$
-i \omega a_{i}=i\left[H, a_{i}\right]-\kappa_{\text {in }} a_{i}-\left(\delta_{i, \mathrm{I}}+\delta_{i, \mathrm{O}}\right) \kappa_{\mathrm{ex}} a_{i}-\delta_{i, \mathrm{I}} \sqrt{2 \kappa_{\mathrm{ex}}} \mathcal{E}_{\mathrm{I}},
$$

where there is no time dependence now. Also, the ring energy amplitudes $a_{i}$ are now the expectation values of the corresponding operators i.e., $a_{i}=\left\langle\hat{a}_{i}\right\rangle$. The above equations are a set of coupled linear equations and can be solved using matrix methods. The output at the drop and through ports is

$$
\begin{aligned}
& \mathcal{E}_{\mathrm{D}}=\sqrt{2 \kappa_{\mathrm{ex}}} a_{\mathrm{O}} \\
& \mathcal{E}_{\mathrm{T}}=\mathcal{E}_{\mathrm{I}}-\sqrt{2 \kappa_{\mathrm{ex}}} a_{\mathrm{I}},
\end{aligned}
$$

where we have used the single ring relations as given in Eqs. 3.20-3.21.

A typical spectrum at the drop and through ports for a $8 \times 8$ lattice is shown in Fig. 4.5. The magnetic field has been chosen such that $\phi=2 \pi \alpha_{\mathrm{M}}=2 \pi / 4$. Now, if we look at the energy distribution in the lattice we find that the highlighted regions are the edge state bands. Further analysis of the probability current in these regions reveals the direction of circulation of these edge states. We refer the counterclockwise propagating states as the short-edge and the clockwise propagating states as the long-edge, because of the path length they travel through the lattice from input to the output. The bulk states, on the other hand, show a random distribution and a random probability current. Also, the edge-state distribution remains the same even when the input frequency is changed by $\approx 0.5 \mathrm{~J}$, whereas, the bulk state distribution changes for even $\approx 0.01 J$ change in the input frequency.

Fig. 4.5(b) shows the delay spectrum at the drop port for this lattice. The 

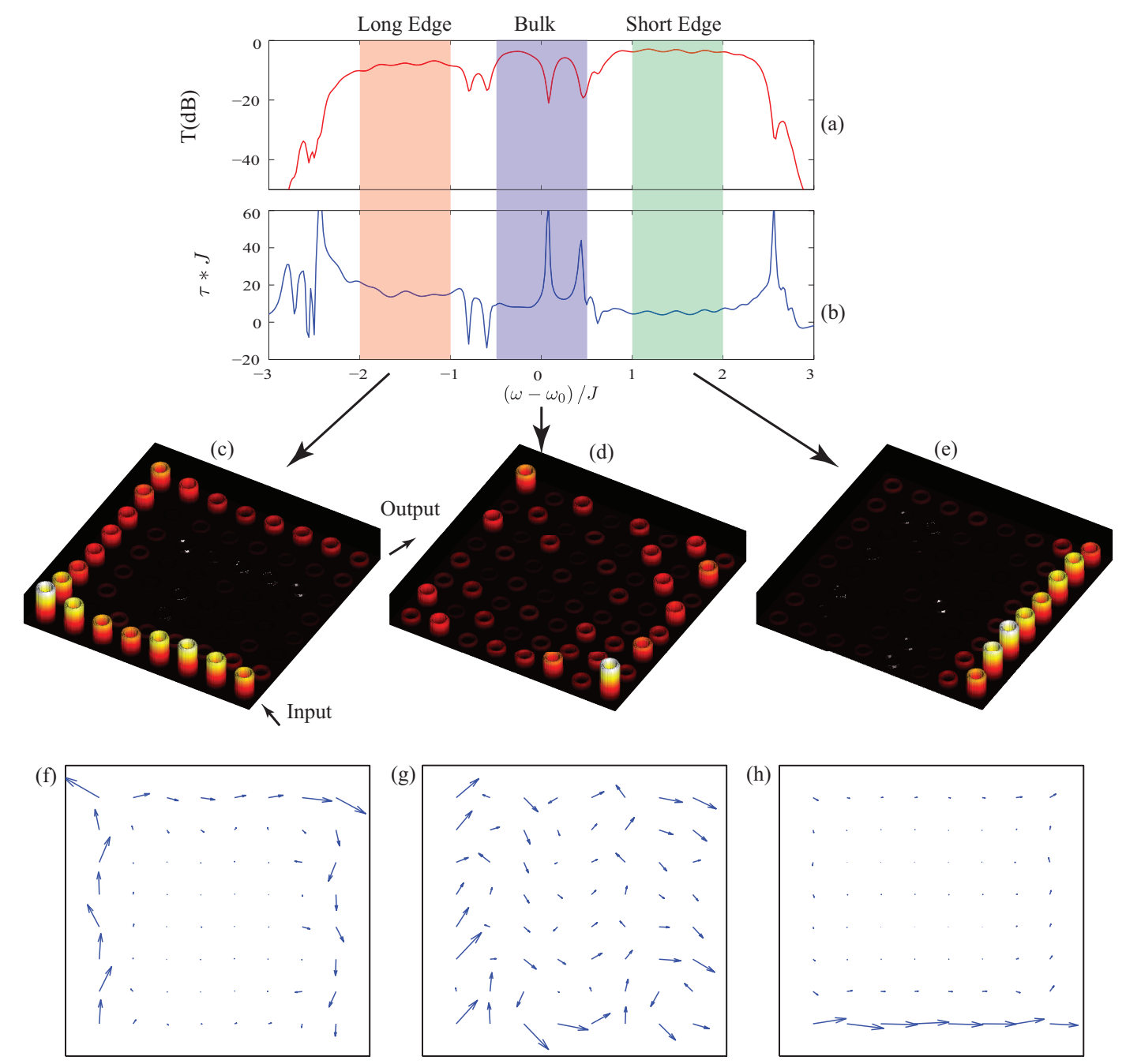

Figure 4.5: (a) A typical transmission and (b) delay spectrum for an $8 \times 8$ lattice. The long-edge band (red), bulk-band (blue) and the short-edge band (green) are highlighted. (c-e) Ring intensity distributions for the edge and the bulk states. The short-edge and the long-edge paths can be easily identified. The bulk-state distribution is random. (f-h) Probability currents for the edge states in (c-e). The short and the long-edge states have opposite currents. Here we have used $\kappa_{\text {ex }}=$ $1.25 \mathrm{~J}$ and $\kappa_{\text {in }}=0.08 \mathrm{~J}$. 

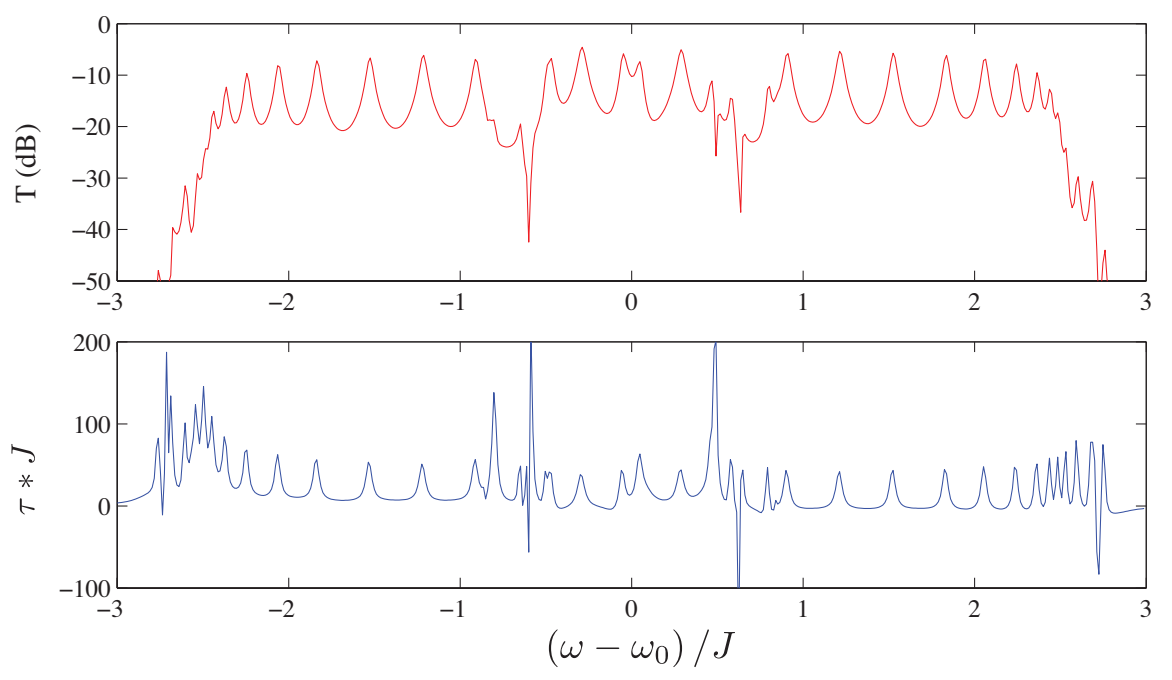

Figure 4.6: Transmission spectrum of a $8 \times 8$ lattice with weak input/output coupling, $\kappa_{\mathrm{ex}}=0.1 \mathrm{~J}$. Individual edge states can now be resolved.

delay shown here is the Wigner delay which is calculated using the derivative of the phase of the electric field at drop port as

$$
\tau_{\mathrm{D}}=\frac{d}{d \omega}\left(\frac{\mathcal{E}_{\mathrm{D}}}{\left|\mathcal{E}_{\mathrm{D}}\right|}\right)
$$

We see that the delay is flat in regions of edge states. We also observe that the delay in the long-edge state is higher than the delay in short-edge state since the former travels a longer path. We should note that the Wigner delay can be negative for anomalous dispersion regions around a phase jump, as is evident in the delay spectrum.

We discussed in Chapter 2 that the Hofstadter spectrum for a finite lattice exhibits discrete edge states in the bandgap. However, the individual edge state peaks are not resolved in this spectrum. This is a result of a strong coupling $\kappa_{\text {ex }}$ to input and output waveguides used for the plots in Fig. 4.5 and also the high intrinsic loss $\kappa_{\text {in }}$, which broadens the individual peaks. Fig. 4.6 shows a spectrum for the 


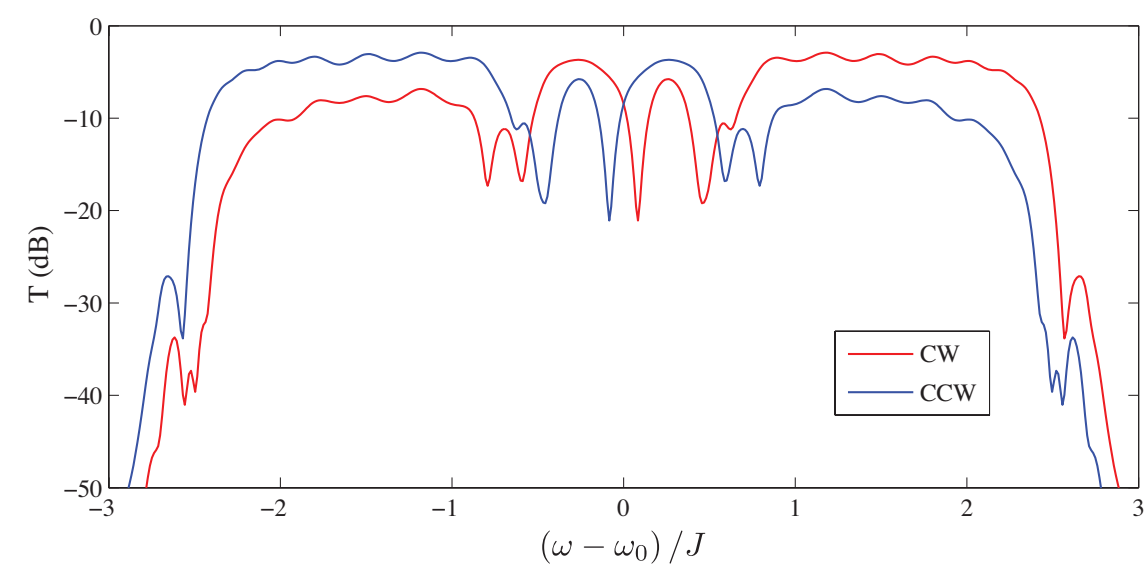

Figure 4.7: Flipping of the transmission spectrum as a result of spin reversal. Edge state currents also get reversed.

same device where now $\kappa_{\mathrm{ex}}=0.1 J$ and $\kappa_{\text {in }}=0.01 J$. In this regime, the spectrum clearly shows transmission through individual edge states and it corresponds to a cross-section of the Hofstadter butterfly spectrum, with finite lattice size, at $\alpha=$ 1/4. When the input field frequency coincides with one of the eigenstates, the transmission is high (and low otherwise). Also, since we couple light to a single ring in the lattice, a point-like excitation, we can excite all the eigenstates of the system.

\subsection{Spin Degree of Freedom}

In the preceding discussion, the field circulated counterclockwise in the site resonators and clockwise in the link resonators. However, a ring resonator can support two degenerate clockwise and counterclockwise propagating modes. Therefore, with this $2 \mathrm{D}$ system of coupled ring resonators, the site resonators support a counterclockwise circulating mode and the link resonators support a clockwise circulating mode or vice-versa. This is equivalent to having a spin degree of freedom in the 
lattice with two possible states - \pm 1 . Furthermore, because of their opposite sense of rotation in the rings, they also experience opposite magnetic fields (sign reversed magnetic flux) in the lattice. The Hamiltonian for this system considering the spin degree of freedom is thus given as

$$
\begin{aligned}
H_{0} & =\sum_{\sigma, x, y} \hat{a}_{\sigma, x, y}^{\dagger} \hat{a}_{\sigma, x, y} \\
& -J\left(\sum_{\sigma, x, y} \hat{a}_{\sigma, x+1, y}^{\dagger} \hat{a}_{\sigma, x, y} e^{-i 2 \pi \alpha \sigma}+\hat{a}_{\sigma, x, y}^{\dagger} \hat{a}_{\sigma, x+1, y} e^{i 2 \pi \alpha \sigma y}+\hat{a}_{\sigma, x, y+1}^{\dagger} \hat{a}_{\sigma, x, y}+\hat{a}_{\sigma, x, y}^{\dagger} \hat{a}_{\sigma, x, y+1}\right),
\end{aligned}
$$

where $\sigma$ is \pm 1 , representing the spin. Also, the sign of hopping phase in this Hamiltonian is now spin dependent, indicating reversal of magnetic field. The Hamiltonian does not contain a term which couples these spin states. However, backscattering in the ring waveguides can lead to a mixing of the two states. But as we will show in Chapter 5, this coupling is negligible.

For a given input frequency, the spin reversed states also rotate in opposite direction around the lattice, i.e., the edge state currents are reversed. As shown in Fig. 4.7, with spin reversal, the long-edge and the short-edge states have also reversed positions in the spectrum. Our system is therefore equivalent to a quantum spin-Hall system where the two degenerate spin states travel in opposite directions $[46,47]$. In the finite lattice (Fig. 4.4), spin can be easily reversed by launching light into the through port and observing the output at the backscattering port. Coupling light into the drop port does reverse the spin, but it also reverses the direction of magnetic field and hence has no effect on the spectrum. 


\subsection{Edge States as Robust Optical Delay Lines}

Optical delay lines find frequent use in optical telecommunication systems, such as in optical switching, optical header processing, channel interleaving, etc. [48-50]. An ideal optical delay line, integrated on a chip, would achieve maximum delay with minimum loss and have a minimal footprint on the chip. The delay line should also be broadband with operating bandwidth of at least a nm. Conventionally

a linear (1D) array of resonators, called a Coupled Resonator optical Waveguide (CROW), has been proposed as optical delay line [51,52]. A CROW can be realized using ring resonators or using photonic crystal cavities and provides a significant reduction in the device footprint when compared to a simple, long waveguide $[42$, 53-55]. As discussed in Section 4.1, the Hamiltonian for such an infinite 1D array of ring resonators is

$$
H_{0}=\sum_{x} \hat{a}_{x}^{\dagger} \hat{a}_{x}-J\left(\sum_{x} \hat{a}_{x+1}^{\dagger} \hat{a}_{x}+\hat{a}_{x}^{\dagger} \hat{a}_{x+1}\right) .
$$

A finite 1D array of ring resonators with input/output couplings can be analyzed similarly to a 2D lattice. The simulated transmission and delay spectra for an array with 20 rings is shown in Fig.4.8.

For an ideal array of ring resonators, the delay increases linearly with the number of rings in the array, without compromising the bandwidth. The bandwidthdelay product of such an array is therefore a monotonically increasing function of the length of the array. However, integrated photonic device fabrication invariably leads to lattice disorders. Disorder can manifest as a mismatch of the ring resonance fre- 

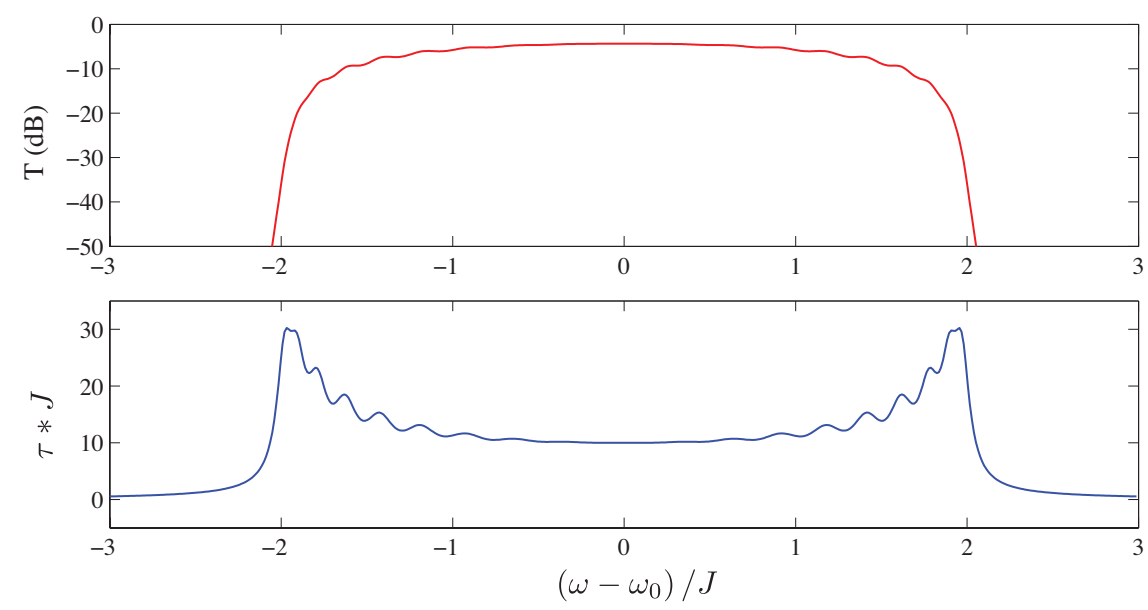

Figure 4.8: Transmission and delay spectrum of a 20 ring array.

(a)

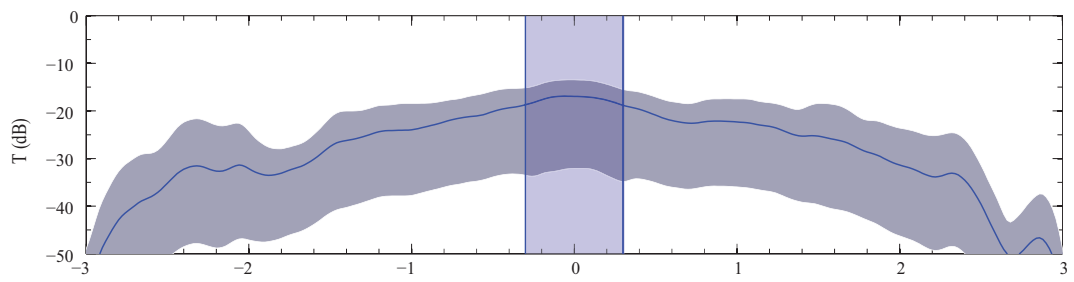

(b)

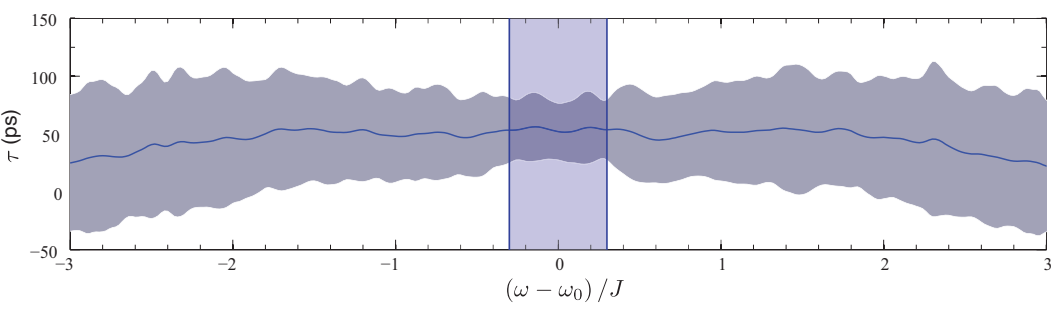

(c)

00000000000000000000

(d)

\section{0}

Figure 4.9: Effect of disorder on the (a) transmission, (b) delay spectrum of the 20 ring array. The solid blue line shows the mean transmission and the grey shading indicates the standard deviation resulting from disorder. (c) Intensity distribution in the array for a pure system, in the mid-band. The intensity distribution extends throughout the length of the array. (d) Intensity distribution in the array for same system, but in the presence of strong disorder. The intensity distribution is localized towards the input of the array. 


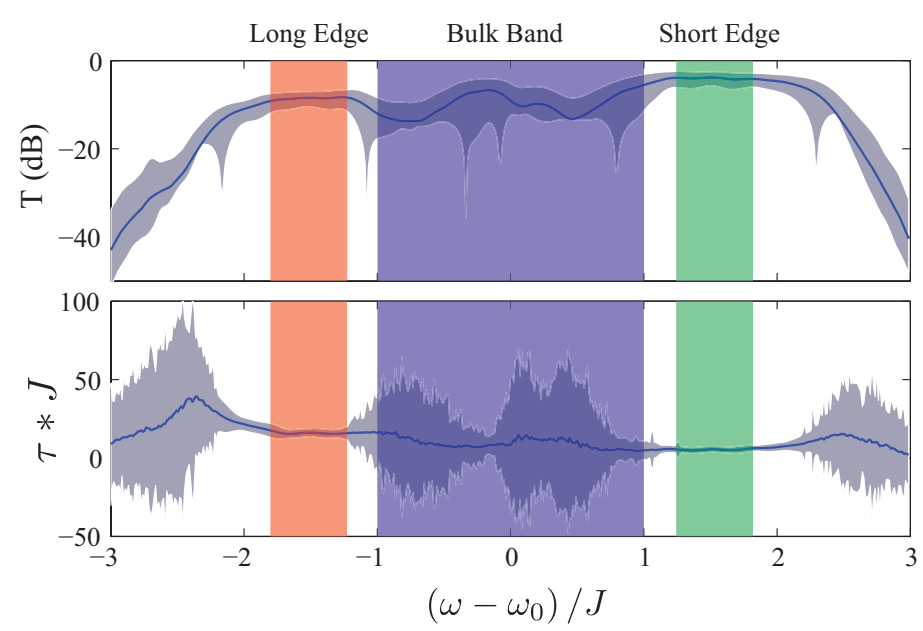

Figure 4.10: A typical transmission and delay spectrum of an $8 \times 8$ lattice. The edge state regions, highlighted, are less susceptible to disorder.

quencies $\Delta \omega_{0}$, variations in the coupling constant $\Delta J$ and the loss rate $\Delta \kappa_{\text {in }}$. In the presence of disorder, the transmission of the array falls exponentially, in addition to the usual reduction in transmission due to resonator losses $[55,56]$. Also, the bandwidth of the delay line decreases as the length of the array increases [57]. Ultimately, as the length of the array or the disorder strength increases, localization of light is observed, i.e., the forward transmission of light is halted (Fig. 4.9) [58-60]. The light does not couple to the lattice and instead appears at the through port. This localization of light is similar to Anderson localization observed in low-dimensional electronic systems and has been observed experimentally [58] in an array of ring resonators. In this regime, the only mode of transmission is tunneling through localized states. Such a transmission is characterized by dips in the transmission spectrum and peaks in the delay spectrum, because of the slow tunneling rate through localized states.

Since the edge states are quasi-one-dimensional, they behave exactly as 1D de- 
lay lines, but with the obvious advantage of being robust against certain fabrication disorders. Fig. 4.10 shows the transmission and delay spectrum of a lattice in the presence of disorders $\left(\Delta \omega_{0}, \Delta J, \Delta \kappa_{\text {in }}\right)$. We clearly see that the edge state regions are much less susceptible to disorder, i.e., their transmission and delay are least affected. Contrary to this, the bulk-state region is very noisy. These edge states are however not robust against all the fabrication disorders, for example, back-scattering (spin reversal in ring resonators) due to waveguide surface roughness. For electronic systems, the real magnetic field breaks the time-reversal symmetry and the edge states are also robust against scattering which couples the clockwise and the counterclockwise spin states. In other words, the clockwise and counterclockwise propagating states have different energies. However, for our photonic analogue of the quantum Hall effect, the synthetic magnetic field does not break the time-reversal symmetry and hence the system is not robust against spin coupling disorders. But, in our photonic system, we can selectively excite a spin state using a specific input waveguide (see Fig. 4.4). Also, as we will see in the next chapter, the use of directional couplers to couple ring resonators ensures that the spin coupling disorder is negligible.

\subsection{Transfer Matrix Simulations}

The single-mode approximation and the coupled-mode theory (CMT) discussed in the previous sections is only valid when the ring resonators are coupled weakly, i.e., when the resonance bandwidth is much less than the FSR of the ring. It is therefore imperative that we compare the CMT results to those simulated using 


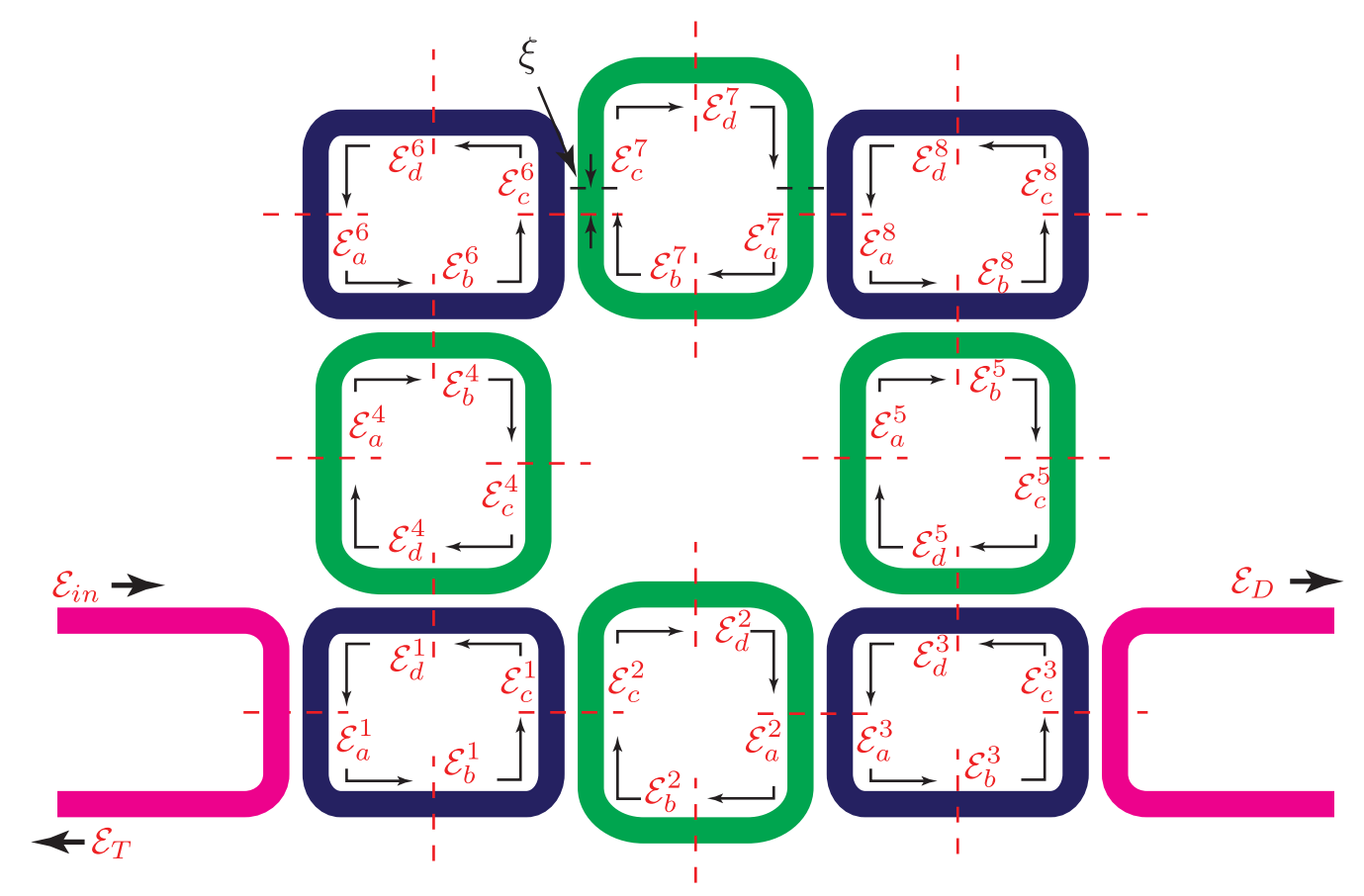

Figure 4.11: A $2 \times 2$ lattice used an example to demonstrate the transfer matrix approach to simulate the transmission and delay spectrum of the lattice.

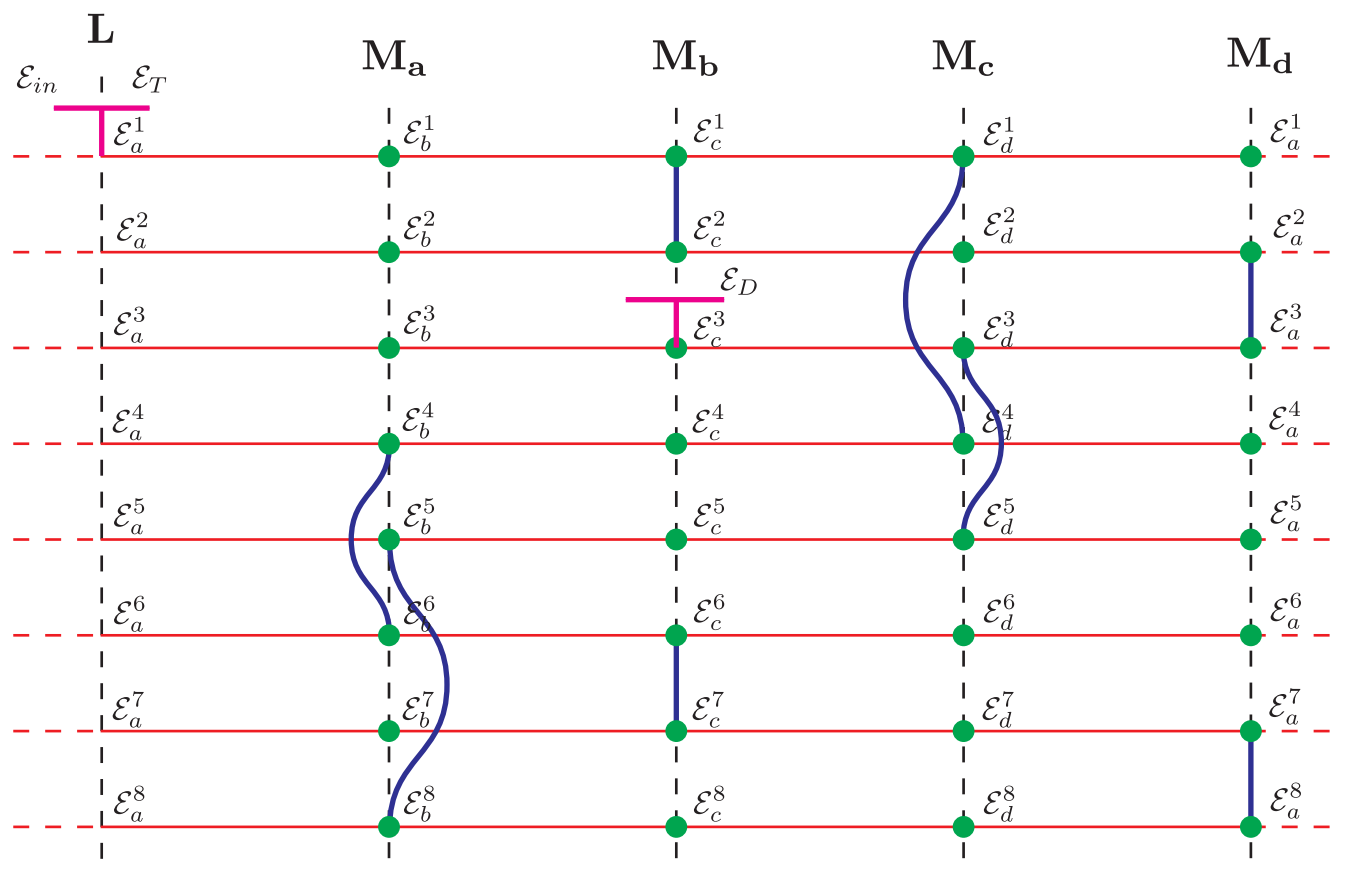

Figure 4.12: Unfolded paths through the $2 \times 2$ lattice. The straight red lines represent propagation in the ring waveguides. The green dots are the coupling regions. The blue lines represent a coupling between two regions. The transfer between two sections is described by matrices $\mathrm{M}_{\mathrm{a}, \mathrm{b}, \mathrm{c}, \mathrm{d}}$. Input and output couplers are also shown. 
the more accurate transfer-matrix approach, where the field solution at any point along the length of the ring can be computed. Further, in this approach, the link rings are not merely represented by a coupling term $J$. The link rings act as resonators, though at a resonance frequency different from the main site rings, because of the difference in ring lengths. This approach, therefore, includes the loss and delay incurred in the travelling through the link resonators.

Since the ring resonators are coupled in two dimensions, it is not easy to write an explicit set of equations relating the field amplitudes inside the rings to the input field amplitude. We have developed a simulation technique following a procedure reported in [61]. As an example of this technique, we consider a $2 \times 2$ lattice and labelling the field amplitudes in the site and link rings as shown in Fig.4.11. The field arrays $\mathcal{E}_{a, b, c, d}$ are such that

$$
\mathcal{E}_{a}=\left[\mathcal{E}_{a}^{1}, \mathcal{E}_{a}^{2}, \mathcal{E}_{a}^{3}, \ldots \mathcal{E}_{a}^{N}\right]^{T}
$$

The length of this vector $N=N_{\mathrm{SR}}+N_{\mathrm{LR}}$, where $N_{\mathrm{SR}}=N_{x} \times N_{y}$ is the number of site rings and $N_{\mathrm{LR}}=\left(N_{y}-1\right)+N_{y}\left(N_{x}-1\right)$ is the number of link rings for a $N_{x} \times N_{y}$ lattice. We index the rings serially as shown in Fig.4.11. Also the field amplitudes are the field values just at the output of a coupling region. We now unfold the rings as straight waveguides, with sections corresponding to propagation between the coupling regions (Fig.4.12). The propagation of field components within each section accumulates a phase as well as attenuation because of waveguide losses. For the site resonators, the accumulated phase and attenuation in each section is $e^{-i \beta \frac{L_{\mathrm{SR}}}{4}} e^{-\alpha \frac{L_{\mathrm{SR}}}{4}}$. For the link resonators, however, the phase and attenuation will 
correspond to half-length of the link ring and will also depend on the location of the link ring so that the vertical shifting of rings is included.

The field amplitudes at the section endpoints are now coupled to other waveguides (representing other rings), according to the coupling pattern in the square lattice. Each coupling region, shown by blue lines, is represented by a transfer matrix

$$
\left(\begin{array}{cc}
t & i \kappa \\
i \kappa & t
\end{array}\right)
$$

which gives the outgoing fields of the coupler as a function of the incoming fields. The complete transfer matrix for the lattice is thus of dimension $N$. For example, the matrix $\mathbf{M}_{a}$ describing the intersection between sections $a$ and $b$ is

$\mathbf{M}_{a}=\left(\begin{array}{cccccccc}\Phi_{\mathrm{SR}} A_{\mathrm{SR}} & 0 & 0 & 0 & 0 & 0 & 0 & 0 \\ 0 & \Phi_{\mathrm{LR}} A_{\mathrm{LR}} & 0 & 0 & 0 & 0 & 0 & 0 \\ 0 & 0 & \Phi_{\mathrm{SR}} A_{\mathrm{SR}} & 0 & 0 & 0 & 0 & 0 \\ 0 & 0 & 0 & t \Phi_{\mathrm{LR}} A_{\mathrm{LR}} & 0 & i k & 0 & 0 \\ 0 & 0 & 0 & 0 & t \Phi_{\mathrm{LR}} A_{\mathrm{LR}} & 0 & 0 & i k \\ 0 & 0 & 0 & i k & 0 & t \Phi_{\mathrm{SR}} A_{\mathrm{SR}} & 0 & 0 \\ 0 & 0 & 0 & 0 & 0 & 0 & \Phi_{\mathrm{LR}} \Phi_{M} A_{\mathrm{LR}} & 0 \\ 0 & 0 & 0 & 0 & i k & 0 & 0 & t \Phi_{\mathrm{SR}} A_{\mathrm{SR}}\end{array}\right)$

Here $\Phi_{\mathrm{SR}}=e^{-i \beta L_{\mathrm{SR}} / 4}$ and $\Phi_{\mathrm{LR}}=e^{-i \beta L_{\mathrm{LR}} / 2}$ are the propagation phase terms. Similarly, $A_{\mathrm{SR}}=e^{-\alpha L_{\mathrm{SR}} / 4}$ and $A_{\mathrm{LR}}=e^{-\alpha L_{\mathrm{LR}} / 2}$ are the attenuation terms. The phase $\Phi_{M}=e^{i \beta \xi}$ arises because of the vertical shift of the link resonator (ring number 7 ). 
We can write similar matrices, $\mathbf{M}_{b, c, d}$ for the other coupling regions as well. Then,

$$
\mathcal{E}_{a}=\mathbf{L M} \mathcal{E}_{a}+\mathcal{E}_{\mathrm{I}}
$$

where $\mathbf{M}=\mathbf{M}_{d} \mathbf{M}_{c} \mathbf{M}_{b} \mathbf{M}_{a}$ describes the complete evolution of the field in the rings. The matrix $\mathbf{L}$ represents the coupling of the lattice to the input and output waveguides, i.e., the drop and the through ports

$$
\mathbf{L}=\operatorname{diag}\left[t_{\mathrm{I}}, 0, t_{\mathrm{O}}, 0,0, \ldots 0\right]
$$

The input field array $\mathcal{E}_{\mathrm{I}}$ in this example is

$$
\mathcal{E}_{\mathrm{I}}=\left[i \kappa_{\mathrm{I}} \mathcal{E}_{\mathrm{I}}, 0,0, \ldots, 0\right]^{T}
$$

Here $t_{\mathrm{I}, \mathrm{O}} a n d \kappa_{I, O}$ are the transmission and coupling coefficient for the input and output waveguide couplers to the lattice. Using above equations, we can write the solution of the fields as

$$
\mathcal{E}_{a}=\frac{\mathcal{E}_{\mathrm{I}}}{(\mathbf{I}-\mathbf{L M})}
$$

where $\mathbf{I}$ is the identity matrix.

Fig. 4.13 shows the transmission and delay spectra for an $8 \times 8$ lattice computed using the transfer matrix method. Also plotted is the transmission spectrum simulated using the CMT. For this simulation, we have used the experimentally observed values for $J, \kappa_{\text {ex }}$ and $\kappa_{\text {in }}$. We can clearly see the agreement between transmission spectra. The small difference in the transmission and delay is because of the the link rings which were absent in the CMT. Field propagation through the link rings results in lower transmission and higher delay for the TMM. The difference is more evident in the long-edge region. 

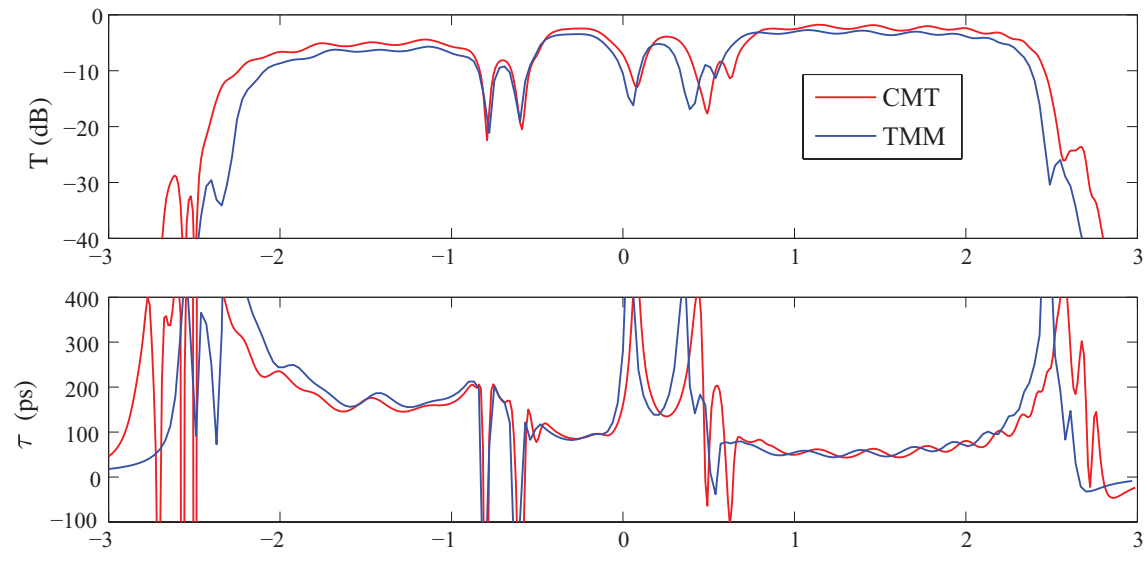

$\left(\omega-\omega_{0}\right) / J$

Figure 4.13: Comparison of the transmission and delay spectra calculated using the TMM and the CMT, for $8 \times 8$ lattice.

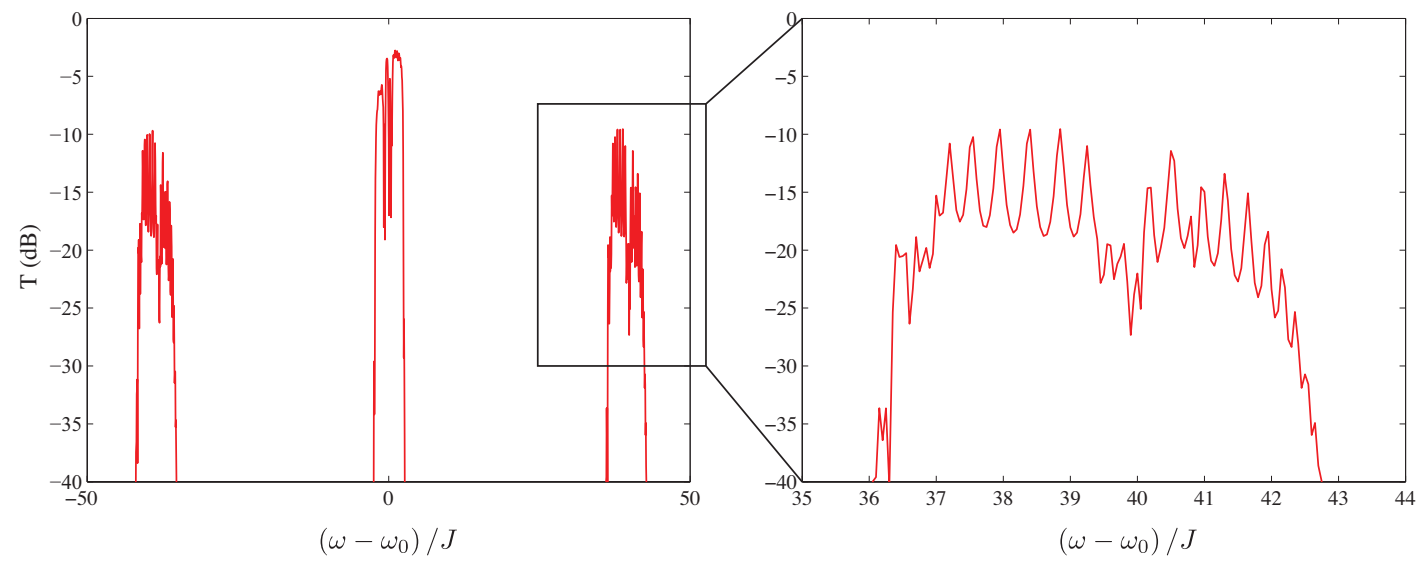

Figure 4.14: Transmission spectrum for the link rings, at the resonance frequency of the link rings. This spectrum is very different from the spectrum of site rings in Fig. 4.13 
Moreover, as we have seen earlier for the case of an ADF, the TMM approach gives the transmission spectrum for the link rings as well (see Fig.4.14). The transmission spectrum for the link rings is significantly different from that of the site rings.

\subsection{Summary}

In this chapter, we discussed, how a 2D lattice of coupled ring resonators simulates a magnetic field for photons. Using dispersion relations, we proved that in the limit of weak coupling, this lattice is defined by the same tight-binding Hamiltonian which describes the electronic quantum Hall system. We then discussed a finite lattice coupled to input and output waveguides and related the fields at the input and output ports of this finite lattice using transmission and delay spectra. We saw that these spectra arise from transmission through edge and bulk states. We explored the spin degree of freedom in this system. With the two spin states of the system, the 2D lattice actually implements the quantum spin-Hall effect. We further discussed an application of the robust edge states as robust optical delay lines. In the last section, we compared the transmission and delay spectrum simulated using the transfer matrix approach to the coupled mode analysis and verified that they are in agreement. 


\section{Chapter 5: Robust Transport of Photons through Edge States}

In this chapter, we present the experimental realization of the system of coupled ring resonators which implements a synthetic magnetic field for photons and hence demonstrates topologically robust edge states. We use the versatile silicon photonics platform to fabricate our devices. We start with a discussion of the device fabrication, followed by device design and the experimental setup to measure the transmission and delay spectrum of the devices. We demonstrate the presence of edge states in our system using direct imaging. We further analyze the transmission and delay distribution statistics made over a number of devices and quantitatively establish the robustness of edge states.

\subsection{Device Fabrication}

A variety of materials like silicon, silicon-nitride, doped silica, lithium-niobate, GaAs, etc. have been widely used to implement ring waveguide resonators [62-66]. Of these available choice of materials, silicon has the advantages of a particularly high refractive index and a mature, commercially available fabrication technology being used for electronic integrated devices. Silicon waveguides have been commonly fabricated using the silicon-on-insulator (SOI) platform, where a 100-220 nm 


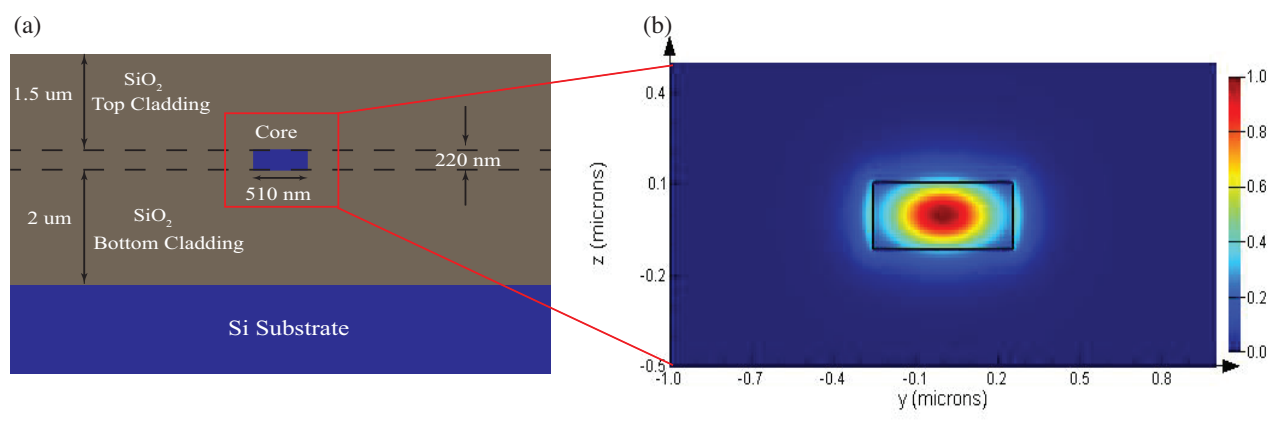

Figure 5.1: (a) Silicon waveguide cross-section used in the ring resonators. (b) Simulated TE mode profile for the waveguide. The waveguide supports single TE mode.

device layer silicon sits on a 2-3 $\mu$ m layer of silicon dioxide grown over a silicon substrate [63]. The $\mathrm{SiO}_{2}$ buffer acts as the bottom cladding layer and also provides optical isolation of the device layer silicon from the bulk silicon substrate. To reduce waveguide scattering loss, a top cladding of $\mathrm{SiO}_{2}$ or PMMA is also used. A schematic of the typical silicon waveguide cross-section is shown in Fig. 5.1(a).

Fig. 5.1(b) shows the TE mode distribution in the silicon waveguide, simulated using a commercial FDTD mode solver (MODE Solutions from Lumerical). Simulation results indicate that a waveguide of width $510 \mathrm{~nm}$ and height $220 \mathrm{~nm}$ is essentially a single-mode waveguide. The waveguide does support a leaky TM mode but it does not propagate and radiates away after a few bends [67]. Because of its high refractive index, silicon waveguides achieve tight confinement of the travelling mode, low scattering loss and a very small bend radius $[67,68]$. Similar waveguide geometries with widths in the range 450-500 nm and heights of 200-220 $\mathrm{nm}$, have been reported with transmission losses of $\approx 3 \mathrm{~dB} / \mathrm{cm}[67,69]$ and bend radii as small as $2 \mu \mathrm{m}$ with a loss of $0.01 \mathrm{~dB}$ per $90^{\circ}$ turn [67]. The waveguide loss results mainly because of light scattering from surface roughness introduced during the fabrication 

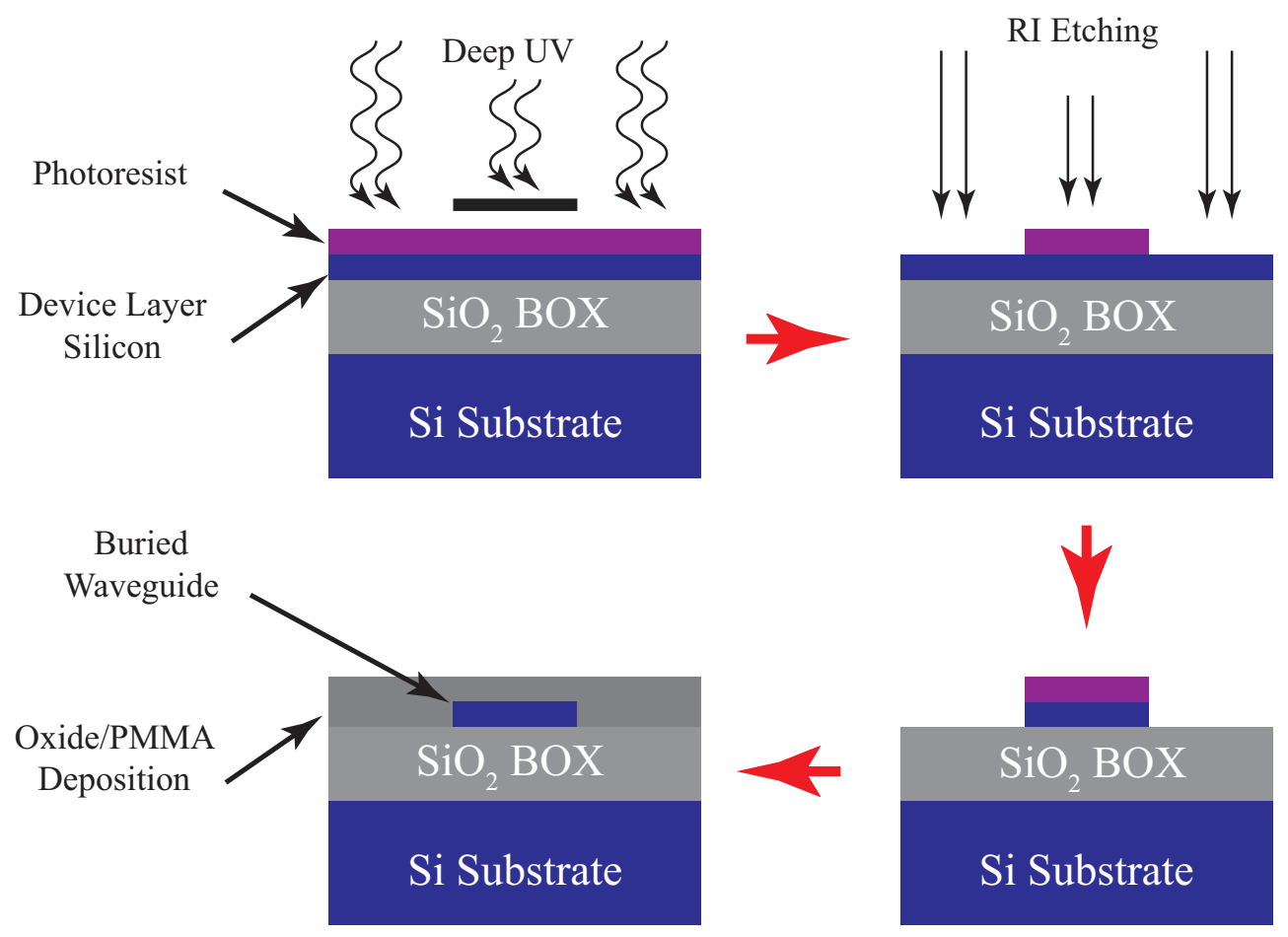

Figure 5.2: A typical photolithography and etching step in the fabrication process. (a) Patterning the photoresist with deep UV light and a mask, (b) subsequent etching of the device layer silicon using reactive ions. (c) The waveguide features emerge after removing the photoresist. (d) Top oxide/PMMA deposition.

process. The absorption loss in silicon is negligible since the photon energy at telecom wavelengths is less than the bandgap of silicon. Silicon is, therefore, a natural choice of material for the design of our ring resonator devices.

Using mode solver, we also estimate $n_{\mathrm{eff}}$ and $n_{\mathrm{g}}$ for the fundamental mode to be 2.46 and 4.07, respectively, at $1550 \mathrm{~nm}$. The large difference between the effective and group indices indicates the significance of dispersion in these waveguides.

\subsubsection{Lithography}

Conventional photolithography has been used to fabricate waveguides and ring resonators where the waveguide dimensions are of the order of a few $\mu \mathrm{m}$. However, 
conventional photolithography can not be used for our system of coupled resonators because it can not provide the resolution required for feature dimensions as small as $\approx 150 \mathrm{~nm}$ which, we will see later, is the evanescent coupling gap between the rings. E-beam lithography is one option which can be used to pattern nanoscale features and has been widely used to fabricate single ring devices [63,66,70]. But it is plagued by the issue of stitching errors $[71,72]$. E-beam lithography writes a pattern in blocks of size 50-100 $\mu \mathrm{m}$ which leads to discontinuities at the boundaries of each block. Because our device structure will be $\approx 500 \mu \mathrm{m} \times 500 \mu \mathrm{m}$, there will be multiple discontinuities in the lattice which will add to disorder. The issue of stitching error has been addressed using Spatially Phase Locked E-Beam Lithography (SPLEBL) but it is hardware intensive and not available commercially [73]. Further, since the e-beam patterns are written serially and our structure is comparatively large, beam parameters (like focus, alignment) can change over the write duration which will result in additional disorder in the devices $[71,72]$.

The best solution then is to use deep UV projection photolithography. It uses light with a wavelength of $193 \mathrm{~nm}$ and can achieve resolution of the order of 130 $\mathrm{nm}$ [69]. The fabrication process starts with patterning the top most silicon device layer using deep UV projection photolithography (Fig. 5.2). Following patterning, the devices are etched using an inductively-coupled plasma etcher. The patterning and etching are done in two steps of $70 \mathrm{~nm}$ and $220 \mathrm{~nm}$ etch depths for the grating couplers and waveguide, respectively. Following etching, the top silicon layer is thermally oxidized to grow a very thin layer of $\mathrm{SiO}_{2}$ and then wet etched to reduce surface roughness. Finally, a layer of silicon oxide $(1.5 \mu \mathrm{m})$ or PMMA $(5 \mu \mathrm{m})$ is 


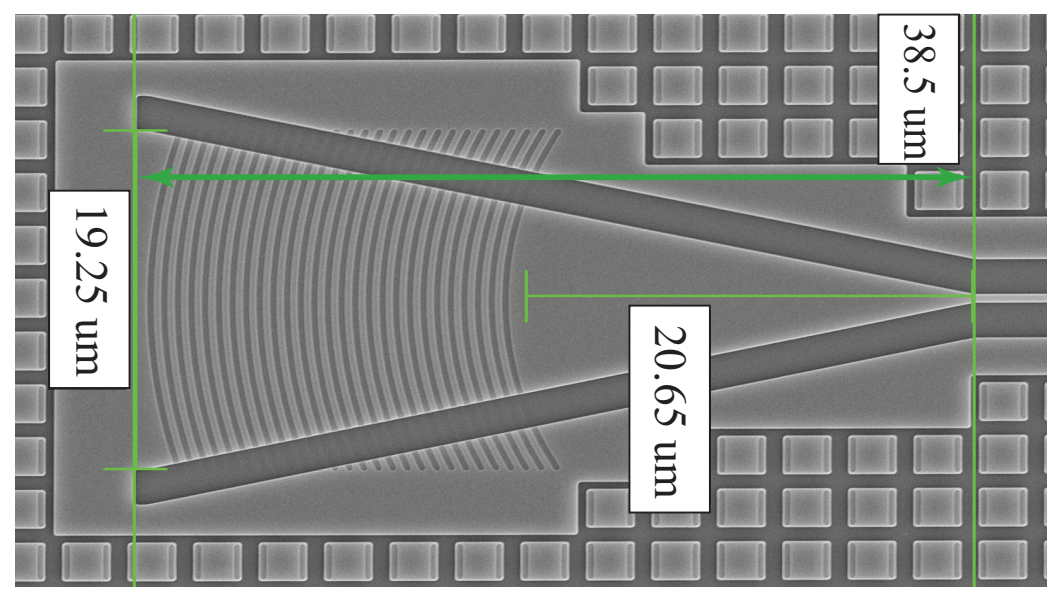

Figure 5.3: SEM of a grating coupler used to couple light to the devices. When light from a standard optical fiber shines on the grating, the dominant diffraction mode travels towards the waveguide. The curved grating helps to focus the fiber mode to the size of the waveguide mode for efficient coupling.

deposited on the top to protect the device surface. This layer also acts as the top cladding for the waveguide.

The fabrication procedure outlined here is compatible with standard CMOS fabrication and hence can be carried out at various commercial foundaries. These devices were fabricated at IMEC foundary in Belgium and LETI in Netherlands.

\subsubsection{Coupling Light to Waveguides}

A typical SOI waveguide has a mode field size of $\approx 500 \mathrm{~nm}$ (see Fig. 5.1) whereas that of a standard optical fiber is $\approx 10 \mu \mathrm{m}$. This large mismatch in the size of the mode field makes direct coupling of light from an optical fiber to the SOI waveguide impossible. An adiabatic, on chip, mode conversion is possible using a tapered waveguide matching the mode of the fiber at input and of the waveguide at output. However, the taper length required to avoid coupling to radiation modes is on the order of a few $\mathrm{mm}[74]$. Very short length $(\approx \mu \mathrm{m})$ inverse tapers with a 
waveguide cross-section of only $\approx 50-100 \mathrm{~nm}$ facing the optical fiber have also been used to couple light into a SOI waveguide [75]. Inverse tapers have been reported with a coupling efficiency as high as $2.7 \mathrm{~dB}[76]$. However, the very small tip size of the inverse taper necessitates the use of e-beam lithography for their fabrication. Moreover, edge coupling to the inverse taper is difficult to achieve because of small misalignment tolerance.

To couple light into the waveguide, we use focussing grating couplers [77-79]. The grating couplers are designed such that when an optical fiber is held at an angle $\left(10^{\circ}\right)$ from the vertical, the dominant diffraction mode of the grating is launched towards the waveguide (Fig. 5.3). Further, the grating is designed as a triangular shaped lens which can focus the fiber mode field $(\approx 10 \mu \mathrm{m}$ in diameter $)$ to the waveguide mode $(\approx 510 \mathrm{~nm})[77]$. These grating couplers have a very small footprint $(40 \mu \mathrm{m} \times 20 \mu \mathrm{m})$ and have an excellent alignment tolerance, with the best reported coupling efficiency of $4 \mathrm{~dB}$ (although with Bragg reflecting mirrors on the bottom, the reported efficiency is as high as $1.6 \mathrm{~dB}$ [79]). Furthermore, the grating couplers are available as standard library elements in IMEC and LETI foundaries and their design has been optimized to achieve maximum coupling efficiency.

\subsection{Device Design and Spectrum Measurements}

As discussed in Chapter 4, the response of the lattice and the presence of edge states can be probed by measuring the transmission and delay spectra of the devices. We use an Optical Vector Analyzer (OVA) to measure the transmission and delay 


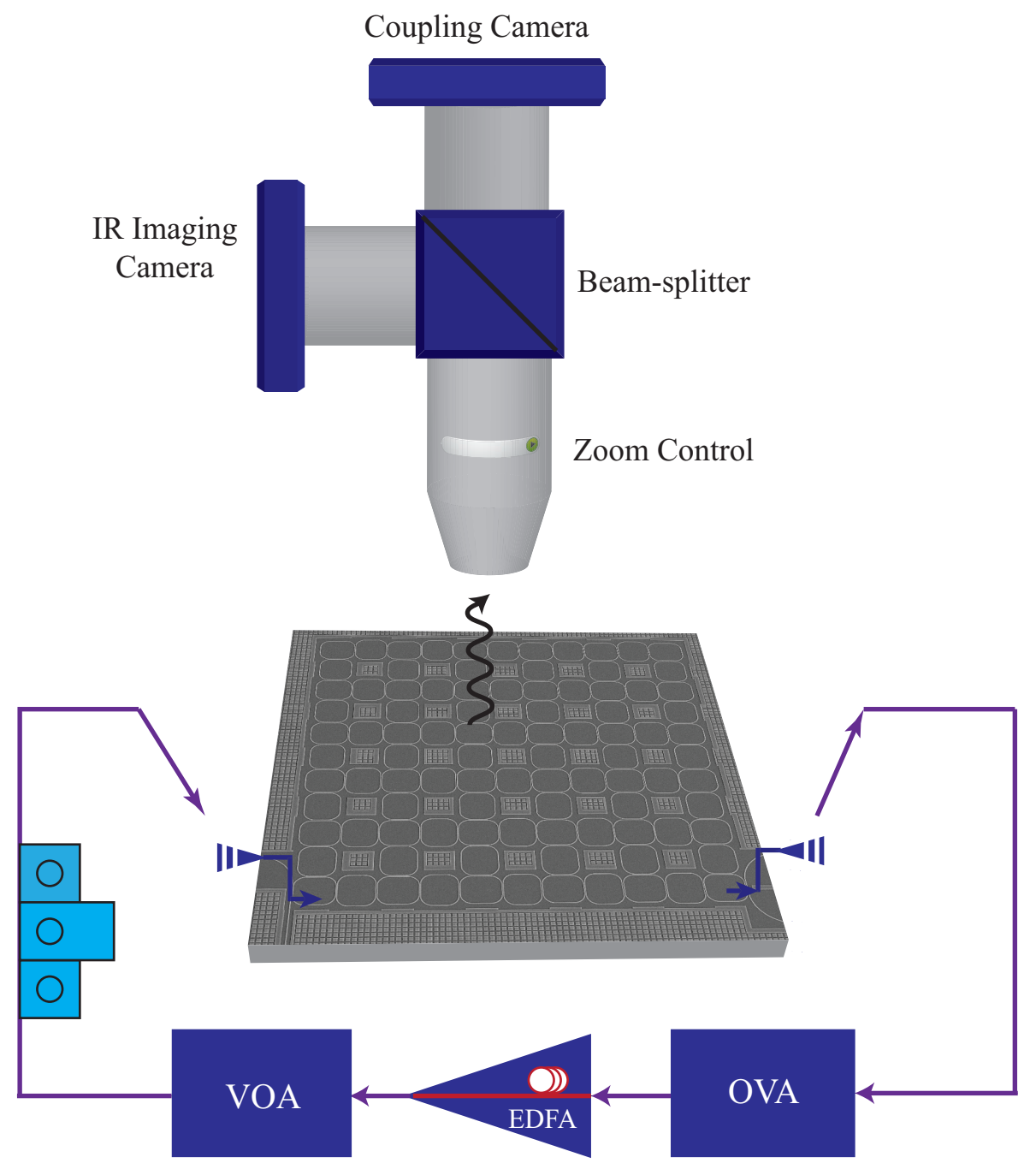

Figure 5.4: Experimental setup for transmission, delay and IR intensity measurements. Light from an optical vector analyzer (OVA) is amplified using an erbiumdoped fiber amplifier (EDFA) and coupled to the lattice using grating couplers. We use a variable attenuator (VOA) to control input power and also a polarization controller to maximize TE polarization being input to the lattice. The microscope objective and the visible CCD camera help to align optical fiber to the grating coupler. The scattered IR light from the lattice is collected by the same objective and imaged on the IR CCD camera, yielding the ring intensity distribution in the lattice. 
spectra. This commercially available OVA from LUNA (OVA5000) is based on swept wavelength interferometry [80]. The analyzer uses a gas-cell-calibrated tunable laser source along with a Mach-Zender interferometer to calculate the complete Jones matrix for the device transmission - the four elements of the matrix along with the relative phase of the elements with respect to the input. These matrix elements are then used to calculate device insertion loss and the accumulated phase for each wavelength. The delay incurred during propagation is calculated as derivative of the phase with respect to angular frequency. In addition, we use an EDFA and a variable optical attenuator (VOA) to control the laser power input to the device. We also use a polarization controller to control the polarization state of the light input to the device. To aid fiber coupling to the grating couplers, we use a microscope objective $(\times 10)$ positioned above the device, along with a CCD camera.

\subsubsection{The ADF Design and Spectrum}

The scanning electron microscope (SEM) image of a typical ADF is shown in Fig. 5.5(a). As is evident, the ring resonator in the ADF is actually in the shape of a racetrack with four straight edges and two of the straight sections of the racetrack are coupled to the input and output waveguides. The coupling rate between the ring waveguide and the input/output waveguide can be expressed in terms of a coupling coefficient between waveguides as

$$
\kappa_{\mathrm{ex}}=\frac{|k|^{2}}{2} \frac{v_{\mathrm{g}}}{L},
$$



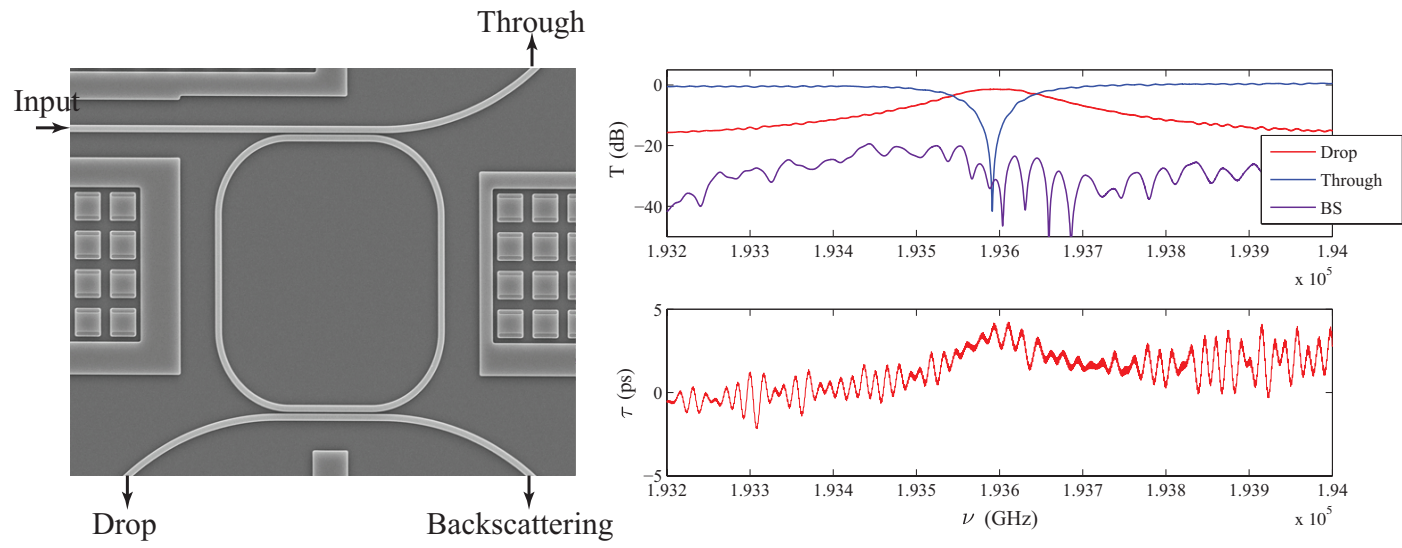

Figure 5.5: (a) SEM image of an ADF. The racetrack resonator is coupled to two waveguides on the top and bottom. The square pattern in the image is to maintain a uniform silicon density across the chip. This helps to achieve a uniform silicon etch rate throughout the chip. (b) Transmission spectrum at the drop, through and backscattering ports. (c) Delay spectrum at the drop port.

where $|k|^{2}$, the cross-coupling coefficient between the waveguides, is a function of the gap between the waveguides and the length of interaction. For a circular ring resonator, because the interaction length is very small, a strong coupling between the ring and the input/output waveguide would require reducing the gap between the waveguides to very small values. This poses a fabrication challenge and also increases the fabrication uncertainty in $\kappa_{\mathrm{ex}}$. The straight sided racetrack resonator thus eases the constraint on coupling gap because of the much longer interaction length in the straight section of the racetrack.

We fix the length of the coupling region to be $7 \mu \mathrm{m}$ and the total racetrack length to be $\approx 70 \mu \mathrm{m}$ in all our devices. Guided by FDTD simulations, we choose a coupling gap of $170 \mathrm{~nm}$ for $\kappa_{\mathrm{ex}}(180 \mathrm{~nm}$ for $J)$ which would result in $\kappa_{\mathrm{ex}} \approx 38 \mathrm{GHz}$. The resulting FSR of $\approx 1000 \mathrm{GHz}(8 \mathrm{~nm})$ is then much larger than the resonance bandwidth of the ADF ( $\approx 140 \mathrm{GHz})$. This choice, thus, also guarantees that the 
coupled-mode theory can be used to simulate the ring resonator devices. The bend radius for the racetrack is chosen to be $6 \mu \mathrm{m}$ so that the bending loss is less than $0.005 \mathrm{~dB}$ per turn [67].

Fig. 5.5(b) shows the experimentally measured transmission spectrum for an ADF using OVA, at the drop, through and backscattering ports. We observe that the output at the backscattering port is $\approx 25 \mathrm{~dB}$ lower than that at the drop port. This suggests that the backscattering due to waveguide surface roughness and directional couplers is negligible. Also shown in Fig. 5.5(c) is the observed delay at the drop port. The maximum delay at the drop port is $3.5 \mathrm{ps}$ and the estimated round trip time is 1 ps. This ring resonator therefore has a very low $Q$ of about 1500 . But this is the loaded $Q$ factor, i.e., it includes the loss to through and drop ports. The $Q$ factor of the resonator can be easily increased by making the ring resonator very weakly coupled to the input/output waveguides (reducing $\kappa_{\text {ex }}$ ). However, a large coupling, larger than the disorder in the lattice, is desirable to demonstrate the presence of edge states in the system.

An ADF can also be used to characterize the coupling rate $\kappa_{\mathrm{ex}}$ (or $J$ ) and the loss rate $\kappa_{\text {in }}$ of the ring resonators. Fig. 5.6 shows a fit to the transmission spectra using single-mode approximation. We see that the SMA gives an excellent fit to the spectra for frequencies close to the resonance. Using (3.24) and (3.29), the fitting parameters, $\kappa_{\text {ex }}$ and $\kappa_{\text {in }}$, can be extracted using the bandwidth and contrast of the 


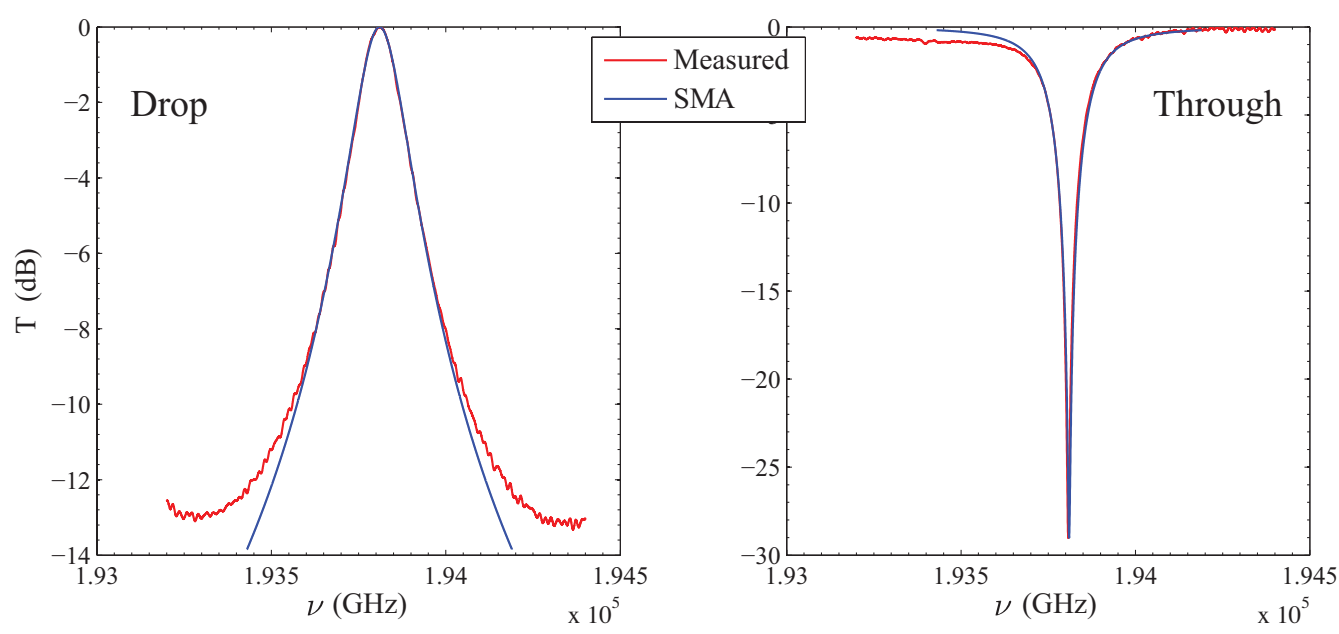

Figure 5.6: A typical transmission spectrum for the ADF, (a) at drop and (b) at through ports. The single-mode approximation fits very well to the measured spectrum for input frequencies close to the resonance, giving $\kappa_{\mathrm{ex}}=38.0 \mathrm{GHz}$ and $\kappa_{\text {in }}=2.9 \mathrm{GHz}$.

transmission spectra as

$$
\begin{aligned}
\kappa_{\mathrm{in}} & =\frac{1}{2} \frac{\mathrm{BW}}{\sqrt{C_{\mathrm{T}}}} \\
\kappa_{\mathrm{ex}} & =\frac{1}{4}\left(\mathrm{BW}-\frac{\mathrm{BW}}{\sqrt{C_{\mathrm{T}}}}\right)=\frac{1}{4}\left(\mathrm{BW}-2 \kappa_{\mathrm{in}}\right),
\end{aligned}
$$

where $C_{\mathrm{T}}$ is the transmission contrast at through port (in linear scale). For the ADF spectra shown in Fig. 5.6, $\kappa_{\mathrm{ex}}=38.0 \mathrm{GHz}$ and $\kappa_{\text {in }}=2.9 \mathrm{GHz}$. The corresponding cross-coupling coefficient $k=0.48$ and the absorption coefficient $\alpha=15.8 \mathrm{~m}^{-1}$. These values are the typical coupling and loss rates in our devices.

We also simulated the transmission spectrum of an ADF using the FDTD solver (MODE Solutions). The experimentally measured and simulated transmission spectra at the drop and through ports of an ADF are shown in Fig. 5.7. For this simulation, we have used the actual parameters (ring length, coupling gap, material etc.) used in device fabrication. The estimated waveguide loss $(\approx 3 \mathrm{~dB} / \mathrm{cm})$ has also been included in the FDTD simulations. We observe that our FDTD simulations 

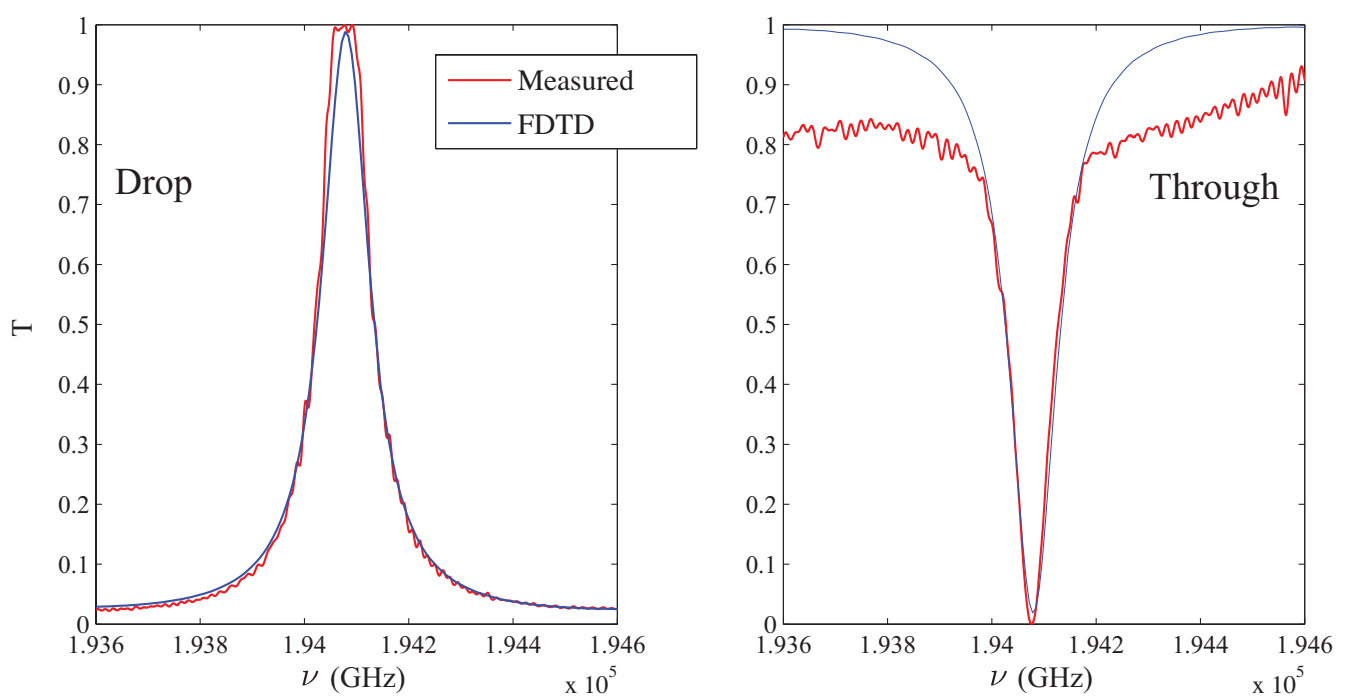

Figure 5.7: Comparison of a measured ADF spectrum with that simulated using FDTD. Actual fabrication parameters were used in the FDTD simulation. The two spectra show a very good match. The deviation seen in the through port spectrum is because of the wavelength response of fiber coupling.

match very well to the experimental measurements for all input light frequencies. We can therefore rely on our FDTD simulations for estimating the separation between ring waveguides to achieve a desired coupling rate $\kappa_{\text {ex }}$ or $J$.

\subsubsection{The 2D Lattice Design and Spectrum}

An SEM image of a $8 \times 8$ lattice sized device (Fig. 5.8(a)) shows the main site resonators, the link resonators and the input/output waveguides. For all our 2D lattice devices, we choose a magnetic field strength $\phi=2 \pi \alpha_{\mathrm{M}}=2 \pi \frac{1}{4}$. For $\alpha_{\mathrm{M}}=\frac{1}{4}$, there are three bandgaps occupied by edge states with winding numbers $+1,0$ and -1 respectively. The first and third bandgaps are, therefore, topologically non-trivial, separated by a large topologically trivial region. The edge states in these two topologically ordered bandgaps are the clockwise and the counterclockwise 

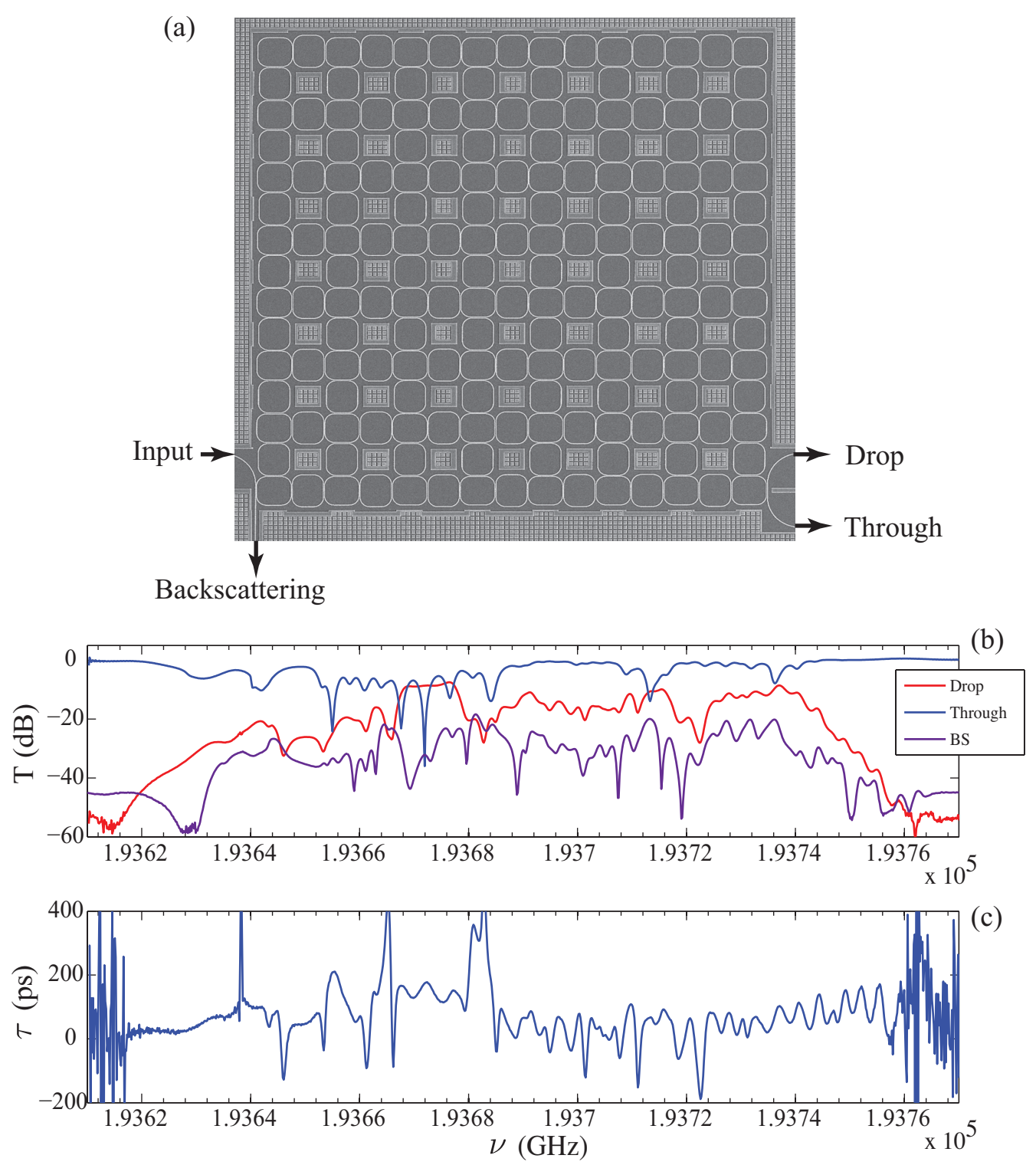

Figure 5.8: (a) SEM image of a $8 \times 8$ lattice. The input and output ports are also marked on the image. (b) Experimentally observed transmission spectrum at the drop, through and backscattering ports ports. (c) Delay spectrum at the drop port. 
propagating modes with opposite group velocities. This value of $\phi$ can be achieved using a shift of the link resonators in the $y$ direction, calculated as

$$
\xi=\frac{c}{\omega n_{\mathrm{eff}}} \phi
$$

For $\phi=\frac{2 \pi}{4}$, using $n_{\text {eff }}$ from the simulations, the required shift $\xi$ is $80 \mathrm{~nm}$. Similarly, the extra length $\eta$ of the link resonators required to make them antiresonant with the main rings is such that

$$
\eta=\frac{c}{\omega n_{\mathrm{eff}}} \pi
$$

which gives $\eta$ to be $160 \mathrm{~nm}$ at a wavelength of $1550 \mathrm{~nm}$. For these devices, we have used a coupling gap between the ring resonators as $180 \mathrm{~nm}$ and the coupling length between each pair is $7 \mu \mathrm{m}$. This results in $J \approx 32 \mathrm{GHz}$. The input and output waveguides are coupled to the lattice with a gap of $170 \mathrm{~nm}$ which yields $\kappa_{\mathrm{ex}} \approx 38$ GHz.

Experimentally observed transmission spectra at the drop, through and backscattering ports and the delay spectrum at the drop port for a $8 \times 8$ lattice is shown in Fig. 5.8(b,c). Although the long-edge region of the spectrum is expected to have a lower transmission than the short-edge because of the longer path it travels, we observe that the short-edge has a lower transmission. We will return to this issue in Section 5.4. However, we see that the long-edge region still has a higher delay due to traveling a longer path. The plot also shows the output at the back-scattering port which we see is $\sim 25 \mathrm{~dB}$ down from the drop port. 


\subsection{Imaging the Path of Light}

To demonstrate the presence of edge states, we use the surface scattering from ring resonator waveguides and hence, directly image the path followed by light in the lattice. A fraction of the light scattered by waveguide surface is directed to the microscope objective (positioned above the device for fiber coupling). The scattered light collected by the microscope objective is spatially imaged onto an GaAs-based IR CCD camera (320KTS-1.7RT from Goodrich, with $320 \times 256$ pixels). The output from the objective is split into two ports by a beam splitter. One of the ports is connected to the CCD camera in the visible domain (to assist in the fiber coupling) and the other to an IR CCD camera. We can, thus, simultaneously couple light to the device and image the device response to an excitation.

\subsubsection{CCD Camera Calibration}

We used an add-drop filter (ADF) to correlate the observed CCD Camera intensity to the ring resonator intensity. The ADF was fabricated on the same chip and with the same dimensions as the ring resonators in the $2 \mathrm{D}$ array devices. The transmission spectrum of the ADF was measured at the drop port using the LUNA OVA 5000, while simultaneously acquiring IR images at different wavelengths. The normalized transmission spectrum and the observed CCD camera intensity are shown in Fig. 5.9. The CCD camera intensity shown here is the intensity after integrating over the ring area. Since the transmitted power at the drop port is proportional to the ring resonator intensity, this plot is well correlated with the observed CCD cam- 


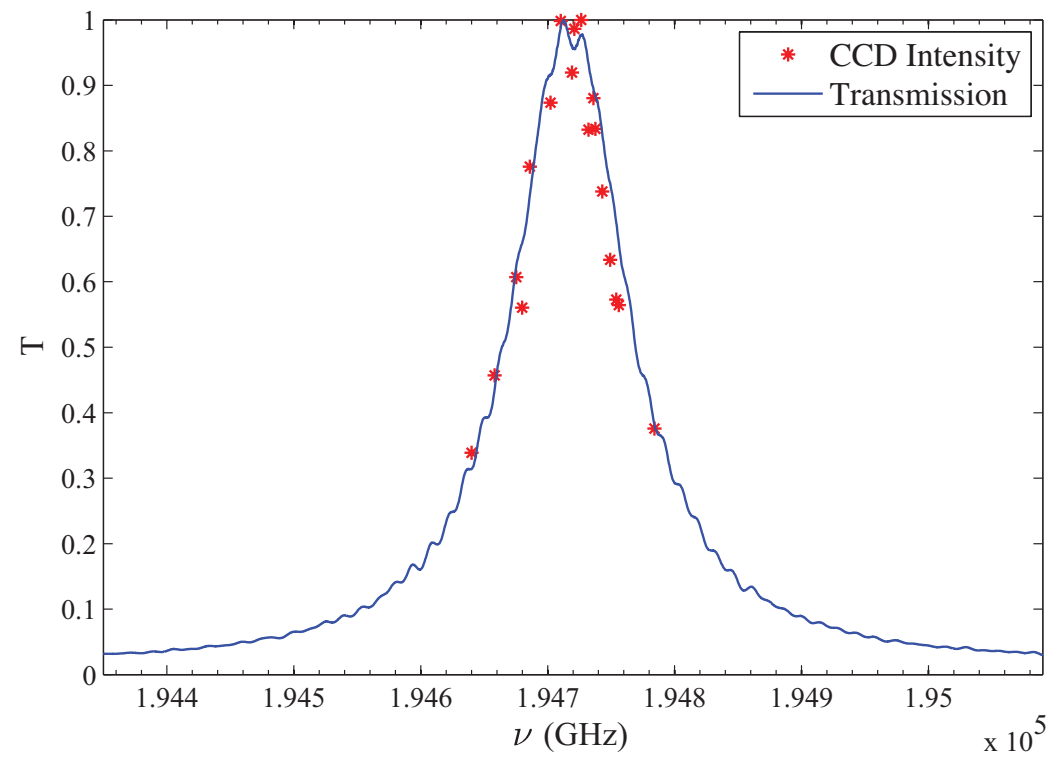

Figure 5.9: Intensity seen by the CCD camera as a function of input frequency, for an ADF. Since all the waveguide losses are proportional to internal energy, the camera intensity traces out the transmission spectrum.

era intensity. Therefore, even if a direct measurement of the light intensity traveling in a ring resonator sitting in a $2 \mathrm{D}$ array is not possible, the CCD camera imaging is a good proxy for the power distribution in ring resonators of the $2 \mathrm{D}$ array and hence, facilitates direct observation of edge states.

\subsubsection{Imaging Edge States}

To show the presence of edge states in our system, we used an $8 \times 8$ lattice sized device with a uniform synthetic magnetic field. Fig. 5.10 shows the measured transmission spectrum of the device. When the lattice is excited in specific regions of the spectrum (shaded red and green), the measured intensity distribution shows that the long and the short edges get excited. The transverse spatial width of the edge state was about one to two resonators, as observed both in experiment and 


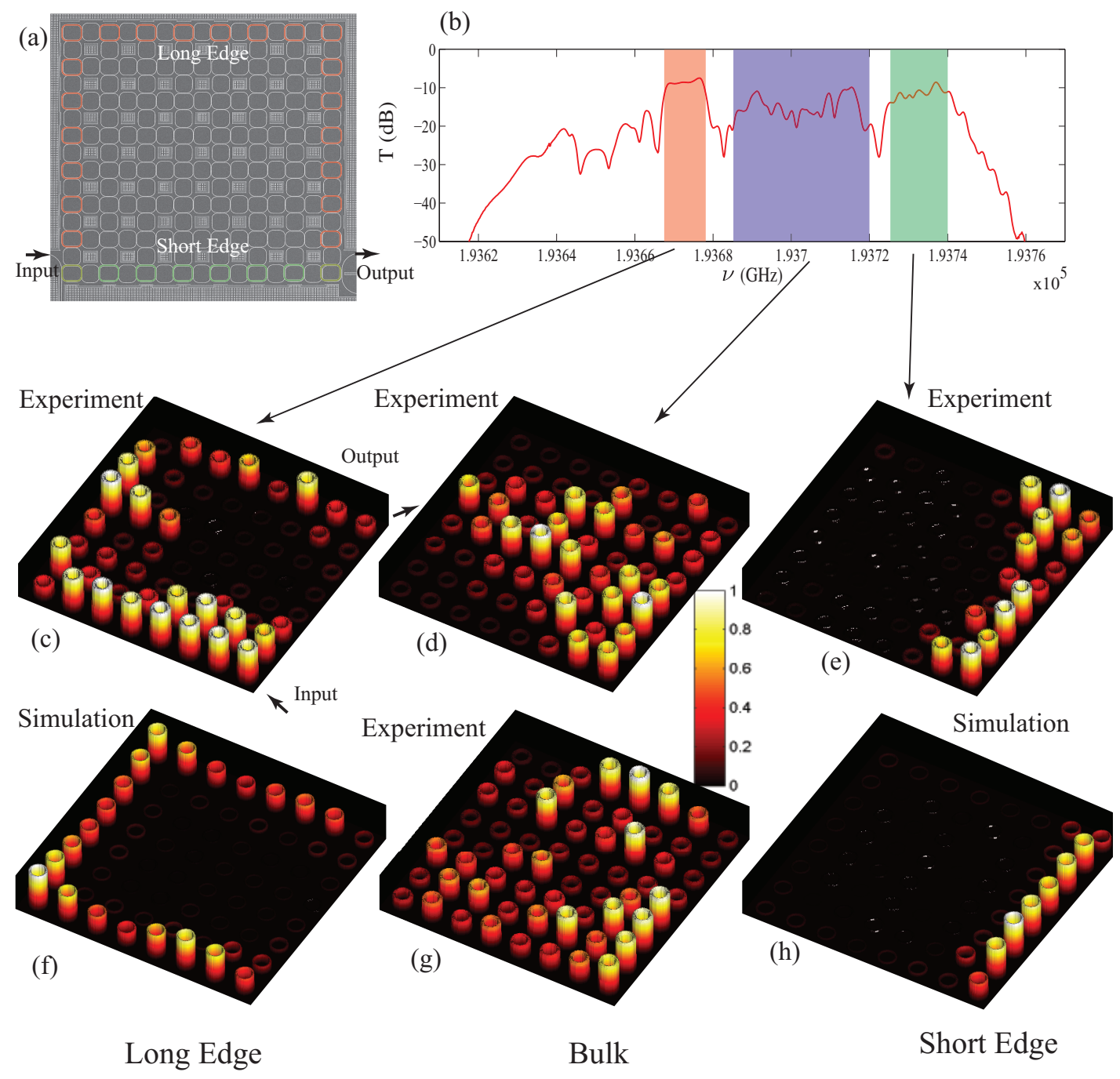

Figure 5.10: (a) SEM image of an $8 \times 8$ lattice. The path followed by short and long edge states is highlighted. (b) Transmission spectrum for this lattice. (c-h) Intensity plots for the experimentally observed and simulated short-edge, long-edge and bulk states. The experimental results match very well to the simulation results. The bulk state intensity plots shown here are the measured distributions at two very close lying input frequencies. For even a small change in the input frequency, bulk state pattern changes drastically [81]. 
simulation. The width is slightly greater in the experiment than in the numerical simulation due to the presence of intrinsic disorder in the fabrication which was not included in the simulation here (in Section 5.4, we will discuss simulations with disorder). For the simulation, we used parameters which were independently measured using single-ring devices. Because of topological protection, the light traverses two sharp corners in the long-edge band and leaves the system at the output port. In absence of topological protection, such sharp bends would cause enhanced scattering of light into the bulk of the lattice. Note that if the system were isolated (i.e., in the absence of input/output ports), the edge states could circulate around the entire system (as shown earlier in Chapter 2). When the system is pumped at a different frequency, bulk states are excited that do not have a particular shape (Fig. $5.10(\mathrm{~d}, \mathrm{~g}))$. More importantly, the edge-state profile does not significantly change over a frequency band broader than $5 \mathrm{GHz}$. In contrast, the profile of the bulk states changes dramatically when the frequency is changed even by $0.2 \mathrm{GHz}$. We attribute this remarkable difference to the topological protection of the edge states.

Note that the observed intensity in the CCD camera is a function of the light intensity traveling in the ring and the loss factor $\kappa_{\text {in }}$ of the ring. So if there are multiple resonators with equal light intensity traveling through them but with different $\kappa_{\mathrm{in}}$, the corresponding intensities read by the CCD camera imaging will be different. In our structures, since the standard deviation in $\kappa_{\text {in }}$ is of the order of $0.4 \kappa_{\text {in }}$, we expect some discrepancy between the simulated and the experimentally observed ring resonator intensities on the edge states. Such variation could be responsible for the discrepancy between the smooth edge state profile in the simulation and 


\section{(a)}

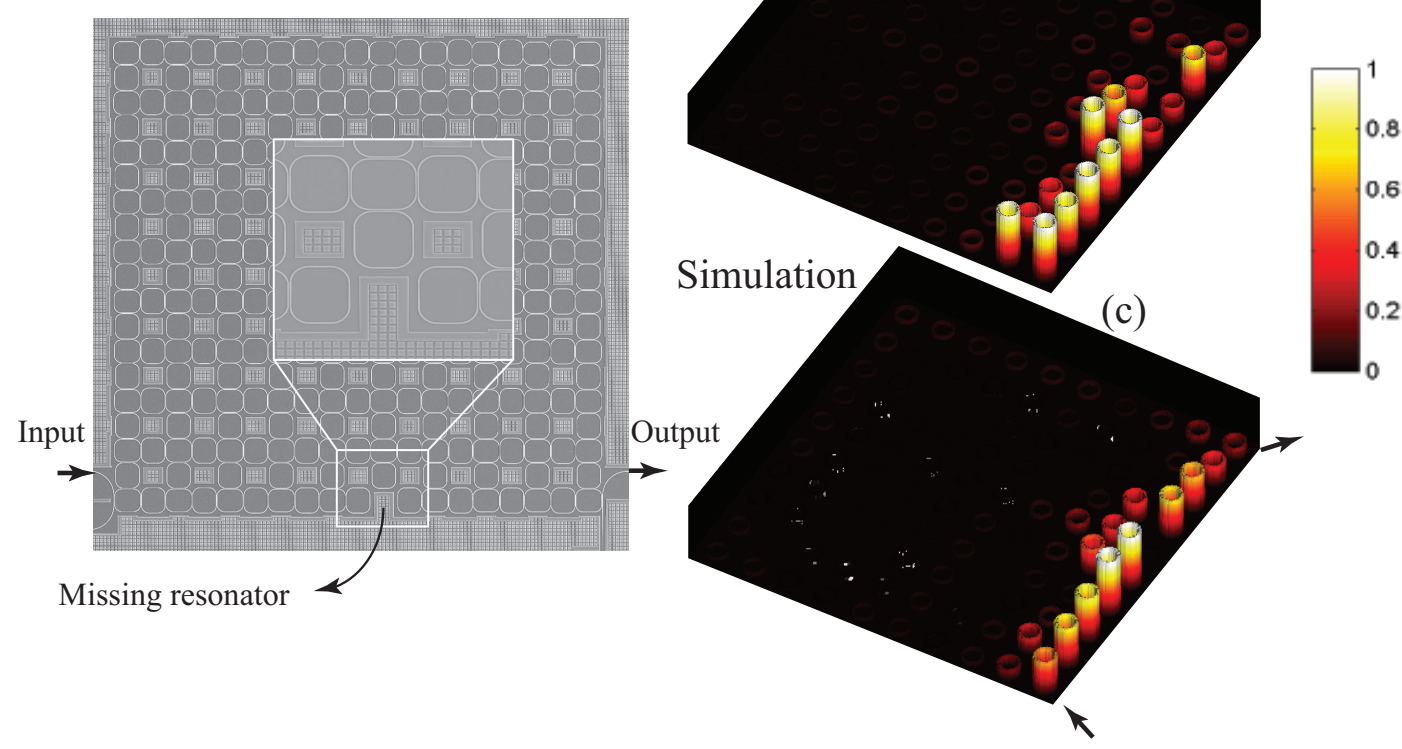

Figure 5.11: (a) SEM image of a $10 \times 10$ lattice with a missing resonator - a defect - along the short edge. (b,c) Observed and simulated intensity distribution showing the routing of edge state around the defect, without scattering into the bulk of the lattice [81].

inhomogenous profile of the edge states in the experimental images.

\subsubsection{Robustness of Edge States}

To demonstrate the robustness of edge states against an introduced disorder, we fabricated a $10 \times 10$ array with a missing resonator on one of the edges (Fig. $5.11(\mathrm{a}))$. As a result of topological robustness, it is expected that the edge state would bypass the impurity without scattering into bulk. When light was launched in the short edge band (over $15 \mathrm{GHz}$ ), we observed that the light entered at the bottom row from the left input corner, routed tightly around the disorder - in this extreme case of an entirely missing resonator - and travelled to the output port 
(a)

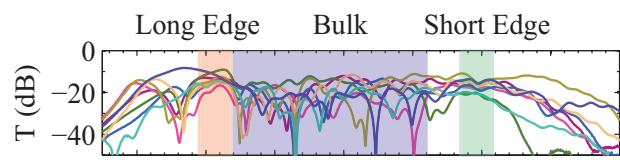

(b)

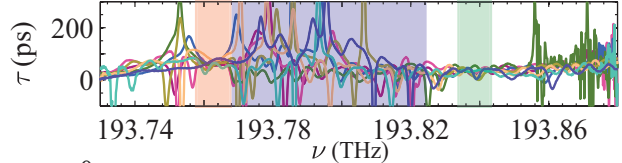

(c)

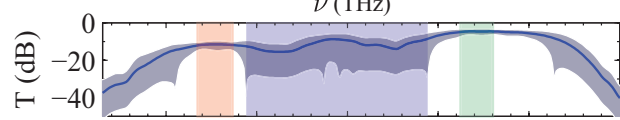

(d)

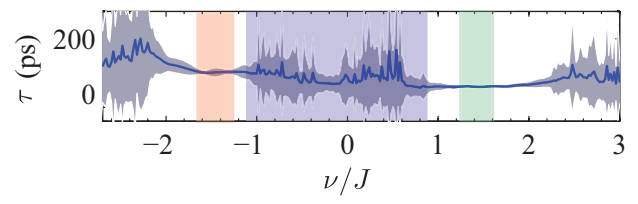

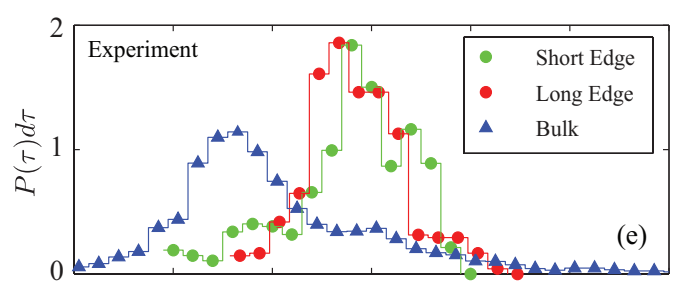

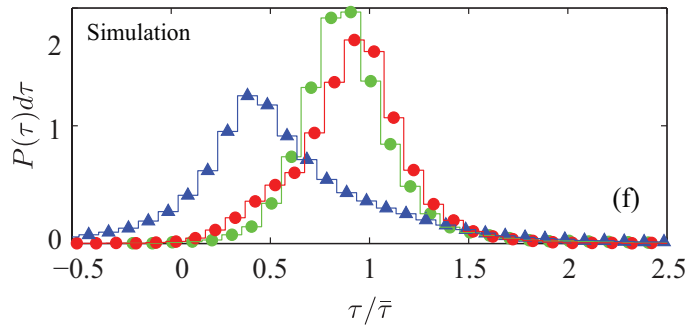

Figure 5.12: (a) Measured transmission and (b) delay-time spectra for eight $8 \times 8$ lattice size devices. The spectra have been normalized and shifted along x-axis to superpose them. Two regions with reduced variance in transmission and delay are indicated for the long edge (red) and the short edge (green). Noisy bulk states region is shown in blue. (c,d) Simulated transmission and delay with the average (solid blue line) and 95\% confidence band (grey shaded area) determined from the standard deviation across devices. (e,f) Measured and simulated delay distributions for edge and bulk states. Delay distribution for edge states is gaussian indicating diffusive transport. For bulk states the distribution is asymmetric, showing localized transport. Data is averaged for 8 devices. The delays are normalized to the average (rms) and the overall delay distribution is normalized to in-band average and the delay distribution is normalized such that the area under the curve is unity [82].

without entering into the bulk (Fig. 5.11(b)), in good agreement with simulation (Fig. 5.11(c)). The residual presence of light at the missing resonator location in

Fig. 5.11(b) is due to background noise.

\subsection{Robust Transport of Photons}

Having shown the presence of edge states in our system using a qualitative imaging technique, we now quantitatively demonstrate the robust transport properties of edge states using statistics of transmission and delay measurements made on a number of devices. Fig. 5.12(a,b) shows the observed transmission and de- 
lay spectra at the drop port for eight different $8 \times 8$ lattice size devices. While the spectra differ significantly because of intrinsic fabrication variations in waveguide dimensions, we can already see the first manifestation of robust edge states in the form of two regions with suppressed variance across devices, in both the transmission and delay spectra. Since edges states are topologically constrained to travel along the lattice edge, device-to-device fabrication variations in system parameters do not affect the edge state wavefunctions as much as they do for bulk states. Edge states, therefore, show reduced variation. Using numerical modeling including our measured values for disorder, as shown in Fig. 5.12(c,d), we can identify these regions as due to the long-edge and the short-edge state. Because of the intrinsic spread in resonance frequencies resulting from fabrication disorders, the measured and simulated spectra have been shifted along the frequency axis to superpose them. Each transmission and delay spectrum shown here is normalized to its corresponding measurement made away from the resonance band, at the through port. The measurements thus normalized give the actual transmission and delay incurred only through the lattice and excludes those in the coupling waveguides and connecting fibers.

\subsubsection{Delay Distribution}

Next, we analyze the delay distribution to distinguish edge state transport from bulk states. This approach provides an unequivocal signature of localization $[25,83$, 84]. Fig. 5.12(e,f) show the measured and simulated delay distributions for the edge 
and bulk states in $8 \times 8$ lattice sized devices and highlight the remarkable difference between edge and bulk states. For edge states, the delay distribution normalized to its average (in actuality, we used root mean square to allow for negative delay values), is essentially gaussian with width independent of system size. This behavior is characteristic of diffusive transport as seen previously in one-dimensional systems $[24,85]$. The bulk state distribution is, however, asymmetric with the most probable value being less than the average. This feature is reminiscent of transport governed by localization which has also been observed earlier in the microwave regime for onedimensional systems $[25,84]$. For localized transport, the delay spectrum exhibits spikes (see 5.12(b)) which manifest in the asymmetric delay statistics. These spikes appear due to resonant tunneling through (delocalized) necklace states which are common to finite-size open systems [86]. Therefore, even in the presence of loss, the delay distribution can clearly differentiate two different regimes of transport in the same photonic system. Our measured results show a good match with numerical modeling. We observe similar behavior for other lattice sizes as well, as shown in Fig. 5.13 for $15 \times 15$ sized devices.

\subsubsection{Comparison with 1D Array}

A test to further establish the topologically-protected nature of edge states would be a comparison of the transmission scaling with system size for an edge state against that of a topologically trivial $1 \mathrm{D}$ system $[54,55]$, both with similar degrees of disorder. Fig. 5.14 shows the observed transmission and delay spectra 

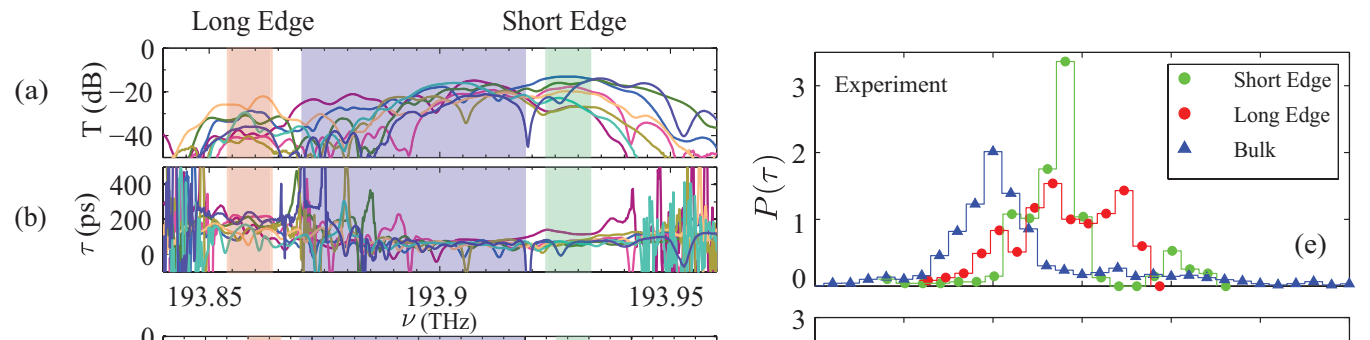

(c)
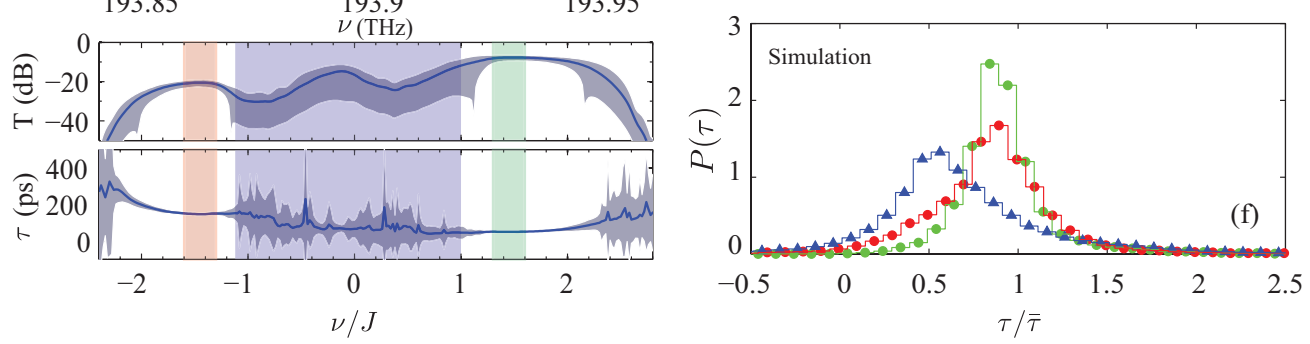

Figure 5.13: (a,b) Measured, and (c,d) simulated transmission and delay-time spectra for eight $15 \times 15$ lattice size devices. (e,f) Measured and simulated delay distribution for edge and bulk states. We see similar behavior as with $8 \times 8$ lattice sized devices [82].

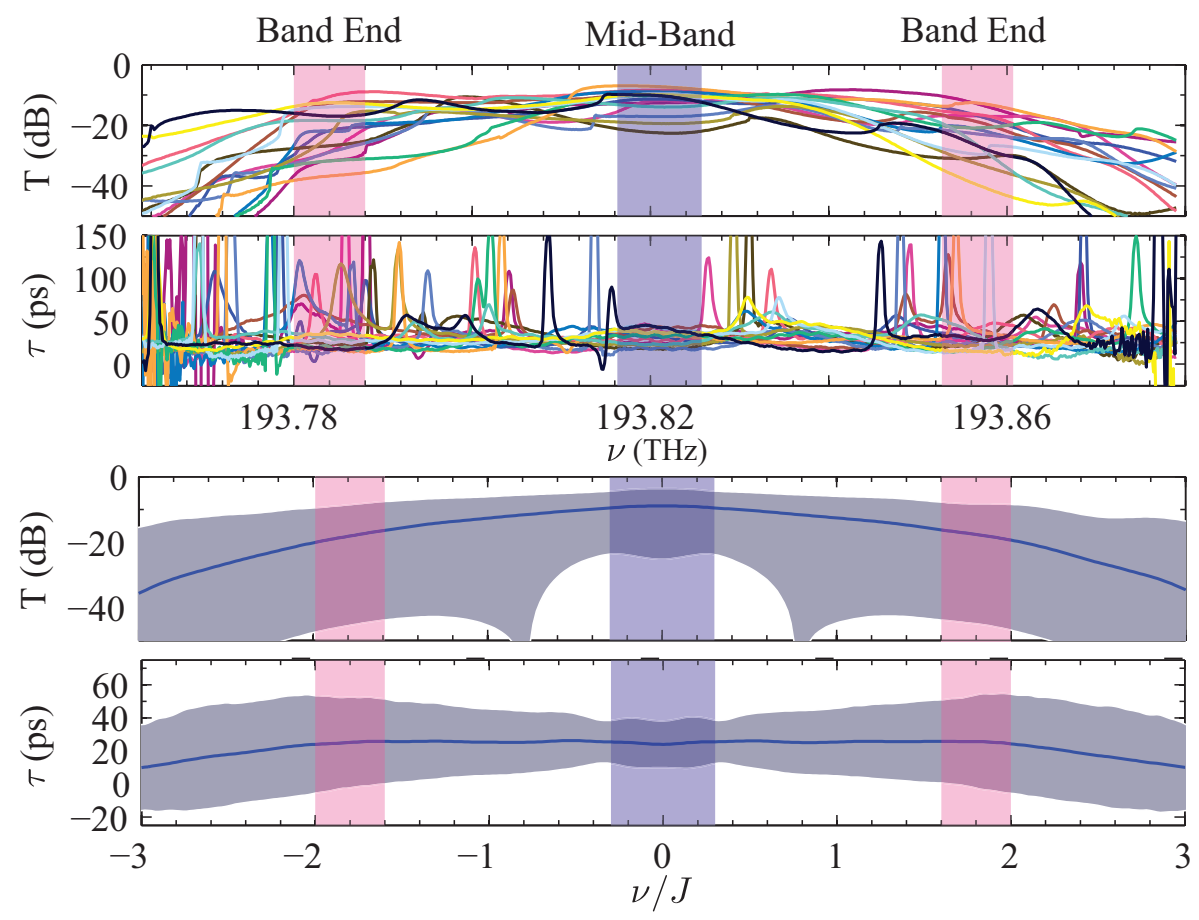

Figure 5.14: (a,b) Measured transmission and delay spectra for ten, 10 ring 1Darray devices. (c,d) Simulated spectra. There are no regions which are robust against disorder. 
for ten 10-ring devices. Fabrication-induced disorder in a $1 \mathrm{D}$ ring resonator array leads to a spread in the resonance wavelengths of the resonators. This impedes the forward propagation of light, increases back-reflection, i.e., less light is coupled into the array and, hence, the transmission at output is reduced [56]. Ultimately, as the array length increases, Anderson localization halts transmission of the light (Fig. 4.9) [59, 87-89]. Edge states, on the other hand, are unidirectional and immune to reflection caused by disorder. Therefore, transmission through edge states is expected to be less affected.

Fig. 5.15(a) shows the measured average transmission and its standard deviation across a number of chips (95 in total) for the long edge state band in 2D lattice and the mid-band of the $1 \mathrm{D}$ array as a function of system size, i.e. the number of resonators travelled from input to output (excluding the link resonators). Transmission in both the long-edge state as well as the 1D system decays exponentially with system size. A linear fit to measured transmission (in $\mathrm{dB}$ ) in the long edge band gives the decay slope as $-0.75(20) \mathrm{dB}$ per ring but for $1 \mathrm{D}$ transmission, the slope is $-0.93(16) \mathrm{dB}$ per ring, where uncertainties represent one sigma standard deviations. Transmission along long edge state can be seen to decay at a slower rate as compared to $1 \mathrm{D}$ transport. Simulation results using the experimentally estimated parameters are also presented in the figure. The simulated transmission decay slopes are $-0.66(2)$ and $-1.06(5) \mathrm{dB}$ per ring for long edge state and 1D, respectively. The experimental and simulation results are seen to agree, given that the number of devices measured for each lattice size is only $\approx 8$ versus the 5000 realizations for each simulation. To differentiate the decay of transmission with system size result- 

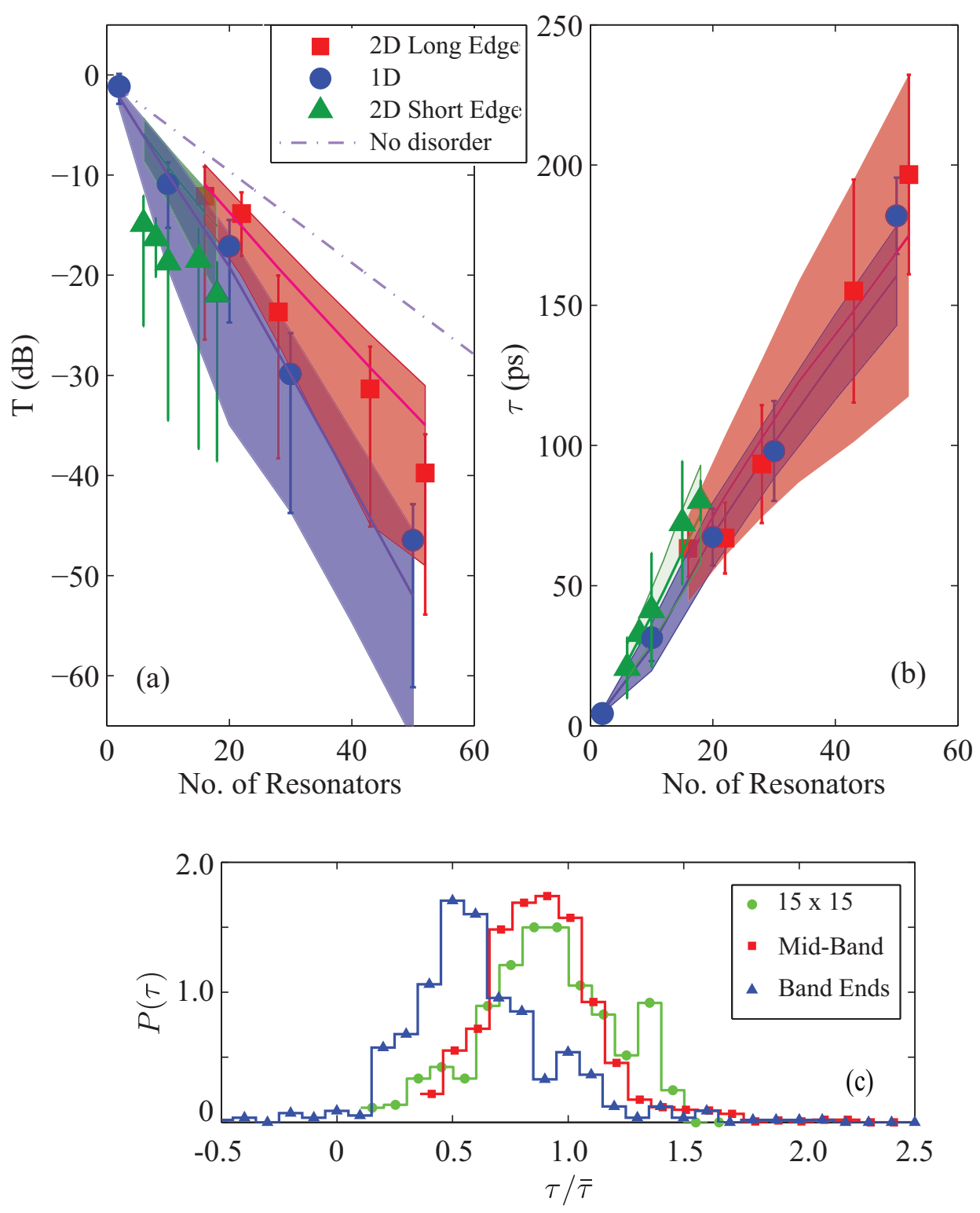

Figure 5.15: (a) Transmission and (b) delay-time scaling for 2D and 1D devices. Solid markers with error bars are the measured average and standard deviation $(65 \%$ confidence band) values. Solid lines with shaded areas are the simulated average and standard deviation. Also shown is the transmission when there is no disorder in the system. For 2D data, we measured $(7,8,9,8,8)$ number of chips for $(6 \times 6,8 \times 8$, $10 \times 10,15 \times 15,18 \times 18 \mathrm{ring})$ sized devices respectively. For the $1 \mathrm{D}$ data, we measured $(11,15,11,12,6)$ number of chips for $(2,10,20,30,50)$ ring devices. (c) Delay statistics for long edge of $15 \times 15$ lattice sized devices, mid-band and band-ends of 30-ring $1 \mathrm{D}$ devices. The long edge and mid-band of 1D devices show diffusive transport. The band-ends in 1D devices however show localization [82]. 
ing from resonator losses characterized by $\kappa_{\text {in }}$, from losses due to disorder - both resulting in exponential attenuation - we plot the simulated result for transmission without disorder (presented as a dashed line). In that zero disorder limit, both the $2 \mathrm{D}$ and $1 \mathrm{D}$ systems are similarly attenuated by loss with a decay rate of $0.46 \mathrm{~dB}$ per ring. We therefore observe that disorder affects both 2D and $1 \mathrm{D}$ systems, but transport in edge states is less susceptible to disorder.

Fig. 5.15(b) shows the measured and simulated average delay and its standard deviation for the short-edge and long-edge-state-bands. The measured delay, when plotted against the number of rings on the short and long edges of the lattice, increases linearly with a slope 3.9(9)ps and 5.4(1.0)ps per ring for long and short edge states, respectively. The simulated delay slopes are 3.2(2)ps and 4.4(1)ps per ring, respectively. Again, the experimental results are in agreement with the simulation. Also shown in the figure, for comparison, is the measured delay in $1 \mathrm{D}$ devices. That delay follows the same scaling as the edge states. However, it can be seen that the standard deviation in delay for 1D devices is less than that for edge states. This is contrary to the case of a uniform magnetic field where the standard deviation of delay in edge states remains smaller than in a 1D system [15]. Using simulations we have verified that this is due to the fabrication disorder of $\Delta \phi$ in the 2D lattice. We further compare the delay distribution for edge states and a $1 \mathrm{D}$ system. The normalized delay distributions for long-edge states in a $15 \times 15$ lattice and for midband and band-ends of a 30 ring 1D array are shown in Fig. 5.15(c). We see that for both, edge states and the mid-band of a 1D array, the transport is diffusive, the distribution is gaussian and the width of the distribution is independent of system 


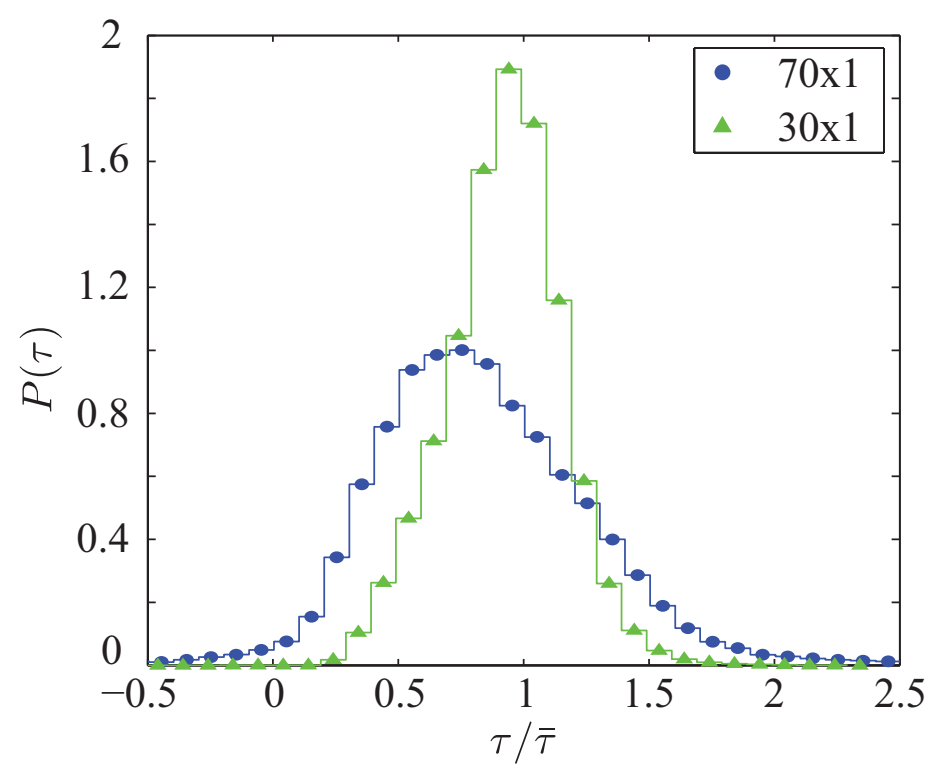

Figure 5.16: Simulation results showing localization in a 1D array of 70 rings, in the presence of disorder.

size. However, the band ends of the 1D array are localized. Using simulations, we also find that as the array length increases beyond 70 rings, even the mid-band of the 1D system shows localization (Fig. 5.16).

From simulation, we infer that the main disorder terms affecting transmission in a $2 \mathrm{D}$ lattice are $\Delta \omega_{0}$ and variations in the otherwise uniform magnetic field, i.e. $\Delta \phi$, whereas for a $1 \mathrm{D}$ array only the first term is applicable since there is no magnetic field. In the absence of $\Delta \phi$, the transmission in the edge state would be even closer to the dashed line with no disorder.

The short-edge transmission in our system was, however, consistently found to be much lower $(\approx 8 \mathrm{~dB}$ for $6 \mathrm{x} 6$ devices $)$ than expected using simulations, but it tends to match the simulation results for bigger sized devices. We expect that this is the result of some systematic problem with our fabrication process which couples 


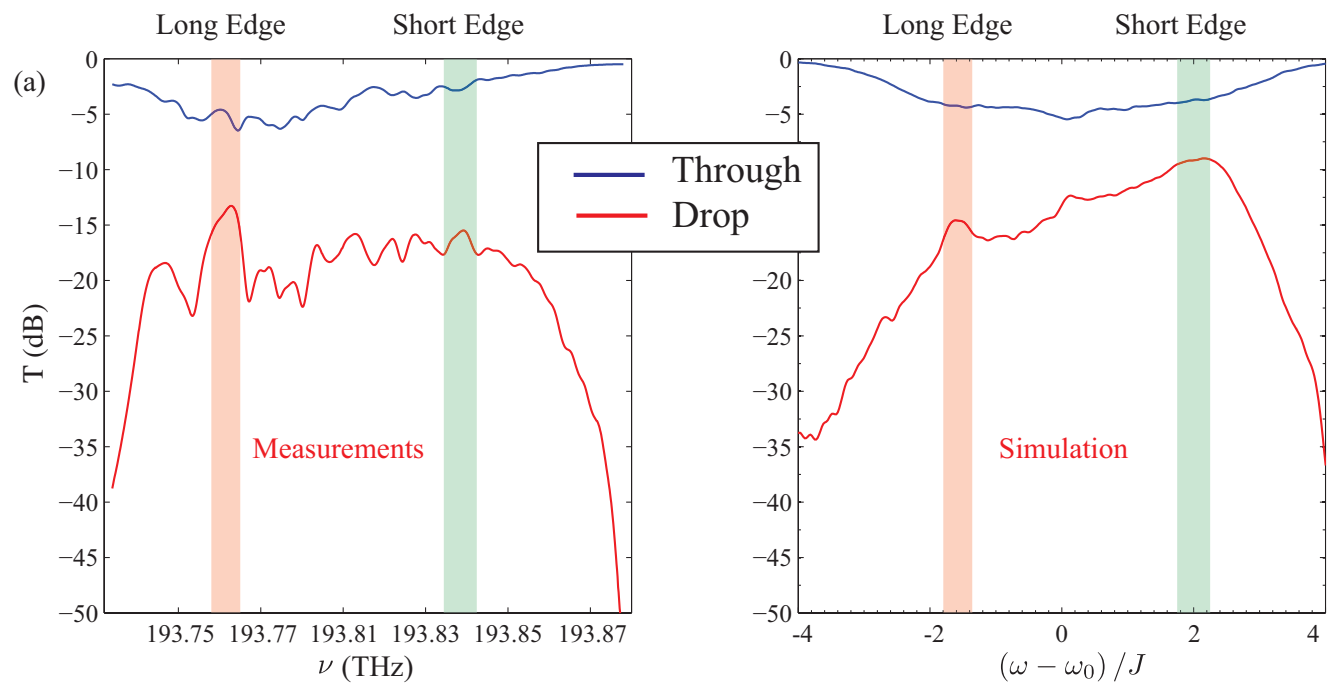

(b)

Figure 5.17: Mean transmission at the drop and through ports for $8 \times 8$ lattice sized devices, (a) Measured, (b) Simulated. The experimental results clearly show that the power coupled to the lattice around short edge region is less than that at the long edge region.

less light to the short edge at the input port and hence, produces a non-zero intercept on the transmission axis. Using through port data, we verified the reduction in coupling efficiency for the short edge band, for all devices (Fig. 5.17).

\subsubsection{Disorder Characterization and Numerical Simulations}

To characterize the system parameters, we use the simpler one ring devices, i.e., the $\mathrm{ADF}$ and the $\mathrm{APF}$, designed with the same parameters as those of the rings in the lattice. Transmission measurements were made on 26 single ring ADFs on different chips. Using these measurements $\kappa_{\text {in }}, \kappa_{\text {ex }}$ and $J$ were measured to be $2.35 \mathrm{GHz}, 37.8 \mathrm{GHz}$ and $32.0 \mathrm{GHz}$ with relative standard deviations of $20 \%, 4 \%$ and $4 \%$, respectively. The magnetic flux $\phi$ is designed to be $\frac{\pi}{2}$. For $1 \mathrm{D}$ devices, the measured bandwidth was less than that expected from simulations with $J=32$ 
GHz. Therefore, for $1 \mathrm{D}$ simulations, a corrected value of $J=25 \mathrm{GHz}$ was used. A similar procedure was used earlier to estimate $J$ for a 1D array of coupled resonators in [55]. Fabrication errors also result in a variation of the resonance frequency $\nu_{0}$ of the rings in a given lattice. The standard deviation $\Delta \nu_{0}$ was estimated using transmission measurements on three chips with five all-pass filters each, with a physical separation of the APFs commensurate to rings in a lattice. $\Delta \nu_{0}$ can then be used to calculate the deviation of optical path length and hence, $\Delta \phi$ in the link rings. $\Delta \nu_{0}$ was estimated to be $27.5 \mathrm{GHz}$ and $\Delta \phi$ to be 0.1 .

For numerical simulations, we use the single-mode approximation as discussed earlier. To include lattice disorder into this Hamiltonian, we impose random variations on each of the parameters with a gaussian probability distribution around the mean. For each numerical realization of the lattice, each resonator ring has noise added to its resonance frequency $\omega_{0}$, coupling rate $J$ to neighboring resonators and also to the magnetic phase acquired in hopping along the $x$-axis. To get mean transmission and delay, we then average the results over 5000 realizations for each device type. Required simulation parameters and their deviations, characterizing the system and its disorder, have been measured using multiple add-drop filters as described above.

\subsubsection{Calibration of Spectra and Device Yield}

Each transmission and delay spectrum shown in the previous section is normalized to the corresponding measurement made away from the resonance band, 
at the through port. The measurements thus normalized give the actual transmission and delay incurred only through the lattice and excludes those in the coupling waveguides and connecting fibers. Because of the intrinsic spread in resonance frequencies resulting from fabrication disorders, the measured and simulated spectra have been shifted along the frequency axis to superpose them. Since the spectra are expected to be disparate in the bulk region [15], we can rely only on the edge state regions to superpose them. For measured spectra, we therefore first do a manual coarse shift to align similar looking features in the expected edge state regions of the spectra. This accounts for $\Delta \nu_{0}$ across various chips (which is much greater than $\Delta \nu_{0}$ for a given lattice). Subsequently we analyze the standard deviation of transmission and delay across devices as a function of frequency and find that the edge states are evident as regions with reduced noise. To verify this evidence for edge states and to align them further, we require an algorithm based on quantitative measurements of transmission $(\mathrm{T})$ and delay-time $(\tau)$. Weighted delay time $W(\nu)=T(\nu) \tau(\nu)$ is one parameter that accounts for both our measurements and has been used extensively to study transport properties in random media $[25,85]$. For completely random transport, as is the case for bulk states, we expect increased variations in $T(\nu)$ and $\tau(\nu)$ and hence, also in $W(\nu)$ as a function of frequency. On the contrary, transport through the edge states band follows a definite path and should, therefore, display regions with reduced variance in $W(\nu)$. We accordingly use the standard deviation of $W(\nu)$ to look for edge state regions and align the measured spectra. 
Each spectrum is shifted such that $f_{\nu}$ given by

$$
f_{i}=\frac{\sigma_{\nu}\left(T_{i}(\nu)\right)}{\overline{T_{i}(\nu)}}+\frac{\sigma_{\nu}\left(\tau_{i}(\nu)\right)}{\overline{\tau_{i}(\nu)}}
$$

where $i$ refers to device index, is minimized in the designated edge state bands. The bandwidth of the long edge is found to be $\approx 10 \mathrm{GHz}$, independent of the device size, while the short edge is wider $(12.5 \mathrm{GHz}-19 \mathrm{GHz})$. For our analysis, we fix the bandwidth of the short- and long-edge regions to be $10 \mathrm{GHz}$ for all devices. For simulated spectra, we follow exactly the same protocol except for the course shift which is not required. The spectra have not been shifted along y axis and there is no re-scaling of the spectrum.

Table 5.1 shows the detailed device yield. For 2D devices, the device yield was found to be $\approx 23 \%$. For $1 \mathrm{D}$ devices (other than 50 rings), the device yield was $100 \%$. For 50 ring devices, the yield was $\approx 50 \%$. The devices with a very noisy or attenuated spectrum were considered as bad.

\subsection{Summary}

In this Chapter, we reported the experimental realization of our system of a 2D lattice of coupled ring resonators. We started with a discussion of the fabrication procedure followed by the experimental setup which measure the transmission and delay spectra of the devices. The setup used an IR CCD camera which allowed us to directly show the presence of edge states in our system. We demonstrated the robustness of edge states by deliberately introducing a defect into the lattice. The edge state routes around the defect and do not scatter into the bulk. Finally, using 


\begin{tabular}{|c|c|c|c|c|}
\hline Device size & Worked & Bad & Did not & Total \\
\hline $\mathbf{6} \times \mathbf{6}$ & 7 & 28 & 0 & 35 \\
$\mathbf{8} \times \mathbf{8}$ & 8 & 27 & 0 & 35 \\
$\mathbf{1 0} \times \mathbf{1 0}$ & 9 & 26 & 0 & 35 \\
$\mathbf{1 5} \times \mathbf{1 5}$ & 8 & 27 & 0 & 35 \\
$\mathbf{1 8} \times \mathbf{1 8}$ & 8 & 27 & 0 & 35 \\
$\mathbf{2} \times \mathbf{1}$ & 11 & 0 & 21 & 35 \\
$\mathbf{1 0} \times \mathbf{1}$ & 15 & 0 & 20 & 35 \\
$\mathbf{2 0 \times \mathbf { 1 }}$ & 11 & 0 & 24 & 35 \\
$\mathbf{3 0 \times 1}$ & 12 & 0 & 23 & 35 \\
$\mathbf{5 0 \times 1}$ & 6 & 7 & 22 & 35 \\
\hline
\end{tabular}

Table 5.1: Number of devices measured, good and bad, for each device type. 
delay distributions and comparison with topologically trivial states, we quantitatively established the robustness of edge states against fabrication induced disorder. We showed that the edge states are less susceptible to localization. 


\section{Chapter 6: Measuring Winding Number in Photonic Systems}

Edge states are characterized by a topologically invariant integer, the winding number. As discussed in Chapter 2, the winding number $t_{n}$ for the edge-states is calculated using Diophantine equations as $[8,16,29,39]$

$$
n=q s_{n}+p t_{n} ; \quad\left|t_{n}\right| \leq \frac{q}{2} .
$$

Here, the uniform magnetic flux threading the lattice is $\phi=2 \pi \alpha_{\mathrm{M}}$, where $\alpha_{\mathrm{M}}=p / q$. The energy spectrum for this lattice, the Hofstadter butterfly, shows that there are $q$ energy bands separated by $q-1$ band-gaps. For a finite lattice, the bandgaps are occupied by edge states. $n$ is the gap index $(1 \leq n \leq q-1)$, with the lowest energy bandgap labelled $n=1 . s_{n}$ is an integer which satisfies the above equation. A nonzero winding number indicates topologically non-trivial states. For photonic edge states, the integer quantization of winding number is analogous to the quantization of Hall conductance in electronic systems. In this Chapter, using a square annulus geometry, we show that the winding number of the edge states can be determined using Laughlin's gauge invariance approach (refer Section 2.3) and the transmission spectrum measurements. We also compare the square annulus to a simple ring geometry where the eigenstates possess a non-zero winding number but are not robust. 


\subsection{Winding Number of Edge States in a Square Annulus}

For electronic systems, the winding number of the edge states is related to the quantized Hall conductance through the bulk-edge correspondence principle $[8,16]$. Therefore, a direct measurement of the Hall conductance yields the winding number of the edge states. Since the concept of conductance does not apply for photonicbosonic-systems, measuring the winding number of photonic edge states using regular conductance methods is not possible. However, we can still use Laughlin's adiabatic pump in a circular annulus (the Corbino geometry - Fig. 2.5) to measure the winding number.

In the presence of a uniform magnetic field threading the annulus, the spatial wavefunction of the electron cyclotron orbits is labelled by the energy level $n$ (the Landau level) and the $z$-component of the angular momentum, $m$ (see Section 2.3). The electron cyclotron orbits are localized to the perimeter of a circle of radius $\sqrt{2 m} l_{0}$, where $l_{0}$ is the magnetic length $[7,31,37]$. Introduction of an additional magnetic flux $\Phi$ in the central hole causes a gauge transformation of the vector potential. Each unit flux quanta increase in the magnetic flux raises the angular momentum value by one. The electron wavefunction for a given $m$ thus expands outwards and takes the position once occupied by its neighbor corresponding to angular momentum $(m+1)$. In this process, $n$ electron states are adiabatically pumped from the inner edge to the outer edge, where $n$ is the number of filled Landau levels.

The spatial transport of edge states can also be observed in the energy spec- 
trum of the system [29], where, for a unit quanta change in flux, edge states shift by their winding number. A measurement of the energy spectrum as a function of external flux, thus, reveals the winding number of edge states. A similar scheme to measure the winding number of edge states has been demonstrated in the microwave domain using a 2D directed network [90]. Adiabatic pumping in photonic systems has also been achieved in 1D arrays of coupled waveguides [91]. A direct measurement of the winding number of edge states in the optical domain has still remained elusive. Here, following a theoretical proposal to measure the winding number in our system [29], we report the first experimental measurement of the winding number for photonic edge states.

Mimicking the Corbino geometry used in [7], we design a square annulus of ring resonators, coupled by link rings (Fig. 6.1). The link rings have been shifted vertically to achieve a uniform synthetic magnetic field with flux $\phi=2 \pi / 4$, in exactly the same fashion as we did for the square lattice in Fig. 4.4. To introduce an external magnetic flux into this system, we use the fact that the external flux essentially results in an Aharanov-Bohm phase in the annulus. This additional phase can be incorporated into the lattice by placing metal heaters above the link ring waveguides. In the simplest form, as the name suggests, heaters are high resistance metal pads (Fig. 6.2). A current flowing through the pad generates heat which in turn modulates the effective refractive index of silicon waveguide and hence, the phase incurred by light travelling through it. The heaters are placed such that any closed path around the central hole results in equal Aharanov-Bohm phase, given all the heaters are the same and carry equal current. This geometry is then equivalent 


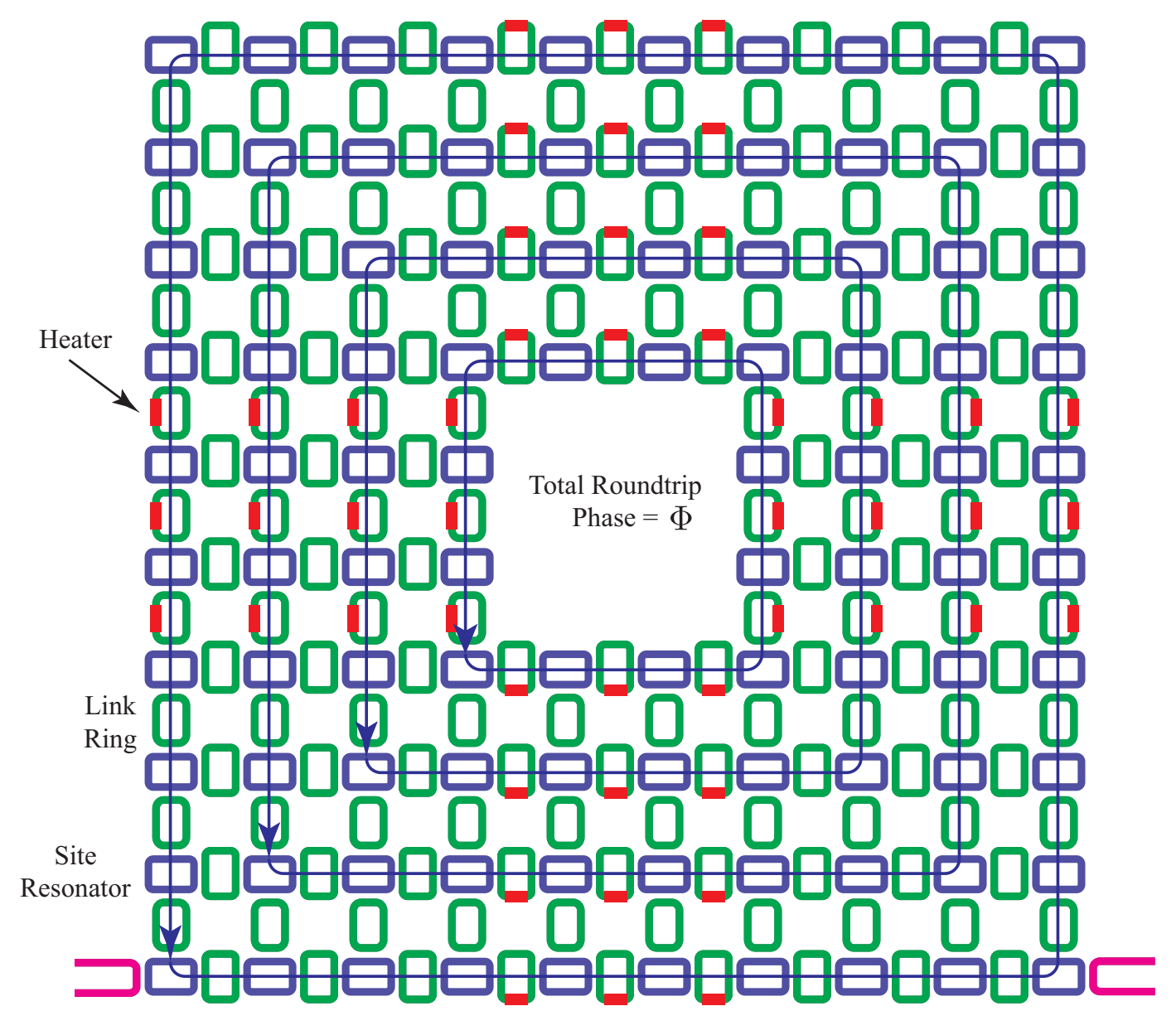

Figure 6.1: Square annulus geometry to measure the winding number of edge states. The total phase $\Phi$ introduced by heaters (placed on link-resonator arms) is the sum of individual phases incurred in link resonator arms, in any closed path. Heaters (shown in red) are placed such that the accumulated phase $\Phi$ is same for any path enclosing the center hole. 


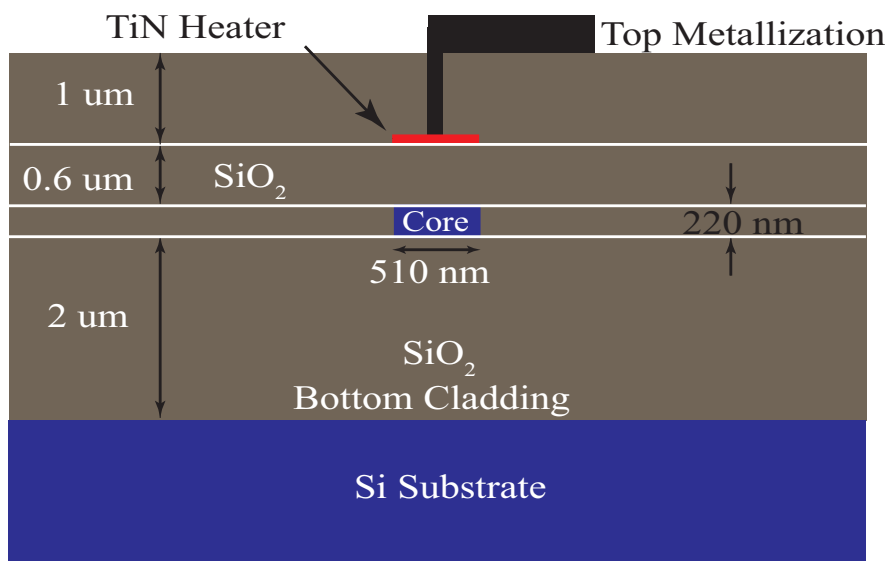

Figure 6.2: Waveguide cross-section with heaters. Heaters are $110 \mathrm{~nm}$ thick TiN pads. The heater pads are connected to outside metallization to provide electrical connectivity.

to the Corbino geometry (Fig. 2.5) with a uniform magnetic field in the annulus and variable magnetic flux in the central hole.

For this system, since $\alpha_{M}=1 / 4$, there are 4 bulk bands separated by 3 bandgaps. Using Eq. 6.1, the edge states in the first bandgap have $t_{1}=+1$, whereas for those in the third band $t_{3}=-1$. The states in the second bandgap have a winding number zero and hence are topologically trivial. Moreover, the system now supports two sets of edge-states in each bandgap, on the outer edge and the inner edge, which circulate around the lattice in opposite directions.

Fig. 6.3 shows the eigenvalues of this system as a function of the external phase $\Phi$, the total phase introduced by a loop of heaters. We observe that with an increase in the external flux, all the edge states shift outwards or inwards. The CW-propagating outer edge states in the first bandgap move toward lower energies, whereas the CCW-propagating outer edge states in the third bandgap move toward higher energies. The inner edge states move exactly opposite to the outer edge 


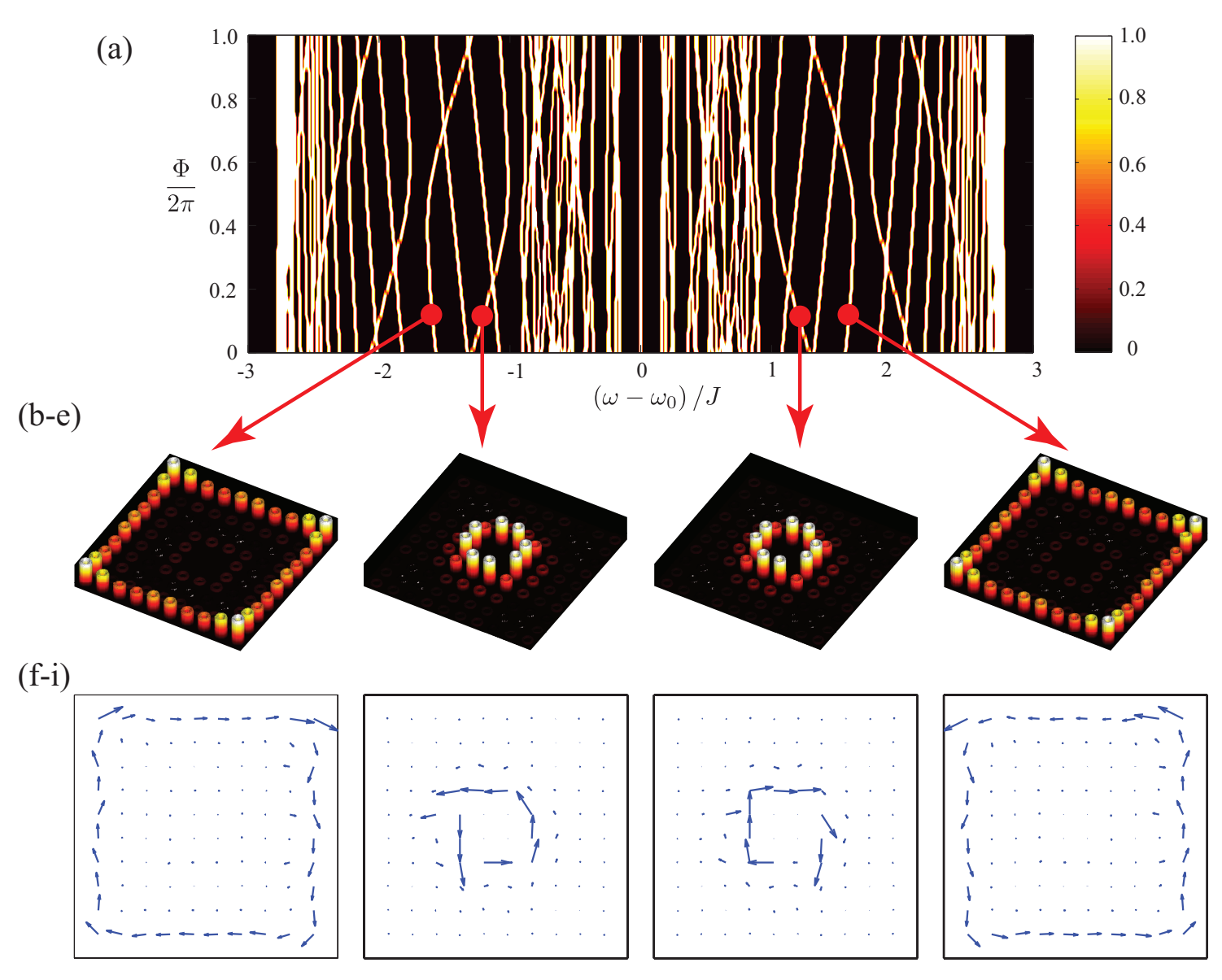

Figure 6.3: (a) Shifting of eigenvalues as a function of external magnetic flux $\Phi$. The annulus now supports two sets of edge states, at the outer (b,e) and the inner edges $(\mathrm{c}, \mathrm{d})$. In a given bandgap, the outer $(\mathrm{f}, \mathrm{i})$ and the inner $(\mathrm{g}, \mathrm{h})$ edge states circulate around the lattice in opposite directions because of their opposite group velocity. Furthermore, in the energy spectrum (a), the clockwise and the counterclockwise circulating states move in opposite directions because of their winding number which is \pm 1 . 
Measured
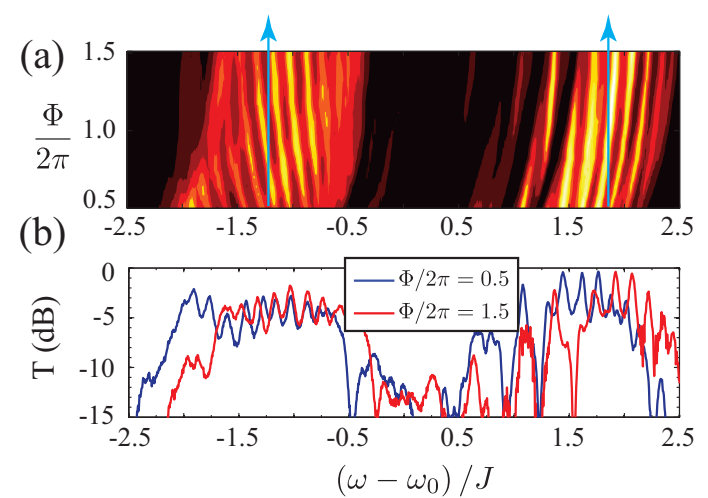

Simulated

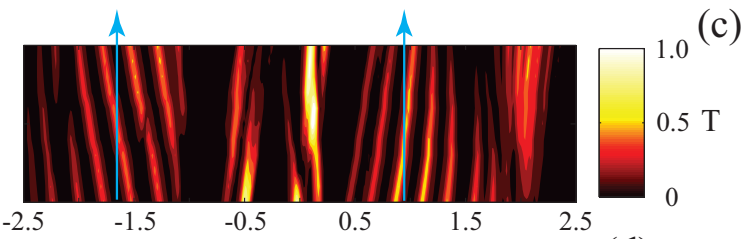

(d)

Figure 6.4: (a) Measured and (c) simulated transmission (in linear scale) as a function of phase $\Phi$ and frequency $\omega$. As the phase $\Phi$ increases, the edge state peaks in the transmission move away from the center. For a $2 \pi$ increase in magnetic flux, the edge states move by one. (b) Measured and (d) simulated transmission spectra (in $\log$ scale) for $\Phi / 2 \pi=0.5,1.5$. For this $2 \pi$ increase in flux, the measured spectra match approximately in the edge state regions. The transmission spectra have been normalized such that the maximum transmission is unity.

states. More importantly, for a $2 \pi$ increase in magnetic flux, each set of states shift exactly by one, their winding number. The direction of shift is indicated by the sign of the winding number. After a $2 \pi$ increase in the flux, the spectrum returns back to its original shape with $\Phi=0$.

This shifting of edge states in the energy spectrum can easily be probed using transmission spectrum measurements in our devices. Fig. 6.4(a, c) shows the measured and simulated transmission spectra as a function of external flux $\Phi$ and frequency $\omega$, for a square annulus with outer edge of 10 site rings and inner edge of 4 site rings (Fig. 6.1). Individual edge states can be resolved in this plot and can be seen to move in opposite directions. For any given input field frequency, which in this case acts as the Fermi energy level, for a $2 \pi$ increase in the external phase $\Phi$, only one edge state crosses the Fermi level. This indicates that the winding number 
of these edge states is \pm 1 , as expected.

Comparing Fig. 6.4(a, c) to Fig. 6.3(a), we see that the contour plot here shows only the outer edge states. This is because the probe waveguides are coupled only to the outer edge and the coupling to the inner edge decreases exponentially as $e^{-d / l_{0}}$, where $d$ is the number of rings between the outer and the inner edge (in this case 4) and $l_{0}=1 / \sqrt{2 \pi \alpha_{\mathrm{M}}}$ is the magnetic length in units of lattice spacing. Furthermore, the input/output coupling $\left(\kappa_{\mathrm{ex}}\right)$ to the annulus was made weak $\left(\kappa_{\mathrm{ex}}<4 J / N_{x} N_{y}\right)$, so as to resolve the individual peaks in the spectrum arising from transmission through the edge states.

Fig. 6.4(b) shows the overlap of the observed transmission spectra at $\frac{\Phi}{2 \pi}=$ $0.5,1.5$. We see that the two measured spectra roughly overlap and show qualitative agreement with the simulations. The discrepancy in the overlap is because we have heated only the outermost link rings for these measurements, even though we fabricated heaters on all the link rings as shown in Fig. 6.1. In our design, the heaters are placed $600 \mathrm{~nm}$ above the link ring waveguides. Therefore, instead of just heating the waveguides beneath them, the heaters also heat the neighboring rings and hence add to disorder in the lattice. When we tried heating all the link rings, we saw that the spectra distort significantly, making it impossible to resolve the edge states. We find that for $\Phi=0.5-1.5$, the spectrum retains its shape when only the outer loop is heated. Moreover, the small curvature observed in the shifting of edge states is also a result of the resonance frequency shift of the site rings (in the outer loop, connected by heated link rings) caused by heating the link rings. We have accounted for this disorder in the simulation. This technique of heating only 


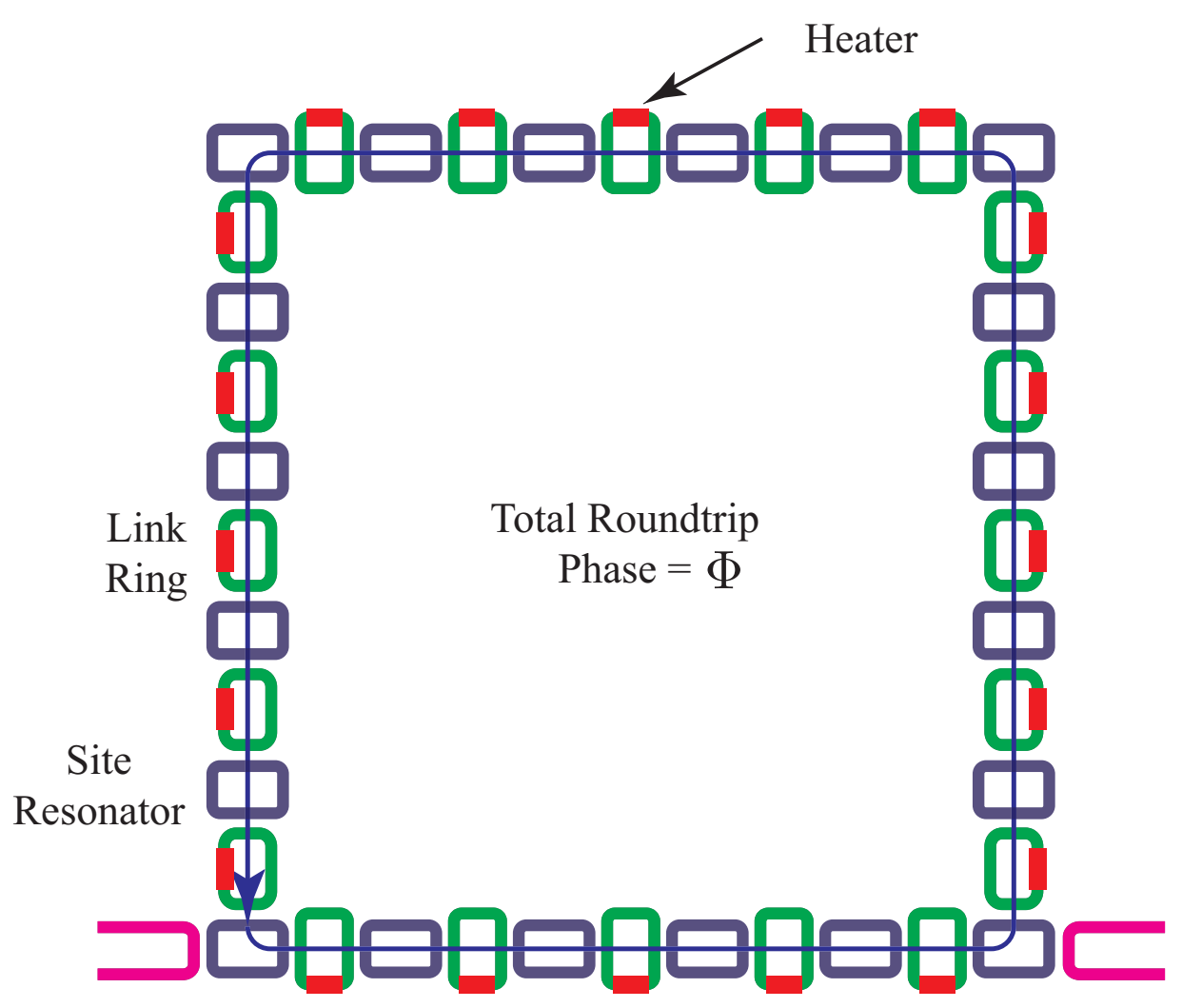

Figure 6.5: A ring geometry to investigate winding number of topological, but not robust, states. The ring supports $\mathrm{CW}$ and $\mathrm{CCW}$ circulating modes. External flux can be introduced for these states using the heaters.

the outer loop works mainly because of the fact that the edge states are confined to the edge of the annulus, which experiences the external gauge field. However, because of disorder in the lattice, the edge state might not always be confined to the edge but instead it may occupy some of the bulk. This, therefore, explains the only approximate overlap of the spectra after incurring a phase $\Delta \Phi=2 \pi$. We have also confirmed that heating only the inner edge does not shift the edge states. 


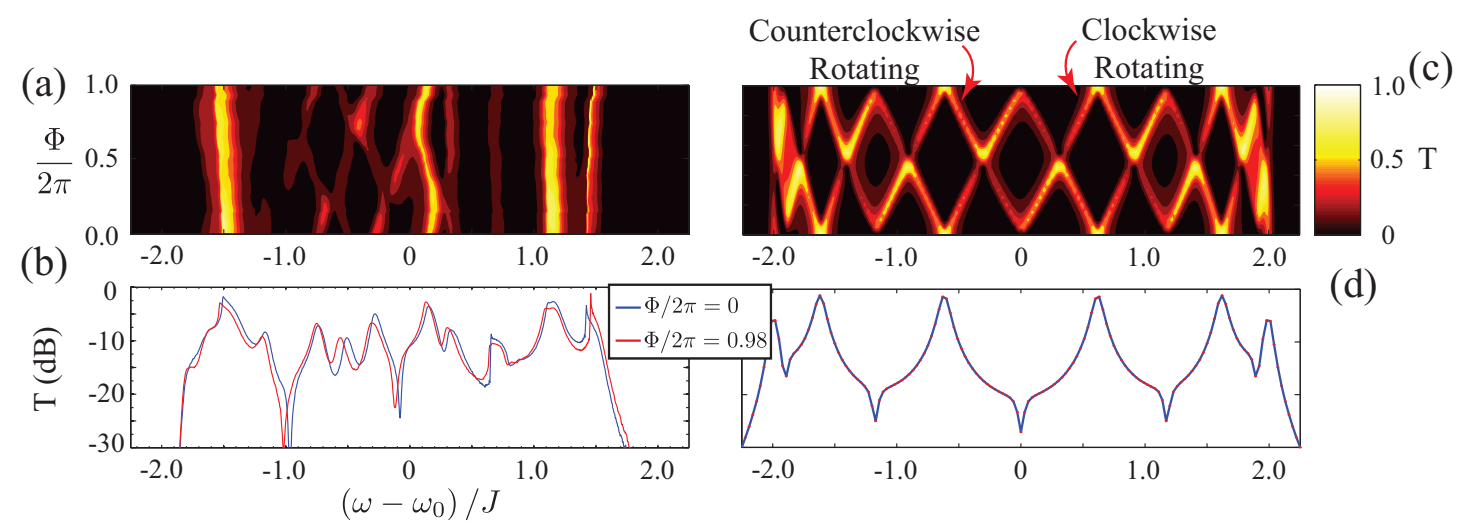

Figure 6.6: (a) Measured and (c) simulated transmission as a function of external flux $\Phi$ and frequency $\omega$. The CW and CCW modes move in opposite directions and cross the neighboring states. For one unit increase in flux, they move by exactly one position. Not all the crossings are observed in the experiments, because of the disorder in the ring. (b) Measured and (d) simulated transmission spectra at $\Phi=0,2 \pi$. Even though not all of the crossings are visible, the measured spectra overlap well. The significantly different shape of the measured transmission spectra compared to the simulations, indicates the severity of disorder. The measured spectrum at $\Phi=2 \pi$ has been scaled along the frequency axis (by a factor of 0.98), to offset the dispersion effect which results in slight broadening of the spectrum.

\subsection{Winding Number in a Chain of Ring Resonators}

We further investigate topological states in a chain of ring resonators (Fig. 6.5). This chain of rings is similar to the $1 \mathrm{D}$ array of rings, the CROW, which we discussed earlier, except for the coupling of the end rings. This geometry supports CW and CCW circulating modes around the chain. However, this system does not have any magnetic field. These states are therefore, in general, degenerate and thus are not robust against disorder. Fabrication-induced disorder can easily break the degeneracy of the two modes.

We introduce external flux into this system, as we did for the case of square annulus, by heating the link rings. Fig. 6.6 shows the experimentally observed and 
the simulated transmission as a function of magnetic flux $\Phi$ and frequency $\omega$. The additional phase lifts the degeneracy of the CW and the CCW states. With an increase in the external flux, these states move in opposite directions and cross the neighboring states. The states move by exactly one unit, indicating the winding number of these states. After a $2 \pi$ increase in the external flux, the transmission spectrum returns to its original shape (Fig. 6.6). We can however, only see some of the crossings in the experimental data. This is because of the disorder in the system which destroys the degeneracy of the CW and CCW modes, as is evident by the fact that crossings are visible for $\Phi$ other than integer multiples of $\pi$. Another indication is the big difference between the observed transmission spectrum and the simulation. This is contrary to the case of square annulus where the transmission spectra of the robust edge states qualitatively matched with the simulated spectrum. We do note, however, that even in the presence of disorder, the spectrum for $\Phi=2 \pi$ has excellent agreement with the spectrum at $\Phi=0$.

\subsection{Heater Calibration}

We used an ADF with a heater, to calibrate the phase shift acquired for a unit heater power. The ADF ring had exactly the same dimensions as the rings in the square annulus and the chain of resonators. Fig. 6.7 shows the observed phase shift as a function of heater power. The heater resistance was 120 Ohms and the voltage applied to the heater ranged from 0-3 Volts. We see that the acquired phase increases linearly with the heater power. 


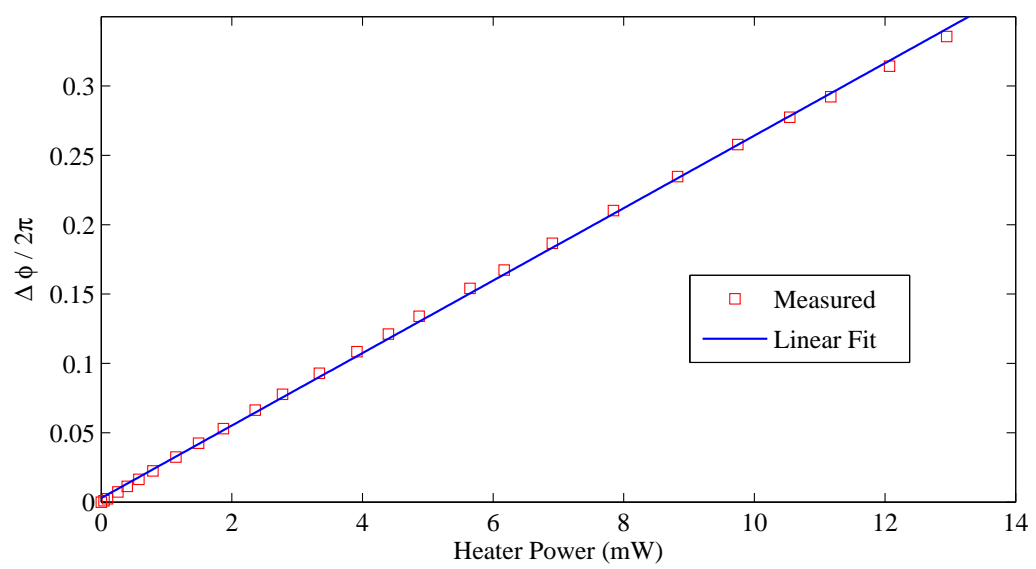

Figure 6.7: Measured phase shift in an ADF, as a function of the heater power. The acquired phase shift (in units of $2 \pi$ ) increases linearly with the heater power (in $\mathrm{mW}$ ), with a slope of 0.026(1).

For the square annulus and the chain geometry, we used this calibration curve to estimate $\Phi$ for a given voltage applied to the heaters. For the annulus, all the 12 heaters in a given loop (Fig. 6.1) were serially connected. The measured resistance was $1.4 \mathrm{KOhms}$ and the applied heater voltage across the loop ranged from $\approx 8-14$ Volts, which introduced the phase $\Phi=0.5 \pi-1.5 \pi$. Similarly, in the chain of rings (Fig. 6.5), all the 20 heaters were connected serially, giving a total resistance of 2.31 KOhms. The total voltage applied across the heaters ranged from $\approx 0-13$ Volts, for $\Phi=0-2 \pi$.

Furthermore, the transfer matrix analysis of a three-ring device (a link ring sandwiched between two site ring resonators) shows that if the heater introduces an extra phase $\Phi$ in one of the arms, the effective hopping phase (using CMT) between the link rings is $\Phi / 2$. The phase $\Phi$ used in preceding discussion of the annulus and the chain geometry is the effective hopping phase. Therefore, both the CW and the CCW propagating states (the long-edge and the short-edge states) enclose same 
additional phase.

\subsection{Summary}

In this Chapter, we demonstrated direct measurement of the winding number of the edge states, an integer characterizing the topological order. In the absence of conductance measurements in photonic systems, we employed Laughlin's adiabatic pump technique to shift the edge states in the energy spectrum using an external gauge field. Specifically, we used a square annulus geometry and used the thermo-optic effect in silicon to introduce the additional phase. We demonstrated the quantization of magnetic flux in photonic quantum Hall systems, which is analogous to quantization of conductance in electronic systems. We further compared this system to a simple ring geometry where the allowed eigenstates are topological but not robust. Even though our technique reveals the winding number in this geometry, the susceptibility of the system to fabrication induced disorders was clearly visible in the transmission spectrum measurements. 


\section{Chapter 7: Effect of Silicon Nonlinearities on Edge States}

At telecom wavelengths, silicon exhibits a plethora of nonlinear effects, for example, the Kerr effect, two-photon absorption (TPA), free carrier dispersion (FCD), free carrier absorption, etc. $[63,92-95]$. Because the refractive index of silicon is very high, light can be tightly confined in Si nanowaveguides which enhances the light intensity and ultimately the nonlinear effects in the waveguide. [63,96-98]. Additionally, using a ring resonator with high finesse further boosts the light intensity in the waveguide and also the length of interaction. Nonlinear processes in silicon waveguides and ring resonators have been used to generate entangled photons pairs $[99,100]$, heralded single photons [101], four-wave mixing [102-105], modulators [106], passive optical diodes [107] and for many other interesting and useful applications. Having used silicon ring resonators to implement topological photonic structures, it is a natural progression for us to investigate the effect of nonlinear interactions on the edge and bulk state transport. Furthermore, the edge-state transport could be used for robust, travelling wave optical wavelength conversion, as has been demonstrated in 1D array of coupled ring resonators [103].

In this Chapter, we develop a simple model to study the nonlinear effects in a silicon ring resonator using the single-mode approximation. Using an ADF and the 
pump-probe technique, we characterize the free parameters of our model. We then explore the effect of a strong pump on the transmission of edge states.

\subsection{Nonlinear Effects in Silicon Ring Resonators}

Silicon is a centro-symmetric crystal and, therefore, it does not support any second order nonlinear effects $\left(\chi^{2}\right)$ such as sum and difference frequency generation. However, the third order nonlinear term $\chi^{3}$ is non-zero. This is the basis of the Kerr nonlinearity and Two Photon absorption (TPA) found in silicon. Moreover, since silicon is a semiconductor, TPA leads to a generation of excess free carriers (electron and hole pairs) which affect the refractive index (Free Carrier Dispersion - FCD) and also absorb incident power (Free Carrier Absorption - FCA). TPA and FCA also result in heating of the device which ultimately leads to a refractive index change through the Thermo-Optic(TO) effect.

For a ring resonator, the light intensity in the ring waveguide and, therefore, the nonlinear effects are enhanced by the finesse of the resonator. To model these enhanced nonlinear effects in a silicon ring resonator, we use the single-mode approximation where the time evolution of the ring energy amplitude $a$ is given as (see Eq. 3.17)

$$
\frac{d a}{d t}=i\left(\omega-\omega_{0}\right) a+\left(2 \kappa_{\mathrm{ex}}+\kappa_{\mathrm{in}}\right) a-\sqrt{2 \kappa_{\mathrm{ex}}} \varepsilon_{\mathrm{in}}
$$

and the steady-state solution for the energy amplitude is

$$
a=\frac{\sqrt{2 \kappa_{\mathrm{ex}}} \varepsilon_{\mathrm{in}}}{i\left(\omega-\omega_{0}\right)+\left(2 \kappa_{\mathrm{ex}}+\kappa_{\mathrm{in}}\right)} .
$$

The resonance frequency $\omega_{0}$ of the ring is a function of the effective refractive index 
$\left(n_{\text {eff }}\right)$ of light in the ring waveguide and a change $\Delta n_{\mathrm{eff}}$ in this index results in resonance frequency shift $\Delta \omega_{0}$ as

$$
\Delta \omega_{0}=-\frac{\omega_{0}}{n_{\mathrm{eff}}} \Delta n_{\mathrm{eff}} \approx-\frac{\omega_{0}}{n_{\mathrm{eff}}} \Delta n
$$

where $\Delta n$ is the change in the bulk refractive index of silicon. The resonator loss rate $\kappa_{\text {in }}$ is related to the waveguide loss coefficient $\alpha$ as

$$
\kappa_{\text {in }}=\alpha v_{\mathrm{g}}
$$

The nonlinear absorption mechanisms, TPA and FCA, increase the waveguide loss coefficient by $\Delta \alpha$. The corresponding change, $\Delta \kappa_{\text {in }}$, in resonator loss rate is then,

$$
\Delta \kappa_{\mathrm{in}}=\Delta \alpha v_{\mathrm{g}}
$$

Furthermore, the nonlinear refractive index contributions are very small compared to $n_{\mathrm{eff}}$ and we can, therefore, assume that the nonlinear effects do not change $\kappa_{\mathrm{ex}}$. Thus, in principle, a knowledge of $\Delta \omega_{0}$ and $\Delta \kappa_{\text {in }}$ as a function of intensity in the ring waveguide is sufficient to analyze the effect of nonlinear processes on a ring resonator. In the following, we derive expressions for $\Delta \omega_{0}$ and $\Delta \kappa_{\text {in }}$ due to various nonlinear processes. Our analysis assumes that the system is in steady state, i.e., it is valid for time scales much longer than the carrier lifetime and the heat lifetime in the resonator.

\subsubsection{Kerr Effect}

The Kerr effect is due to the nonlinear response of bound electrons and causes a refractive index change which is proportional to the intensity $I_{\mathrm{R}}$ of the field present 
in the ring waveguide, i.e.

$$
\Delta n^{\mathrm{Kerr}}=n_{2}^{\mathrm{Kerr}} I
$$

The reported Kerr coefficient $n_{2}^{\text {Kerr }}$ in silicon is $4.4 \times 10^{-18} \mathrm{~m}^{2} \mathrm{~W}^{-1}$ [96]. For a ring resonator,

$$
I=\frac{P_{\mathrm{R}}}{A}=|a|^{2} \frac{v_{\mathrm{g}}}{A L}
$$

where $P_{\mathrm{R}}$ is the field power in the ring waveguide with cross section area $A$ and $L$ is the ring length. Using this relation, we can express the Kerr refractive index change as

$$
\Delta n^{\text {Kerr }}=n_{2}^{\text {Kerr }}|a|^{2} \frac{v_{\mathrm{g}}}{V}
$$

Here, $V=A L$ is the ring volume.

\subsubsection{Two-Photon Absorption}

The bandgap of silicon is $\approx 1.1 \mathrm{eV}$ and, thus, it has a linear absorption edge at about $1.2 \mu \mathrm{m}$. However, because of the third order nonlinearity, two photons with a wavelength $>1.2 \mu \mathrm{m}$ can still be simultaneously absorbed as long as the combined energy of both the photons is sufficient to overcome the bandgap. This TPA process leads to absorption of input light where the absorption coefficient itself is proportional to the light intensity in the ring waveguide. The two-photon absorption loss coefficient in the waveguide is given as

$$
\alpha_{\mathrm{TPA}}=\beta_{2} I
$$


where $\beta_{2}$ is the TPA coefficient and is $8.4 \times 10^{-12} \mathrm{~mW}^{-1}$ [96]. The additional resonator loss rate $\Delta \kappa_{\text {in }}^{\mathrm{TPA}}$ due to TPA is therefore

$$
\Delta \kappa_{\mathrm{in}}^{\mathrm{TPA}}=\beta_{2}|a|^{2} \frac{v_{\mathrm{g}}^{2}}{V}
$$

where we have used Eqs. 7.5 and 7.9. The total power absorbed in the ring due to TPA can be calculated as

$$
P_{\mathrm{abs}}^{\mathrm{TPA}}=2 \Delta \kappa_{\mathrm{in}}^{\mathrm{TPA}}|a|^{2}=2 \beta_{2}|a|^{4} \frac{v_{\mathrm{g}}^{2}}{V}
$$

\subsubsection{Free Carriers}

Since silicon is a semiconductor, TPA leads to generation of free carriers, electrons and holes, in the conduction and valence bands, respectively. The generated free carrier number density (both for electrons and holes) is given as

$$
N_{\mathrm{c}}=\frac{P_{\mathrm{abs}}^{\mathrm{TPA}} \tau_{c}}{2 \hbar \omega V}=\beta_{2}|a|^{4} \frac{\tau_{c}}{\hbar \omega} \frac{v_{\mathrm{g}}^{2}}{V^{2}}
$$

where $\tau_{c}$ is the free carrier lifetime in silicon. Note that the carrier lifetime in a silicon waveguide is significantly different than that in bulk silicon and it also depends on the waveguide geometry and the fabrication technology. The reported values for carrier lifetime in silicon waveguides vary from $\approx 1$ ns to over $200 \mathrm{~ns}$. This is mainly because of the carrier recombination at the rough waveguide surface which lowers their lifetime. Moreover, the carrier lifetime is a function of the free carriers density and can be modelled as

$$
\tau=\frac{1}{A+B N_{\mathrm{c}}{ }^{r}}
$$


where the parameters $A, B$ and $r$ depend on waveguide geometry and are generally treated as fitting parameters.

If the generated free carriers contribute an absorption cross-section of $\sigma_{\mathrm{FCA}}$ per unit carrier density, the total increase in the absorption coefficient of the ring waveguide is

$$
\alpha_{\mathrm{FCA}}=\sigma_{\mathrm{FCA}} N_{\mathrm{c}}
$$

and the corresponding increase in the resonator loss rate is

$$
\Delta \kappa_{\mathrm{in}}^{\mathrm{FCA}}=\alpha_{\mathrm{FCA}} v_{\mathrm{g}}=\sigma_{\mathrm{FCA}} \beta_{2}|a|^{4} \frac{\tau_{c}}{\hbar \omega} \frac{v_{\mathrm{g}}^{3}}{V^{2}}
$$

The observed absorption cross-section $\sigma_{\mathrm{FCA}}$ for silicon is $14.5 \times 10^{-22} \mathrm{~m}^{2}$ [96]. The total absorbed power in the ring resonator due to FCA is given as

$$
P_{\mathrm{abs}}^{\mathrm{FCA}}=2 \Delta \kappa_{\mathrm{in}}^{\mathrm{FCA}}|a|^{2}=2 \sigma_{\mathrm{FCA}} \beta_{2}|a|^{6} \frac{\tau_{c}}{\hbar \omega} \frac{v_{\mathrm{g}}^{3}}{V^{2}}
$$

As evident, the FCA power absorption is proportional to $I^{3}$ and is, therefore, the most significant nonlinear process at high ring intensities.

The generated free carriers also lead to dispersion, i.e., a change in the refractive index which can be calculated as,

$$
\Delta n^{\mathrm{FCD}}=-\zeta N_{\mathrm{c}}=-\zeta \beta_{2}|a|^{4} \frac{\tau_{c}}{\hbar \omega} \frac{v_{\mathrm{g}}^{2}}{V^{2}}
$$

Here, $\zeta=8.8(4.6) \times 10^{-28} \mathrm{~m}^{3}$ for electrons(holes) is the change in refractive index per unit free carrier density. The negative sign indicates that an increase in the carrier density reduces the refractive index of the ring waveguide. 


\subsubsection{Thermo-Optic Effects}

Absorption of power in the ring waveguide heats the waveguide which changes its refractive index through Thermo-Optic (TO) effect. This change in refractive index due to thermal effects is written as

$$
\Delta n=\frac{d n}{d T} \frac{d T}{d P_{\mathrm{abs}}} P_{\mathrm{abs}}
$$

Here, $\frac{d n}{d T}=1.86 \times 10^{-4} \mathrm{~K}^{-1}$ is the thermo-optic coefficient of silicon. $\frac{d T}{d P_{\text {abs }}}$ is the change in temperature of the waveguide for a unit of absorbed power and it depends on the material and shape of the waveguide, top and bottom cladding layers and also the substrate. Reported values of $\frac{d T}{d P_{\mathrm{abs}}}$ in silicon waveguides range from 5 to $40 \mathrm{~K} / \mathrm{mW}[96] . P_{\text {abs }}$ is the total absorbed power in the ring waveguide due to linear absorption, TPA and FCA. It can be evaluated as

$$
P_{\mathrm{abs}}=P_{\mathrm{abs}}^{\mathrm{Lin}}+P_{\mathrm{abs}}^{\mathrm{TPA}}+P_{\mathrm{abs}}^{\mathrm{FCA}}=2 \gamma \kappa_{\mathrm{in}}|a|^{2}+2 \beta_{2}|a|^{4} \frac{v_{\mathrm{g}}^{2}}{V}+2 \sigma_{\mathrm{FCA}} \beta_{2}|a|^{6} \frac{\tau_{c}}{\hbar \omega} \frac{v_{\mathrm{g}}^{3}}{V^{2}},
$$

where $\gamma$ is the fraction of linear waveguide loss which is due to absorption in silicon (and not scattering) and hence, contributes to heating. For bulk silicon, the linear material absorption coefficient at telecom wavelengths is negligible. However, for silicon waveguides, the surface roughness leads to a finite linear absorption coefficient estimated to be $\approx 10-40 \%$ of the total linear loss coefficient $\alpha$.

\subsubsection{Modeling}

Having discussed the various nonlinear effects, we now proceed to further simplify our model for the ring resonator. We write the total shift in the ring resonance 
frequency as

$$
\Delta \omega_{0}=-\frac{\omega_{0}}{n_{\mathrm{eff}}} \Delta n=-\frac{\omega_{0}}{n_{\mathrm{eff}}}\left(N_{2}|a|^{2}+N_{3}|a|^{4}+N_{4}|a|^{6}\right)
$$

The refractive index parameters, $N_{2}, N_{3}, N_{4}$, are first, second and third order terms in the ring energy $|a|^{2}$, respectively. From the preceding discussion, we see that $N_{2}$ represents the combined Kerr nonlinearity and TO effect due to linear absorption. The FCD and the TO effect due to TPA are contained in $N_{3} . N_{4}$ is the refractive index change caused by the TO effect related to free-carrier absorption. Thus,

$$
\begin{aligned}
N_{2} & =n_{2}^{\mathrm{Kerr}} \frac{v_{\mathrm{g}}}{V}+2 \frac{d n}{d T} \frac{d T}{d P_{\mathrm{abs}}} \gamma \kappa_{\mathrm{in}} \\
N_{3} & =-\zeta \beta_{2} \frac{\tau_{c}}{\hbar \omega} \frac{v_{\mathrm{g}}^{2}}{V^{2}}+2 \frac{d n}{d T} \frac{d T}{d P_{\mathrm{abs}}} \beta_{2} \frac{v_{\mathrm{g}}^{2}}{V} \\
N_{4} & =2 \frac{d n}{d T} \frac{d T}{d P_{\mathrm{abs}}} \sigma_{\mathrm{FCA}} \beta_{2} \frac{\tau_{c}}{\hbar \omega} \frac{v_{\mathrm{g}}^{3}}{V^{2}} .
\end{aligned}
$$

Similarly, we can write the total change in resonator loss rate $\Delta \kappa_{\text {in }}$ as

$$
\Delta \kappa_{\text {in }}=v_{\mathrm{g}}\left(B_{2}|a|^{2}+B_{3}|a|^{4}\right)
$$

where $B_{2}$ and $B_{3}$ are the waveguide loss parameters due to TPA and FCA, respectively. They are first and second order in ring energy, respectively, and

$$
\begin{aligned}
B_{2} & =\beta_{2} \frac{v_{\mathrm{g}}}{V} \\
B_{3} & =\sigma_{\mathrm{FCA}} \beta_{2} \frac{\tau_{c}}{\hbar \omega} \frac{v_{\mathrm{g}}^{2}}{V^{2}} .
\end{aligned}
$$

Using these relations and (7.2), we can write the steady state solution for the ring energy amplitude, in the presence of nonlinear effects, as $a=\frac{-i \sqrt{2 \kappa_{\mathrm{ex}}} \varepsilon_{\mathrm{in}}}{i\left(\omega-\omega_{0}+\frac{\omega_{0}}{n_{\mathrm{eff}}}\left(N_{2}|a|^{2}+N_{3}|a|^{4}+N_{4}|a|^{6}\right)+\left(2 \kappa_{\mathrm{ex}}+\kappa_{\mathrm{in}}+v_{\mathrm{g}}\left(B_{2}|a|^{2}+B_{3}|a|^{4}\right)\right)\right.}$. 
However, an analytic solution for the ring energy amplitude is not possible using this equation. Nevertheless, it can be solved using numerical methods (for example, the iterative technique) to estimate $a$ and hence, the transmission at the drop and through ports.

\subsection{Experimental Characterization of Nonlinear Parameters}

We use an ADF to characterize the nonlinear effects in our system. For an $\mathrm{ADF}$, a change in refractive index leads to a shift in the resonance frequency (or the transmission spectrum) of the resonator as

$$
\Delta \omega_{0}=\frac{\omega_{0}}{N_{\text {eff }}} \Delta n
$$

and an increase in the loss rate, due to TPA and FCA, results in a change in the through port transmission contrast $C_{\mathrm{T}}$ as

$$
\frac{1}{\sqrt{C_{\mathrm{T}}}}=\frac{\kappa_{\mathrm{in}}+\Delta \kappa_{\mathrm{in}}}{2 \kappa_{\mathrm{ex}}}
$$

By measuring the shift in resonance frequency (of the transmission spectrum) and the through port contrast as a function of ring intensity, it is possible to extract the nonlinear parameters using the expressions derived in the previous section.

However, an accurate and simultaneous experimental estimation of all the nonlinear effects in silicon is extremely challenging, mainly because of the waveguide geometry dependence of the free carrier dynamics and the thermal effects. We, therefore, assume that the material parameters such as the TPA coefficient $\left(\beta_{2}\right)$, the FCA cross-section $\left(\sigma_{\mathrm{FCA}}\right)$, the refractive index change due to free carriers $(\zeta)$ 


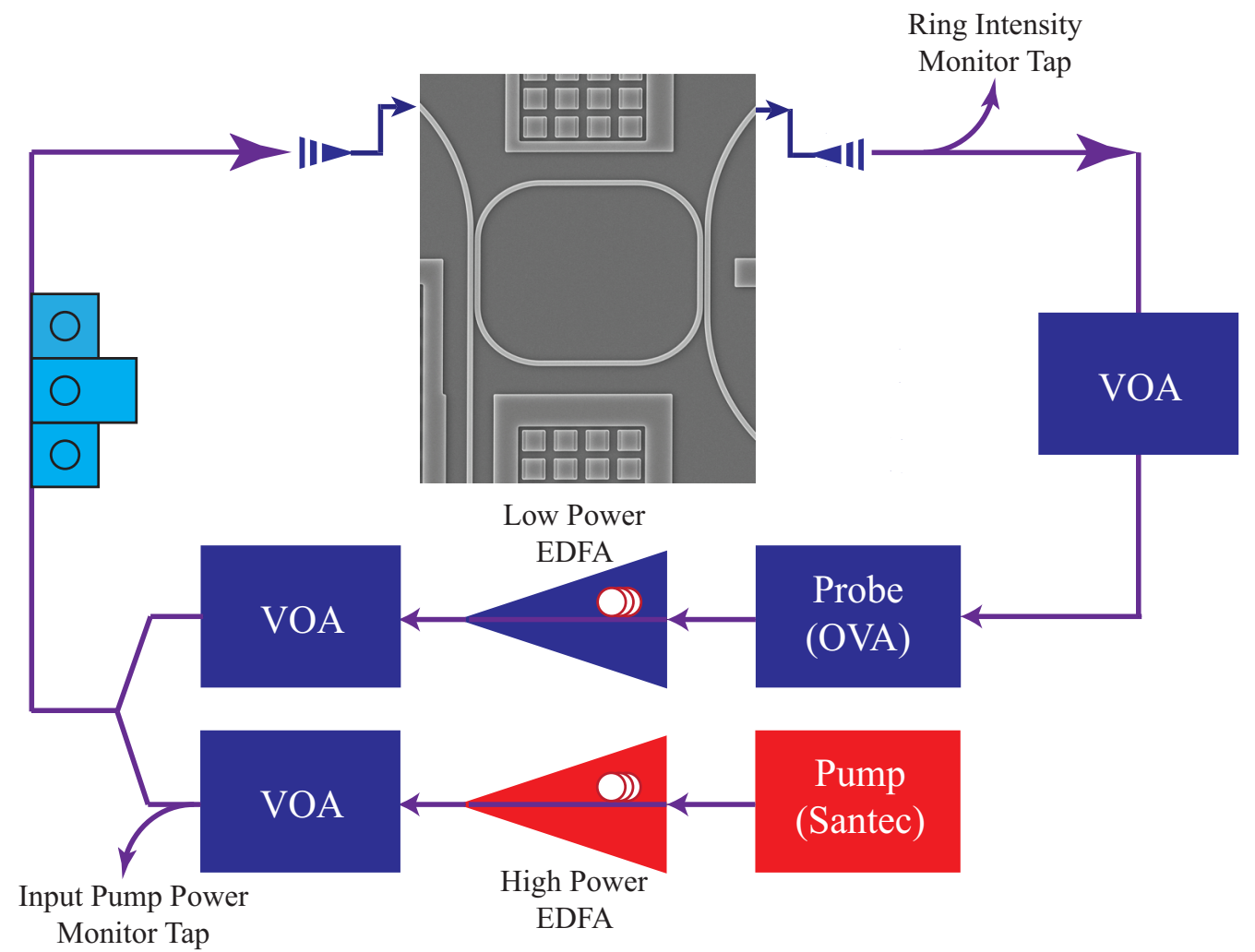

Figure 7.1: Pump-probe setup to investigate the nonlinear effects in the coupled ring resonator structures.

and the TO coefficient $\left(\frac{d n}{d T}\right)$ are known. We assume that the other parameters such as $N_{\text {eff }}, v_{\mathrm{g}}, \kappa_{\mathrm{ex}}, \kappa_{\mathrm{in}}, V, L$ are also known. Then, the quantities $N_{2}, N_{3}, N_{4}, B_{2}$ and $B_{3}$ are in fact functions of only three free parameters - the carrier lifetime $\tau$, the change in temperature of the waveguide per unit absorbed power $\frac{d T}{d P_{\mathrm{abs}}}$ and the fraction $\gamma$ of the linear loss rate $\alpha$ which is due to absorption. These free parameters are waveguide geometry dependent and we use them as fitting variables.

We use a pump-probe setup to estimate the free parameters of our model. The high power pump sets the nonlinearity in the ring. The nonlinear response of the ring is analyzed by measuring the transmission spectrum (at through and drop ports) of the ring using a weak probe signal with tunable wavelength (LUNA OVA). 

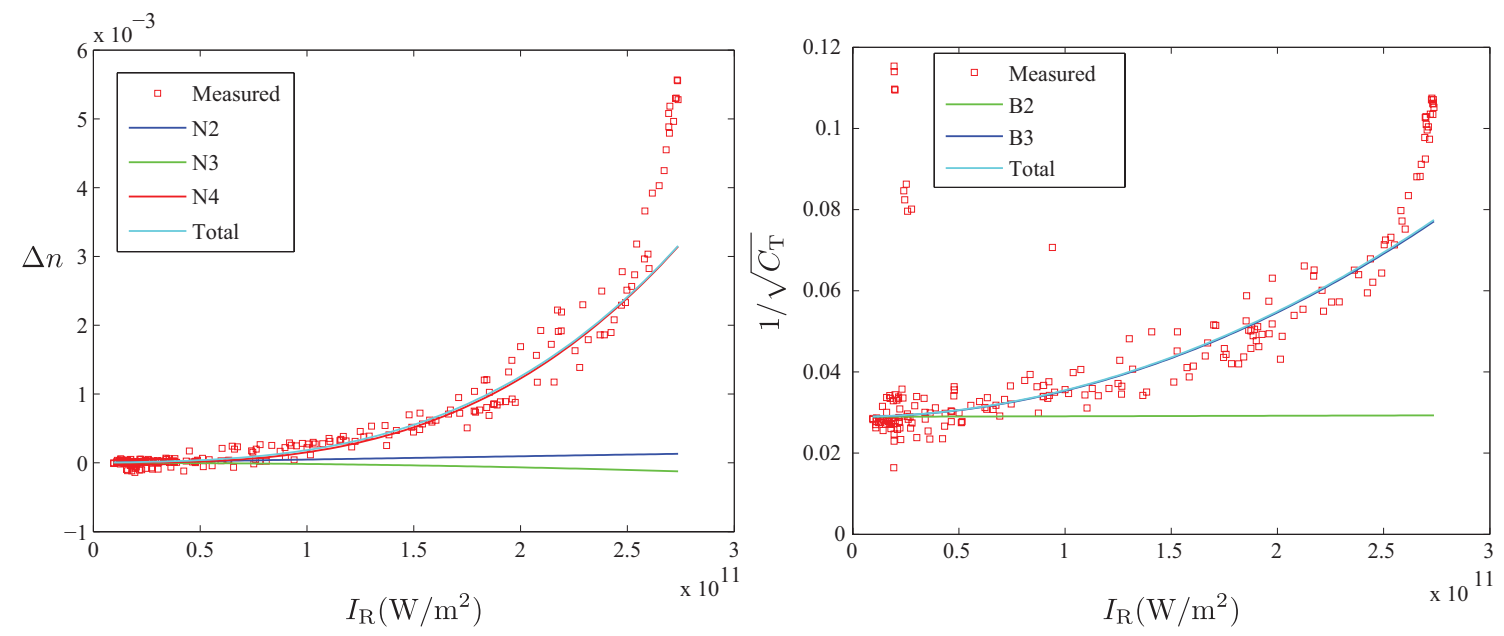

Figure 7.2: (a) Measured change in refractive index as a function of ring intensity. Contributions to $\Delta n$ from $N_{2}$ (blue), $N_{3}$ (green) and $N_{4}$ (red), calculated using the fitted parameters are also shown. (b) Measured inverse square root of the through port contrast as a function of ring intensity. The plot also shows the respective contributions by $B_{2}$ (green) and $B_{3}$ (blue) nonlinear processes. The most dominant mechanism is the FCA.

To vary the light intensity in the ring, we sweep the frequency of the pump with respect to the ring resonance frequency. The pump frequency is set in one FSR of the spectrum and the probe in the neighboring FSR. The pump power travelling in the ring is then governed by Eq. 7.27, which requires a knowledge of the parameters we seek. Since $\mathcal{E}_{\mathrm{D}}=\sqrt{2 \kappa_{\mathrm{ex}}} a$, we can use the measured transmission at the drop port to estimate the pump energy in the ring.

Fig. 7.2 shows the measured resonance shift and contrast as a function of ring intensity. The data is taken across 2 different devices, for 3 different input pump power levels. A fit to the shift and contrast data give $\tau_{\mathrm{c}}=44 \mathrm{~ns}, \frac{d T}{d P_{\mathrm{abs}}}=12.4 \mathrm{~K} / \mathrm{mW}$ and $\gamma$ to be 0.07 . The observed carrier lifetime in our system is more than an order of magnitude higher than that reported for similar waveguide geometries [96]. We are uncertain of the reason for this discrepancy. It could be due to global heating 
of the chip which we see in our system. We observe that apart from the local shift of the ADF resonance due to thermal effects, the chip is globally heated because of the high laser power incident on the input grating coupler (Section 7.3). Moreover, in our model, we have not considered the spatial and temporal dynamics of the free carriers and the thermal effects. For simplicity, we have assumed a steady state value for the carrier density and the temperature. The reported carrier lifetime $(\approx \mathrm{ns})$ and the heat lifetime $(\approx \mu \mathrm{s})$ are orders of magnitude higher than the photon lifetime in our low finesse ring resonators $(\approx \mathrm{ps})$. Developing a model which incorporates all these dynamics is extremely challenging and computationally intensive, especially for the 2D lattice of coupled resonators.

We also observe that the fit to the measured data is particularly bad at high ring intensities. At high ring intensities, the observed shift in the resonance spectrum is about one half of the FSR. Such a large shift suggests the breakdown of the singlemode approximation in this regime. Transfer matrix analysis for the nonlinear ring resonator might avoid some of these issues. A first step would be to write the differential equation for the propagation of field inside the ring as

$$
\frac{d \mathcal{E}(z)}{d z}=\left(i \frac{\omega}{c} n(z)-\alpha(z)\right) \mathcal{E}(z)
$$

where $z$ is the length along the ring waveguide, $n(z)$ and $\alpha(z)$ are the effective refractive index and absorption coefficient, respectively. This equation along with 
the boundary conditions

$$
\begin{aligned}
\mathcal{E}(0) & =i \kappa \mathcal{E}_{\text {in }}+t \mathcal{E}(L) \\
\mathcal{E}\left(\frac{L}{2}+\epsilon\right) & =t \mathcal{E}\left(\frac{L}{2}\right) \\
\mathcal{E}_{\mathrm{D}} & =i \kappa \mathcal{E}\left(\frac{L}{2}\right) \\
\mathcal{E}_{\mathrm{T}} & =t \mathcal{E}_{\text {in }}+i \kappa \mathcal{E}(L),
\end{aligned}
$$

give a solution for the field inside the ring. Here, we have assumed that the input and output waveguides are coupled to the ring at $z=0$ and $z=L / 2$, respectively. However, this procedure would be very difficult to implement for the lattice of ring resonators.

\subsection{Problem of Global Shift}

In our experiments, we find that even when the pump is in the mid-FSR, i.e., when it does not enter the ring, it causes huge resonance shifts in the ADF spectrum (Fig. 7.3). Further, this shift is similar in magnitude to a shift induced by moving the fiber away from the grating coupler, so that most of the pump power doesn't couple into the silicon waveguide but just hits the silicon substrate. This shift is measured to be $0.11 \mathrm{GHz}$ per mW of pump power at the output of the EDFA. We call this shift the global thermal shift because it does not depend on the intensity of the pump power in the waveguide. It depends solely on the pump power incident on the chip. Therefore, for the characterization of nonlinear parameters in our system, we used a pump wavelength sweep to vary the pump intensity in the ring and fixed the input pump power to ensure that the global shift remained constant. With 

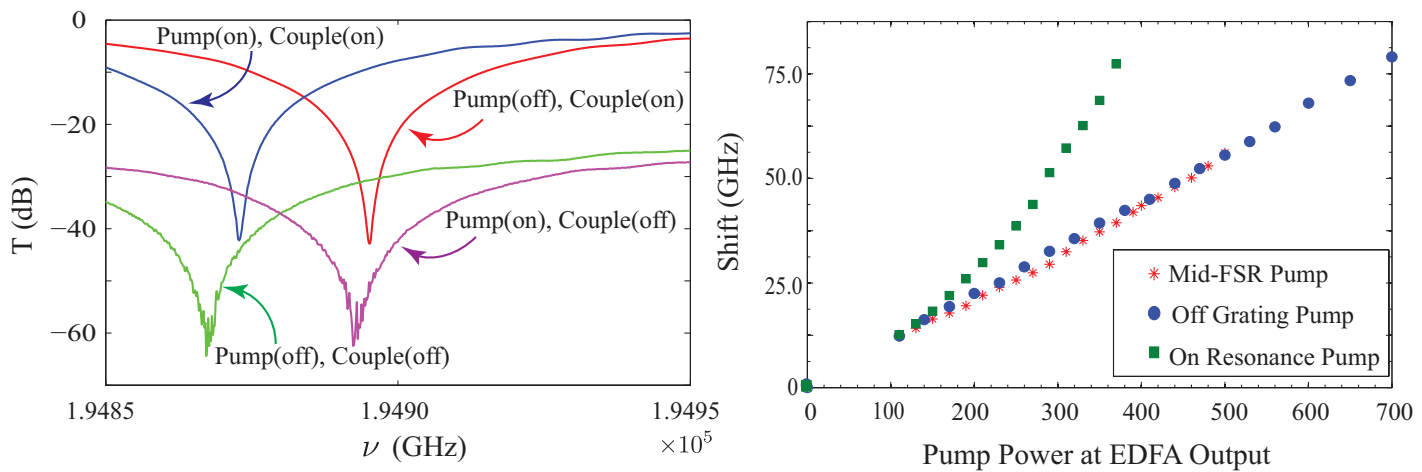

Figure 7.3: (a) Indication of global heating. The input light shining on any part of the chip results in almost the same shift as it does while passing through the coupling waveguide of the ADF (off-resonant pump). Pp-off/on refers to the pump power being off and on. Cp-on indicates the fiber is aligned on the grating coupler for maximum pump power coupling to the ADF. For Cp-off, the fiber is shifted away from the grating coupler, so that most of the pump power falls on the silicon substrate, with just a small fraction passing through the ADF to measure its response. (b) Global shift introduced as a function of the pump power incident on the chip. Off-resonant pump and a pump detuned from the grating coupler result in similar shifts.

this procedure, when the shift is calibrated to a transmission spectrum taken in the presence of a mid-FSR pump, the resulting shift is independent of global shift. This is not the case when we fix the pump frequency and vary the pump power instead. In that case, the shift arising from nonlinearity in the ring resonator is convoluted with the global shift.

For a 2D lattice of ring resonators, this global shift can lead to a uniform increase in temperature throughout the lattice and hence, wash out any changes in the transmission spectrum. However, we confirmed that along with a global shift, each ring also has a local heat/shift component and the lattice does not thermalize as a whole. We used a three-ring device - a link resonator sandwiched between two site resonators. Fig. 7.4 shows the observed spectra when the pump is at the resonance of site rings. The spectra have been shifted to align the peaks of the link 


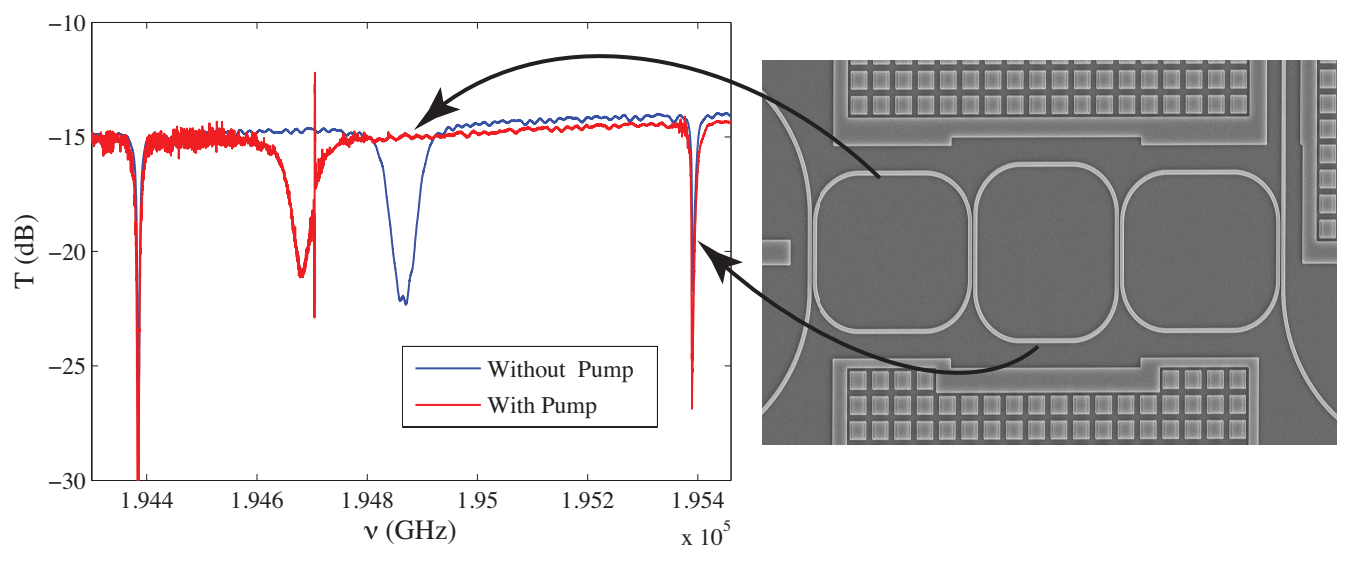

Figure 7.4: Through port transmission spectra of a three-ring device, a link resonator sandwiched between two site rings, with and without pump. The spectra have been shifted to align the transmission dip of the link ring, thus offsetting the global shift component. The transmission dips of the site rings show a shift, indicating a local component of heat which arises from the nonlinear interactions in the site rings.

resonator, which accounts for the global shift contribution (since the pump is at the resonance of site rings). We can clearly see that there is a differential shift in the site and link resonators. This implies that a fraction of the total nonlinear shift is in fact local to a single ring resonator and we can hope to investigate nonlinear effects in a $2 \mathrm{D}$ lattice.

\subsection{Effect of Nonlinear Interactions on Edge States}

To explore the effect of silicon nonlinearities on the topological edge states, we employed the pump-probe setup shown in Fig. 7.1 on a $8 \times 8$ lattice. The strong pump beam is tuned within one FSR and the transmission spectrum of the lattice is probed at a neighboring spectrum. We fixed the pump power and scanned the pump wavelength across the resonance spectrum of the lattice. We find that when the pump is positioned in the edge-state region of the spectrum, the spectrum of 

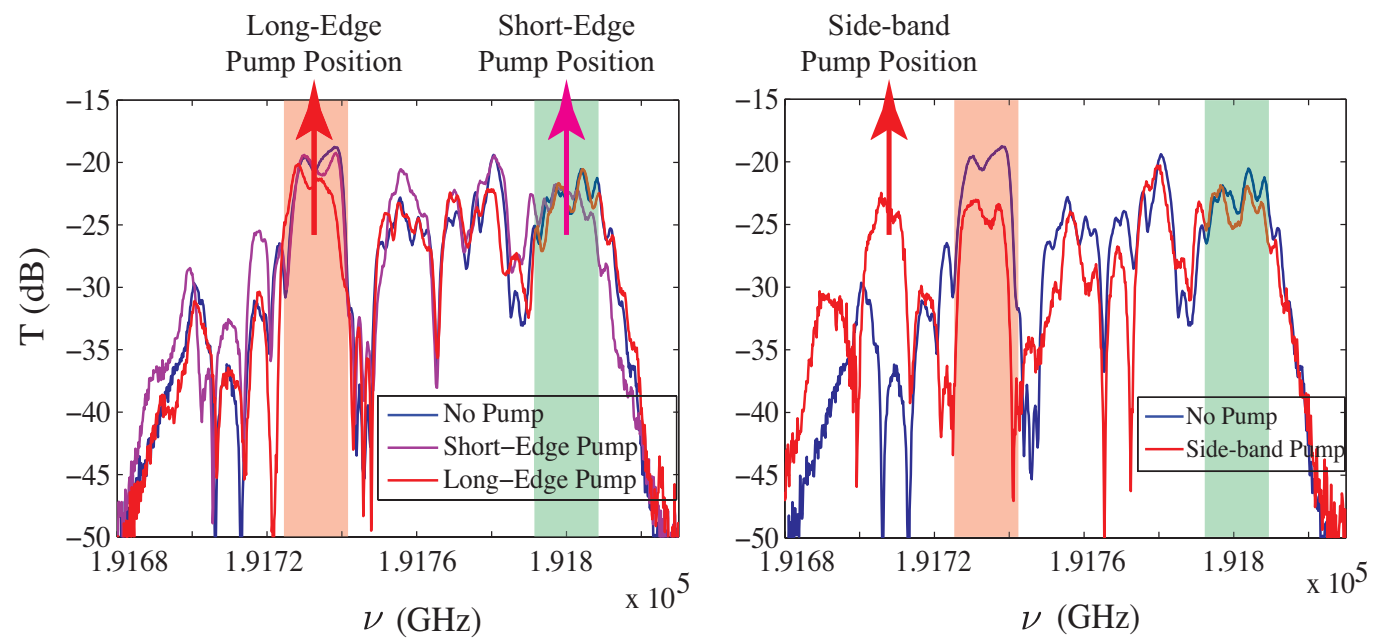

Figure 7.5: (a) Transmission spectra of a $8 \times 8$ lattice device, without a pump, and with a pump in the short (green) and long-edge (red) region. The shape of the spectrum does not change. (b) Transmission spectra when the pump is positioned in a side-band of the long-edge. The transmission in the long-edge region reduces by $\approx 5 \mathrm{~dB}$.

the lattice does not change in shape at all (Fig. 7.5(a)). However, when the pump is positioned in the neighboring regions, the edge state transmission is drastically reduced, by as much as $5 \mathrm{~dB}$. Simultaneously, the transmission at frequencies close to the pump rises by approximately the same amount.

We have tried to simulate this effect using the nonlinear model discussed above for a single ring. However, when we use a carrier lifetime of 44 ns in the model (from ADF data fitting), the transmission spectrum of the lattice is greatly reduced because of the very strong FCA. We therefore conclude that further investigation is required to understand this effect. The best way forward will be to use a pulsed pump and probe beams. By using a pulse width less than the heat lifetime $(\approx \mu \mathrm{s})$ and a repetition rate more than that, we can eliminate all of the thermo-optic effects as well as the global heating effects. In that case, the dominant nonlinear dispersion effect would only be the free carrier dispersion. This should result in a significant 
simplification of the model and hopefully, a simulation of the observed effect on edge states would be possible.

\subsection{Summary}

In this Chapter, we discussed the various nonlinear effects in a silicon resonator. We developed a simple model based on single-mode approximation. In this model, the nonlinear dispersive effects are captured by the shift in the resonance frequency and the absorptive effects are included as additional resonator loss rates. We find, however, that this simple model is insufficient to explain the observed change in refractive index and the loss rate, especially at high pump powers. Further, the observed free carrier lifetime is significantly higher than that reported in the literature for similar waveguide dimensions. In the $2 \mathrm{D}$ lattice of ring resonators, we find that a strong pump positioned in the edge state regions does not affect the spectrum, apart from a shift of the spectrum. However, when the pump is positioned in the side-bands of the long-edge, we see that the transmission in the long-edge state decreases by $\approx 5 \mathrm{~dB}$. We have not been able to simulate this effect. We conclude that further experiments, using a pulsed pump and probe beams, are required to investigate the the effect of nonlinear interactions on edge states. 


\section{Chapter 8: Conclusions and Outlook}

Large scale photonic integration demands an arbitrary control over the flow of photons, for example, unidirectional waveguides and reflection-free sharp bends. Bandgap engineering in photonic crystals and metamaterials has been widely used to manipulate the transport of photons. Using a magnetic field to control photonic transport, analogous to electronic systems, is another viable option. However, magneto-optic effect - the only source of coupling between a magnetic field and photons - is very weak at optical frequencies. At optical frequencies, it is feasible to introduce a synthetic magnetic field for photons. This work demonstrates the implementation of such a synthetic magnetic field using a two-dimensional lattice of coupled ring resonators, on a silicon photonics platform. Specifically, we implement photonic analogs of the topologically robust edge states which appear in quantum Hall systems.

We began with a discussion of the integer quantum Hall effect and the tightbinding lattice model generally used to study this effect. We showed that for a finite lattice, edge states emerge naturally in the energy spectrum of the system. These states are chiral, topologically protected and robust against lattice disorders. We then described the design of a two-dimensional lattice of ring resonators which 
implements a synthetic magnetic field for photons. The lattice site resonators were coupled using link rings and the magnetic field was introduced by vertically shifting the link rings. We used input-output formalism to simulate the transmission and delay spectra of the lattice. The presence of edge states was evident in the transport measurements. We further discussed an application of these edge states as robust optical delay lines.

We implemented this system using CMOS-compatible SOI technology and demonstrated the presence of edge state using direct imaging. We then presented first quantitative analysis of the robustness of edge states against fabrication induced disorder. Using transmission and delay distributions, we showed that the localization is suppressed in edge states. We measured the winding number of the edge states. The quantized Hall conductance in electronic systems manifests as quantization of winding number in photonic quantum Hall systems. Finally, we investigated the effect of nonlinear interactions on edge states. We observed that a strong pump can significantly affect the transmission through edge states. Our simple model was, however, not able to simulate this observation.

Our system provides a new platform to explore the various quantum Hall phenomena, at room temperature. For example, using the thermo-optic effect, as we did for the winding number measurements, it is possible to probe the Hofstadter butterfly spectrum - which has only been recently spotted using cold atoms and van der Waal heterostructures, and graphene [108-110]. Because photons do not interact with each other, the experimental efforts have been limited to the non-interacting regime, i.e., simulations of the integer quantum Hall effect. Adding photon-photon 
interactions to this system, for example using quantum dots, would further enable investigations of the intriguing fractional quantum Hall physics. Moreover, the experiments reported in this thesis used coherent laser source as input. It would be very interesting to study the transport of correlated photons through the lattice. Similar experiments in one-dimensional arrays of coupled waveguides have revealed non-trivial quantum correlations [111-113]. Our system, thus, opens a new avenue to investigate the transport of photons under the influence of a gauge field and use topological features to achieve new photonic device functionalities. 


\section{Chapter A: Spin Degree of Freedom in a Ring Resonator}

In Chapter 3, we showed that a ring resonator can support two degenerate, the clockwise and the counterclockwise circulating, states. However, backscattering in the ring waveguides can lead to a coupling between the two modes. In this Chapter, we show that this coupling causes a splitting of the transmission spectrum of an ADF. We also discuss the effect of backscattering on the robustness of edge states.

We consider an add-drop filter and employ the single-mode approximation. But now, instead of a single mode, the ring has two degenerate modes, labelled by spin up and down. In the absence of any coupling between the modes, the time evolution for the ring energy amplitude $a_{\sigma}$ of a mode, for a plane wave excitation with frequency $\omega$, is given as (see Chapter 3)

$$
\frac{d a_{\sigma}}{d t}=-i\left(\omega-\omega_{0}\right) a_{\sigma}-\left(2 \kappa_{\mathrm{ex}}+\kappa_{\mathrm{in}}\right) a_{\sigma}-\sqrt{2 \kappa_{\mathrm{ex}}} \mathcal{E}_{\mathrm{I}}^{\sigma}
$$

Here, $\sigma= \pm$ represents the two spin states of the modes and $\mathcal{E}_{\mathrm{I}}^{\sigma}$ is the corresponding input field amplitude. For example, in Fig. A.1, input field at port 1 excites the counterclockwise circulating mode $(\sigma=+)$, while an excitation from port 2 results in a clockwise $(\sigma=-)$ circulating mode. For an ideal ring, the two modes coexist in the ring without any coupling. However, the dry etching step during the fabrication process (see Chapter 5) of an integrated ADF results in rough ring waveguide 
(a)

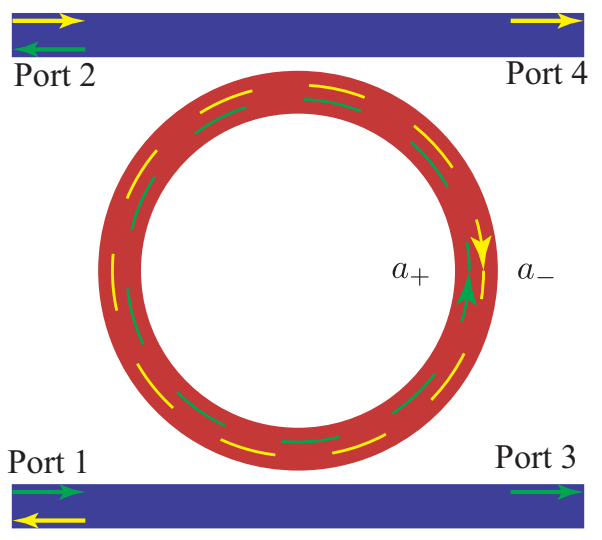

(b)

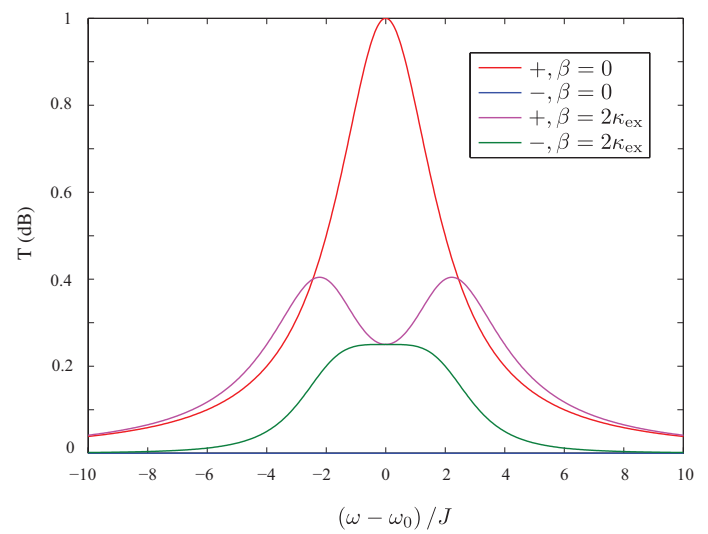

Figure A.1: (a) An ADF showing the two spin - the clockwise and counterclockwise rotating - modes. Input from port 1 excites the counterclockwise mode whereas input from port 2 excites the clockwise mode. (b) Transmission spectrum at the drop port (port 2 for CCW mode and port 1 for CW mode), with input only at port 1 , in the absence and presence of backscattering. In the absence of backscattering $(\beta=0)$, only the CCW mode exists in the ring. Presence of backscattering $\left(\beta=2 \kappa_{\text {ex }}\right)$ also excites the CW mode in the ring and hence the output at port 1 is nonzero. Also, the transmission spectrum splits.

surface, particularly the side walls of the waveguide. The surface roughness induces backscattering in the waveguide and hence, can lead to a coupling between the two modes. The steady-state solution for the two modes can then be written as

$$
\left(\begin{array}{cc}
i\left(\omega-\omega_{0}\right) & \beta \\
\beta & i\left(\omega-\omega_{0}\right)
\end{array}\right)\left(\begin{array}{c}
a_{+} \\
a_{-}
\end{array}\right)+\left(\begin{array}{cc}
2 \kappa_{\mathrm{ex}}+\kappa_{\mathrm{in}} & 0 \\
0 & 2 \kappa_{\mathrm{ex}}+\kappa_{\mathrm{in}}
\end{array}\right)\left(\begin{array}{c}
a_{+} \\
a_{-}
\end{array}\right)=-\sqrt{2 \kappa_{\mathrm{ex}}}\left(\begin{array}{c}
\mathcal{E}_{\mathrm{I}}^{+} \\
\mathcal{E}_{\mathrm{I}}^{-}
\end{array}\right)
$$

Here, the parameter $\beta$ is the coupling rate between the two modes. Fig. A.1(b) shows the transmission spectrum at the drop and through ports for this system with $\beta / \kappa_{\mathrm{ex}}=2$. As is evident from the spectrum, the coupling between the two spin states causes a splitting of the spectrum and the splitting, $\Delta \omega_{0}$, increases with $2 \beta$. In practice, the coupling rate $\beta$ is very small compared to $\kappa_{\text {ex }}$. The particularly high value of $\beta$ chosen here is just to exaggerate the splitting of the spectrum. 
(a)

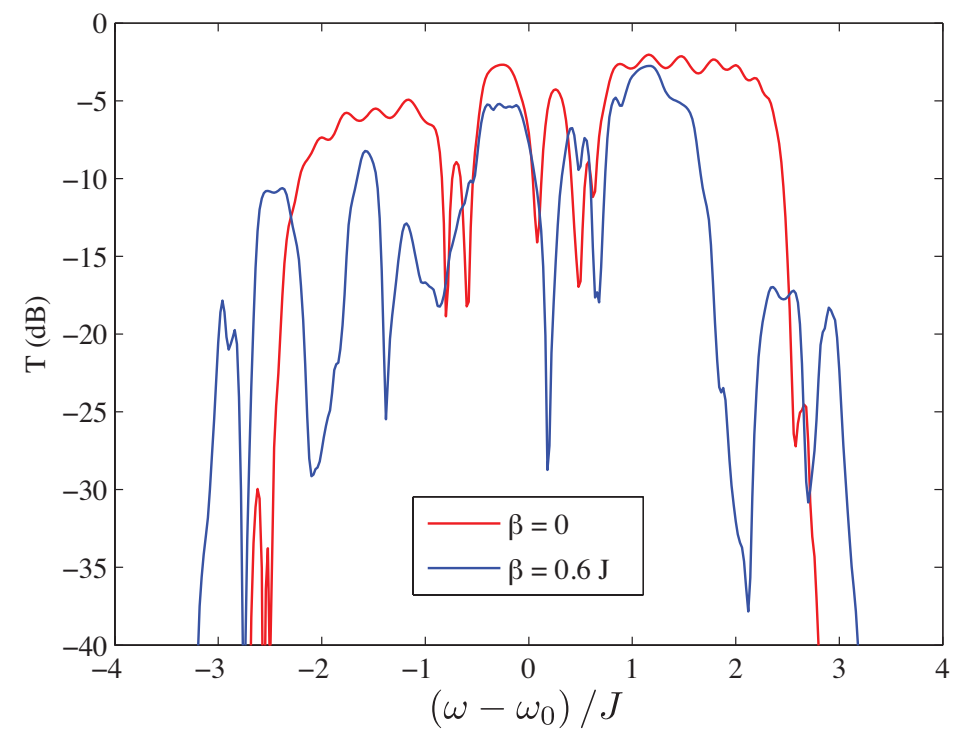

(b)

(c)

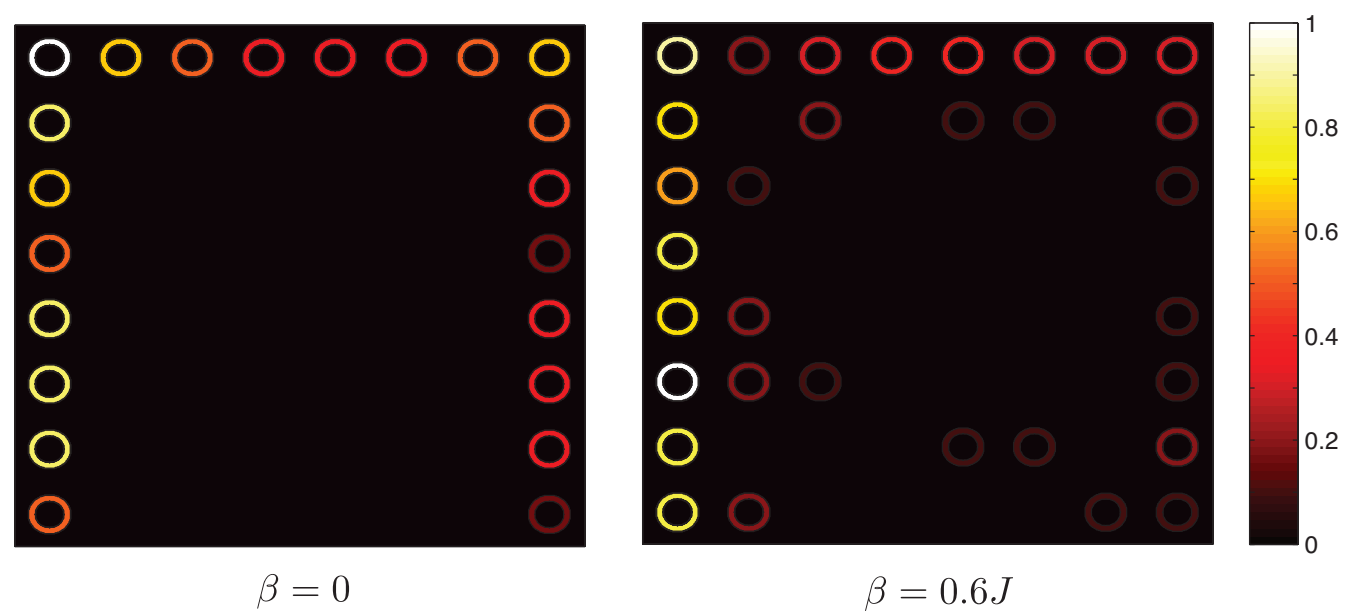

Figure A.2: (a) Transmission spectrum of a $8 \times 8$ lattice in the absence and presence of backscattering $(\beta=0.6 J)$. $(b, c)$ Ring intensity distributions at the long-edge for the two cases. Backscattering affects the robustness of edge states. 
We use a similar procedure to analyze the transmission of coupled ring resonators in the presence of backscattering. For a lattice with $N_{\mathrm{SR}}=N_{x} \times N_{y}$ rings, the Hilbert space is now of dimension $2 N_{\mathrm{SR}}$ and the corresponding Hamiltonian is a square matrix of dimension $2 N_{\mathrm{SR}}$. For example, for a $2 \times 2$ lattice, the ring energy amplitude vector is

$$
\boldsymbol{a}=\left[a_{+1}, a_{+2}, a_{+3}, a_{+4}, a_{-1}, a_{-2}, a_{-2}, a_{-4}\right]^{T},
$$

where we have labeled the rings following Fig. 4.10. The Hamiltonian for this system is then written as

$$
H_{0}=\left(\begin{array}{cccc|cccc}
\omega_{0} & -J & -J & 0 & -\beta & 0 & 0 & 0 \\
-J & \omega_{0} & 0 & -J & 0 & -\beta & 0 & 0 \\
-J & 0 & \omega_{0} & -J e^{i \alpha} & 0 & 0 & -\beta & 0 \\
0 & -J & -J e^{-i \alpha} & \omega_{0} & 0 & 0 & 0 & -\beta \\
\hline-\beta & 0 & 0 & 0 & \omega_{0} & -J & -J & 0 \\
0 & -\beta & 0 & 0 & -J & \omega_{0} & 0 & -J \\
0 & 0 & -\beta & 0 & -J & 0 & \omega_{0} & -J e^{-i \alpha} \\
0 & 0 & 0 & -\beta & 0 & -J & -J e^{i \alpha} & \omega_{0}
\end{array}\right) .
$$

In the absence of backscattering $(\beta=0)$, the system is thus equivalent to two independent spin systems. Moreover, the magnetic field for the two spin states is reversed indicated by the sign reversal in hopping phase along $x$-axis. The system therefore simulates a quantum spin-Hall effect. However, by appropriately choosing an input port, we can excite a particular spin state in the system.

The presence of backscattering $(\beta \neq 0)$ results in a mixing of the two spin states and affects the topological protection of the edge states. As an illustration, 
Fig. A.2 shows the transmission spectrum of an $8 \times 8$ lattice with $\beta=0,0.6 \mathrm{~J}$. The backscattering significantly distorts the spectrum, especially for the long edge. The ring intensity distribution for the two cases, at the long-edge, is also shown. In the presence of backscattering, the edge state is no longer confined to the edge of the lattice and instead, spreads to the bulk. The choice of $\beta=0.6 J$ was made to demonstrate the effect of backscattering. For our experimental system, we estimate $\beta / J$ to be 0.04 and hence, its effect is negligible. 


\section{Chapter B: Effect of Link Ring on Coupling Rate}

The implementation of a synthetic magnetic field for photons hinges on the specific placement of the link rings in our system. In Chapter 4, using a rigorous transfer matrix approach, we showed that when the link ring is anti-resonant with the site rings, i.e., when the length $L_{\mathrm{LR}}=L_{\mathrm{SR}}+\eta$, the link ring does not store any energy and hence, it acts only as a waveguide coupler, and not as a resonator. In this situation, the coupling rate between a link ring and its nearest neighbor is the same as that between the site ring and the link ring connecting the two site rings.

Here, we analyze a situation when the anti-resonant condition is not necessarily valid. We consider two site rings coupled by a link resonator (Fig. B.1). The site rings have a length $L_{\mathrm{SR}}$ and the link ring $L_{\mathrm{LR}}=L_{\mathrm{SR}}+\eta$. Furthermore, the link ring is vertically displaced by length $\xi$. We label the fields as shown in Fig. B.1.

(a)

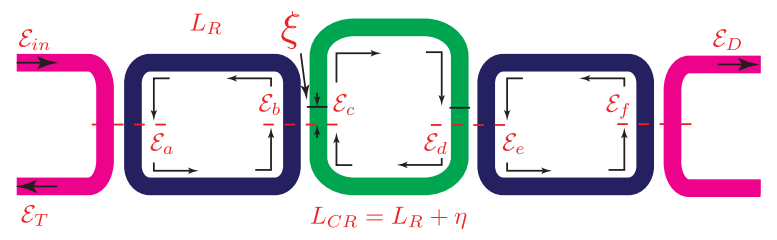

(b)

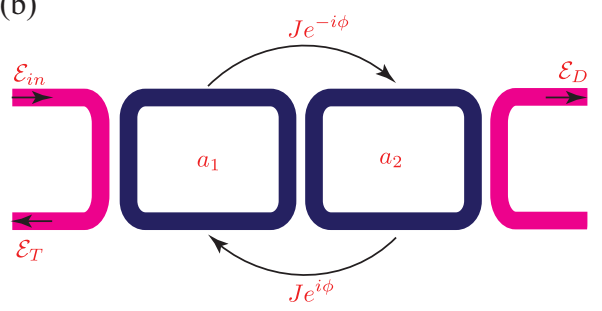

Figure B.1: (a) A system of two site rings, coupled by a link ring, and also coupled to input and output waveguides. The figure labels the fields for the transfer matrix analysis. (b) Description of the system in coupled mode theory. The effect of the link ring is contained in the coupling rate $J$ and hopping phase $\phi$. 
Using transfer matrix formalism (see Section 4.1), we can relate the different field amplitudes as

$$
\begin{aligned}
& \left(\begin{array}{c}
\mathcal{E}_{a} \\
\mathcal{E}_{b}
\end{array}\right)=\frac{1}{i \kappa_{\mathrm{I}, \mathrm{O}}}\left(\begin{array}{cc}
t_{\mathrm{I}, \mathrm{O}} & -1 \\
\frac{1}{\Phi_{\mathrm{R}}} & -\frac{t_{\mathrm{I}, \mathrm{O}}}{\Phi_{\mathrm{R}}}
\end{array}\right)\left(\begin{array}{c}
\mathcal{E}_{\mathrm{T}} \\
\mathcal{E}_{\mathrm{I}}
\end{array}\right), \\
& \left(\begin{array}{c}
\mathcal{E}_{c} \\
\mathcal{E}_{d}
\end{array}\right)=\frac{1}{i \kappa}\left(\begin{array}{cc}
t & -\Phi_{R} \\
\frac{1}{\Phi_{\mathrm{CRL}}} & -\frac{t \Phi_{\mathrm{R}}}{\Phi_{\mathrm{CRL}}}
\end{array}\right)\left(\begin{array}{c}
\mathcal{E}_{b} \\
\mathcal{E}_{a}
\end{array}\right) \text {, } \\
& \left(\begin{array}{c}
\mathcal{E}_{e} \\
\mathcal{E}_{f}
\end{array}\right)=\frac{1}{i \kappa}\left(\begin{array}{cc}
t & -\Phi_{\mathrm{CRU}} \\
\frac{1}{\Phi_{\mathrm{R}}} & -\frac{t \Phi_{\mathrm{CRU}}}{\Phi_{\mathrm{R}}}
\end{array}\right)\left(\begin{array}{c}
\mathcal{E}_{d} \\
\mathcal{E}_{c}
\end{array}\right) \\
& \left(\begin{array}{c}
\mathcal{E}_{\mathrm{D}} \\
0
\end{array}\right)=\frac{1}{i \kappa_{\mathrm{I}, \mathrm{O}}}\left(\begin{array}{cc}
t_{\mathrm{I}, \mathrm{O}} & -\Phi_{\mathrm{R}} \\
1 & -t_{\mathrm{I}, \mathrm{O}} \Phi_{\mathrm{R}}
\end{array}\right)\left(\begin{array}{c}
\mathcal{E}_{f} \\
\mathcal{E}_{e}
\end{array}\right)
\end{aligned}
$$

Here, $t_{\mathrm{I}, \mathrm{O}}$ and $k_{\mathrm{I}, \mathrm{O}}$ are the field transmission and cross coupling coefficients for coupling of link rings to the input and output ports, and $t, k$ are those for the coupling between the rings. $\Phi_{\mathrm{R}}=e^{-i \beta \frac{L_{\mathrm{SR}}}{2}} e^{-\alpha \frac{L_{\mathrm{SR}}}{2}}$ is the propagation term in the half length of the link rings. Similarly, $\Phi_{\mathrm{CRL}}=e^{-i \beta\left(\frac{L_{\mathrm{LR}}}{2}-2 \xi\right)} e^{-i \beta\left(\frac{L_{\mathrm{LR}}}{2}-2 \xi\right)}$ and $\Phi_{\mathrm{CRU}}=$ $e^{-i \beta\left(\frac{L_{\mathrm{LR}}}{2}+2 \xi\right)} e^{-i \beta\left(\frac{L_{\mathrm{LR}}}{2}+2 \xi\right)}$ are the propagation terms for the lower and upper arms of the link ring. These equations give the field at the drop port as $\mathcal{E}_{\mathrm{D}}=\frac{e^{-\frac{1}{2} \gamma\left(3 L_{\mathrm{SR}}+\eta-4 \xi\right)} \kappa^{2} \kappa_{\mathrm{I}, \mathrm{O}}^{2}}{1-e^{-\gamma\left(L_{\mathrm{SR}}+\eta\right)} t^{2}-e^{-\gamma\left(3 L_{\mathrm{SR}}+\eta\right)} t_{\mathrm{I}, \mathrm{O}}^{2}+2 e^{-\gamma\left(2 L_{\mathrm{SR}}+\eta\right)} t t_{\mathrm{I}, \mathrm{O}}-2 e^{-\gamma\left(L_{\mathrm{SR}}\right)} t t_{\mathrm{I}, \mathrm{O}}+e^{-\gamma\left(2 L_{\mathrm{SR}}\right)} t^{2} t_{\mathrm{I}, \mathrm{O}}^{2}}$,

where $\gamma=(\alpha+i \beta)$.

In the weak coupling limit, i.e., when $\kappa_{\mathrm{I}, \mathrm{O}}, \kappa \ll 1$ and $t_{\mathrm{I}, \mathrm{O}}, t \approx 1$, the expression 
for the drop field can be simplified to

$$
\mathcal{E}_{\mathrm{D}}=\frac{2 e^{i 2 \beta \xi} \kappa^{2} \kappa_{i o}^{2}}{2\left(2 \gamma L_{\mathrm{SR}}+\kappa_{i o}^{2}\right) \kappa^{2} \cos \left(\frac{\beta \eta}{2}\right)-i\left(\left(2 \gamma L_{\mathrm{SR}}+\kappa_{\mathrm{I}, \mathrm{O}}^{2}\right)^{2}+\kappa^{4}\right) \sin \left(\frac{\beta \eta}{2}\right)} .
$$

Here, we have assumed that the extra length $\eta$ of the link ring and its vertical shift $\xi$ are negligible compared to $L_{\mathrm{SR}}$, so that the loss incurred in these extra lengths is insignificant. In our experimental system, $\eta=320 \mathrm{~nm}, \xi=80 \mathrm{~nm}$ and $L_{\mathrm{SR}} \approx 70 \mu \mathrm{m}$, which justifies the above assumption.

Now, we analyze this system of two site rings coupled by a link ring using the coupled mode theory, where the coupling between the site rings can effectively be described by a coupling rate $J$ and a hopping phase $\pm \phi$ (Fig. B.1(b)). The effect of the link ring is captured in $J$ and $\phi$. The rate equations for the time evolution of the ring energy amplitudes, $a_{1}(t)$ and $a_{2}(t)$, are

$$
\begin{aligned}
\frac{d a_{1}}{d t} & =\left(-i \omega_{0}-\kappa_{\mathrm{ex}}-\kappa_{\mathrm{in}}\right) a_{1}-i J e^{-i \phi} a_{2}-\sqrt{2 \kappa_{\mathrm{ex}}} \mathcal{E}_{\mathrm{I}} \\
\frac{d a_{2}}{d t} & =\left(-i \omega_{0}-\kappa_{\mathrm{ex}}-\kappa_{\mathrm{in}}\right) a_{2}-i J e^{i \phi} a_{1}
\end{aligned}
$$

where $\kappa_{\mathrm{ex}}$ is the coupling of the site rings to the input and output waveguides and $\kappa_{\text {in }}$ is the resonator loss rate. A steady-state solution of the above equations for a plane wave excitation of frequency $\omega$ gives

$$
\begin{aligned}
& a_{1}=\frac{\left(i\left(\omega-\omega_{0}\right)-\kappa_{\mathrm{ex}}-\kappa_{\mathrm{in}}\right) \sqrt{2 \kappa_{\mathrm{ex}}} \mathcal{E}_{\mathrm{I}}}{\left(i\left(\omega-\omega_{0}\right)-\kappa_{\mathrm{ex}}-\kappa_{\mathrm{in}}\right)^{2}+J^{2}} \\
& a_{2}=\frac{i J e^{i \phi} a_{1}}{\left(i\left(\omega-\omega_{0}\right)-\kappa_{\mathrm{ex}}-\kappa_{\mathrm{in}}\right)} .
\end{aligned}
$$

Then, the field output at the drop port $\mathcal{E}_{D}^{\mathrm{CMT}}=\sqrt{2 \kappa_{\mathrm{ex}}} a_{2}$ is

$$
\mathcal{E}_{\mathrm{D}}^{\mathrm{CMT}}=\frac{i 2 e^{i \phi} J \kappa_{\mathrm{ex}} \mathcal{E}_{\mathrm{I}}}{\left(i\left(\omega-\omega_{0}\right)-\kappa_{\mathrm{ex}}-\kappa_{\mathrm{in}}\right)^{2}+J^{2}} .
$$


To compare this expression for the drop field to that derived using the transfer matrix approach, we use the relations derived in Chapter 3,

$$
\begin{aligned}
\beta & =\frac{\left(\omega_{0}-\omega\right)}{v_{\mathrm{g}}} \\
\kappa_{\mathrm{in}} & =\alpha v_{\mathrm{g}} \\
\kappa_{\mathrm{ex}} & =\frac{\kappa_{\mathrm{I}, \mathrm{O}}^{2}}{2} \frac{v_{\mathrm{g}}}{L_{\mathrm{SR}}} \\
J & =\frac{\kappa^{2}}{2} \frac{v_{\mathrm{g}}}{L_{\mathrm{SR}}} .
\end{aligned}
$$

The drop field in the transfer matrix formulation is then

$$
\mathcal{E}_{\mathrm{D}}=\frac{2 e^{i \phi} J \kappa_{\mathrm{ex}}}{-2 J\left(i\left(\omega-\omega_{0}\right)-\kappa_{\mathrm{ex}}-\kappa_{\mathrm{in}}\right) \cos \left(\frac{\beta \eta}{2}\right)-i\left(J^{2}+\left(i\left(\omega-\omega_{0}\right)-\kappa_{\mathrm{ex}}-\kappa_{\mathrm{in}}\right)^{2}\right) \sin \left(\frac{\beta \eta}{2}\right)} \mathcal{E}_{I},
$$

where $\phi=2 \beta \xi$. We see that for $\beta \eta=(\pi, 3 \pi, 5 \pi, \ldots)$, i.e., when the link ring is anti-resonant to the site rings, the two expressions are identical. Because the link ring is anti-resonant, it does not store any energy and simply acts as a waveguide, without affecting the coupling between the site rings.

When $\beta \eta$ is not an odd-integer multiple of $\pi$, the two expressions for the drop field, derived using the coupled mode theory and the transfer matrix method, are identical if we use an effective coupling rate $J^{\text {eff }}=J / \sin \left(\frac{\beta \eta}{2}\right)$ and shift the resonance frequency of the site rings as $\omega_{0}^{\mathrm{eff}}=\omega_{0}+J \cot \left(\frac{\beta \eta}{2}\right)$. 


\section{Bibliography}

[1] von Klitzing, K., Dorda, G. \& Pepper, M. New method for high-accuracy determination of the fine-structure constant based on quantized Hall resistance. Phys. Rev. Lett. 45, 494-497 (1980).

[2] Tsui, D. \& Gossard, A. Resistance standard using quantization of the Hall resistance of GaAs-AlxGa1xAs heterostructures. Appl. Phys. Lett. 38, 550552 (1981).

[3] Tsui, D., Stormer, H. \& Gossard, A. Two-dimensional magnetotransport in the extreme quantum limit. Phys. Rev. Lett. 48, 1559-1562 (1982).

[4] Paalanen, M., Tsui, D. \& Gossard, A. Quantized Hall effect at low temperatures. Phys. Rev. B 25, 5566-5569 (1982).

[5] Wen, X.-G. Quantum Field Theory of Many-body Systems (Oxford University Press, USA, 2007).

[6] Lagendijk, A., van Tiggelen, B. \& Wiersma, D. S. Fifty years of Anderson localization. Phys. Today 62, 24 (2009).

[7] Halperin, B. I. Quantized Hall Conductance, Current Carrying Edge States and Extended States in 2D Disordered Potential. Phys. Rev. B 25, 2185-2190 (1982).

[8] Hatsugai, Y. Chern number and edge states in the integer quantum Hall effect. Phys. Rev. Lett. 71, 3697-3700 (1993).

[9] Haldane, F. \& Raghu, S. Possible realization of directional optical waveguides in photonic crystals with broken time-reversal symmetry. Phys. Rev. Lett. 100, 013904 (2008).

[10] Raghu, S. \& Haldane, F. Analogs of quantum-Hall-effect edge states in photonic crystals. Phys. Rev. A 78, 033834 (2008). 
[11] Wang, Z., Chong, Y., Joannopoulos, J. \& Soljačić, M. Reflection-free one-way edge modes in a gyromagnetic photonic crystal. Phys. Rev. Lett. 100, 013905 (2008).

[12] Wang, Z., Chong, Y., Joannopoulos, J. \& Soljačić, M. Observation of unidirectional backscattering-immune topological electromagnetic states. Nature 461, 772-775 (2009).

[13] Liu, K., Shen, L. \& He, S. One-way edge mode in a gyromagnetic photonic crystal slab. Opt. Lett. 37, 4110 (2012).

[14] Khanikaev, A. B. et al. Photonic topological insulators. Nat. Mater. 12, 233-9 (2013).

[15] Hafezi, M., Demler, E., Lukin, M. \& Taylor, J. Robust optical delay lines with topological protection. Nat. Phys. 7, 907-912 (2011).

[16] Hatsugai, Y. Topological aspects of the quantum Hall effect. J. Phys. Condens. Matter 9, 2507-2549 (1997).

[17] Rechtsman, M. \& Zeuner, J. Strain-induced pseudomagnetic field and Landau levels in photonic structures. Nat. Photonics 7, 153-158 (2012).

[18] Plotnik, Y. et al. Observation of unconventional edge states in 'photonic graphene'. Nat. Mater. 13, 57-62 (2014).

[19] Rechtsman, M. C. et al. Photonic Floquet topological insulators. Nature (2013).

[20] Fang, K., Yu, Z. \& Fan, S. Photonic Aharonov-Bohm effect based on dynamic modulation. Phys. Rev. Lett. 108, 153901 (2012).

[21] Lira, H., Yu, Z., Fan, S. \& Lipson, M. Electrically driven nonreciprocity induced by interband photonic pransition on a silicon chip. Phys. Rev. Lett. 109, 033901 (2012).

[22] Fang, K., Yu, Z. \& Fan, S. Experimental demonstration of a photonic Aharonov-Bohm effect at radio frequencies. Phys. Rev. B 87, 060301 (2013).

[23] Wiersma, D. S., Bartolini, P., Lagendijk, A. \& Righini, R. Localization of light in a disordered medium. Nature 390, 671-673 (1997).

[24] Genack, A., Sebbah, P., Stoytchev, M. \& van Tiggelen, B. Statistics of wave dynamics in random media. Phys. Rev. Lett. 82, 715-718 (1999).

[25] Chabanov, A. \& Genack, A. Photon localization in resonant media. Phys. Rev. Lett. 87, 153901 (2001).

[26] Segev, M., Silberberg, Y. \& Christodoulides, D. N. Anderson localization of light. Nat. Photonics 7, 197-204 (2013). 
[27] Thouless, D. \& Kohmoto, M. Quantized Hall conductance in a twodimensional periodic potential. Phys. Rev. Lett. 49, 405-408 (1982).

[28] Kohmoto, M. Topological invariant and the quantization of the Hall conductance. Ann. Phys. (N. Y). 160, 343-354 (1985).

[29] Hafezi, M. Synthetic gauge field with photons . Int. J. Mod. Phys. B 28, 7-9 (2014).

[30] Ando, T., Matsumoto, Y. \& Uemura, Y. Theory of Hall effect in a twodimensional electron system. J. Phys. Soc. Japan 39, 279-288 (1975).

[31] Laughlin, R. Quantized Hall conductivity in two dimensions. Phys. Rev. B 23, 5632-5633 (1981).

[32] Niu, Q., Thouless, D. \& Wu, Y. Quantized Hall conductance as a topological invariant. Phys. Rev. B 31, 3372-3377 (1985).

[33] Aoki, H. Effect of Localization on the Hall Conducitcity in the TwoDimensional System in Strong Magnetic Fields. Solid 38, 1079-1082 (1981).

[34] Thouless, D. Localisation and the two-dimensional Hall effect. J. Phys. C Solid State Phys. 14, 3475-3480 (1981).

[35] Avishai, Y., Hatsugai, Y. \& Kohmoto, M. Persistent currents and edge states in a magnetic field. Phys. Rev. B 47, 9501-9512 (1993).

[36] Willett, R., Eisenstein, J. \& Störmer, H. Observation of an even-denominator quantum number in the fractional quantum Hall effect. Phys. Rev. Lett. 59, 1776-1779 (1987).

[37] Chakraborty, T. \& Pietiläinen, P. The Quantum Hall Effects (Springer, 1995), 2 edn.

[38] Yoshioka, D. The Quantum Hall Effect (Springer, 2002).

[39] Hofstadter, D. Energy levels and wave functions of Bloch electrons in rational and irrational magnetic fields. Phys. Rev. B 14, 2239-2249 (1976).

[40] Haldane, F. Model for a quantum Hall effect without Landau levels: Condensed-matter realization of the"parity anomaly". Phys. Rev. Lett. 61, 2015-2018 (1988).

[41] Yariv, A. Universal relations for coupling between microring resonators and waveguides. Electron. Lett. 36, 321-322 (2000).

[42] Mookherjea, S. Spectral characteristics of coupled resonators. J. Opt. Soc. Am. B 23, 1137 (2006). 
[43] Little, B., Chu, S., Haus, H., Foresi, J. \& Laine, J.-P. Microring resonator channel dropping filters. J. Light. Technol. 15, 998-1005 (1997).

[44] Gardiner, C. W. \& Collett, M. J. Input and output in damped quantum systems: Quantum stochastic differential equations and the master equation. Phys. Rev. A 31, 3761-3774 (1985).

[45] Chremmos, I. \& Uzunoglu, N. Modes of the infinite square lattice of coupled microring resonators. JOSA A 25, 3043-3050 (2008).

[46] Sinova, J. et al. Universal Intrinsic Spin Hall Effect. Phys. Rev. Lett. 92, 126603 (2004).

[47] König, M. et al. The Quantum Spin Hall Effect: Theory and Experiment. J. Phys. Soc. Japan 77, 031007 (2008).

[48] Xia, F., Sekaric, L. \& Vlasov, Y. Ultracompact optical buffers on a silicon chip. Nat. Photonics 1, 65-71 (2007).

[49] Melloni, A. et al. Tunable delay lines in silicon photonics: coupled resonators and photonic crystals, a comparison. IEEE Photonics J. 2 (2010).

[50] Liu, H.-C. \& Yariv, A. "Ideal" optical delay lines based on tailored-coupling and reflecting, coupled-resonator optical waveguides. Opt. Lett. 37, 1964-6 (2012).

[51] Yariv, A., Xu, Y., Lee, R. K. \& Scherer, A. Coupled-resonator optical waveguide: a proposal and analysis. Opt. Lett. 24, 711-3 (1999).

[52] Poon, J. et al. Matrix analysis of microring coupled-resonator optical waveguides. Opt. Express 12, 90-103 (2004).

[53] Mookherjea, S. Dispersion characteristics of coupled-resonator optical waveguides. Opt. Lett. 30, 2406-2408 (2005).

[54] Notomi, M., Kuramochi, E. \& Tanabe, T. Large-scale arrays of ultrahigh-Q coupled nanocavities. Nat. Photonics 2, 741-747 (2008).

[55] Cooper, M. L. et al. Statistics of light transport in 235-ring silicon coupledresonator optical waveguides. Opt. Express 18, 26505-16 (2010).

[56] Ferrari, C., Morichetti, F. \& Melloni, A. Disorder in coupled-resonator optical waveguides. J. Opt. Soc. Am. B 26, 858 (2009).

[57] Mookherjea, S. \& Schneider, M. a. Avoiding bandwidth collapse in long chains of coupled optical microresonators. Opt. Lett. 36, 4557-9 (2011).

[58] Mookherjea, S. \& Oh, A. Effect of disorder on slow light velocity in optical slow-wave structures. Opt. Lett. 32, 289-291 (2007). 
[59] Topolancik, J., Ilic, B. \& Vollmer, F. Experimental observation of strong photon localization in disordered photonic crystal waveguides. Phys. Rev. Lett. 99, 253901 (2007).

[60] Lahini, Y. et al. Anderson localization and nonlinearity in one-dimensional disordered photonic lattices. Phys. Rev. Lett. 100, 013906 (2008).

[61] Tsay, A. \& Van, V. Analytic theory of strongly-coupled microring resonators. Quantum Electron. IEEE J. 47, 997-1005 (2011).

[62] Absil, P. P. et al. Wavelength conversion in GaAs micro-ring resonators. Opt. Lett. 25, 554-6 (2000).

[63] Almeida, V., Barrios, C., Panepucci, R. \& Lipson, M. All-optical control of light on a silicon chip. Nature 431, 1081-1084 (2004).

[64] Ferrera, M. et al. On-chip CMOS-compatible all-optical integrator. Nat. Commun. 1, 29 (2010).

[65] Wang, T.-J., Chu, C.-H. \& Lin, C.-Y. Electro-optically tunable microring resonators on lithium niobate. Opt. Lett. 32, 2777-9 (2007).

[66] Barwicz, T. et al. Microring-resonator-based add-drop filters in SiN: fabrication and analysis. Opt. Express 12, 1437-42 (2004).

[67] Vlasov, Y. \& McNab, S. Losses in single-mode silicon-on-insulator strip waveguides and bends. Opt. Express 12, 1622-31 (2004).

[68] Xu, Q., Fattal, D. \& Beausoleil, R. G. Silicon microring resonators with 1.5-micron radius. Opt. Express 16, 4309-15 (2008).

[69] Dumon, P. \& Bogaerts, W. Low-loss SOI photonic wires and ring resonators fabricated with deep UV lithography. IEEE Photonics Technol. Lett. 16, 1328-1330 (2004).

[70] Barwicz, T. \& Popović, M. Fabrication of add-drop filters based on frequencymatched microring resonators. J. Light. Technol. 24, 2207-2218 (2006).

[71] Bojko, R. J. et al. Electron beam lithography writing strategies for low loss, high confinement silicon optical waveguides. J. Vac. Sci. Technol. B Microelectron. Nanom. Struct. 29, 06F309 (2011).

[72] Bogdanov, A. L., Lapointe, J. \& Schmid, J. H. Electron-beam lithography for photonic waveguide fabrication: measurement of the effect of field stitching errors on optical performance and evaluation of a new compensation method. J. Vac. Sci. Technol. B Microelectron. Nanom. Struct. 30, 031606 (2012).

[73] Lim, M., Murphy, T., Ferrera, J., Damask, J. N. \& Smith, H. I. Fabrication techniques for grating-based optical devices. J. Vac. Sci. Technol. B Microelectron. Nanom. Struct. 17, 3208-3211 (1999). 
[74] Lipson, M. Guiding, modulating, and emitting light on silicon-challenges and opportunities. J. Light. Technol. 23, 4222-4238 (2005).

[75] Almeida, V. R., Panepucci, R. R. \& Lipson, M. Nanotaper for compact mode conversion. Opt. Lett. 28, 1302-4 (2003).

[76] Tokushima, M., Kamei, A. \& Horikawa, T. Dual-tapered 10- $\mu$-spot-size converter with double core for coupling polarization-independent silicon rib waveguides to single-mode optical fibers. Appl. Phys. Express 5, 022202 (2012).

[77] Laere, F. V. \& Claes, T. Compact focusing grating couplers for silicon-oninsulator integrated circuits. IEEE Photonics Technol. Lett. 19, 1919-1921 (2007).

[78] Taillaert, D. et al. Grating couplers for coupling between optical fibers and nanophotonic waveguides. Jpn. J. Appl. Phys. 45, 6071-6077 (2006).

[79] Vermeulen, D. et al. High-efficiency fiber-to-chip grating couplers realized using an advanced CMOS-compatible silicon-on-insulator platform. Opt. Express 18, 18278-18283 (2010).

[80] Gifford, D., Soller, B., Wolfe, M. \& Froggatt, M. Optical vector network analyzer for single-scan measurements of loss, group delay, and polarization mode dispersion. Appl. Opt. 44, 7282-7286 (2005).

[81] Hafezi, M., Mittal, S., Fan, J., Migdall, A. \& Taylor, J. M. Imaging topological edge states in silicon photonics. Nat. Photonics 7, 1001-1005 (2013).

[82] Mittal, S. et al. Topologically robust transport of photons in a synthetic gauge field. Phys. Rev. Lett. 113, 087403 (2014).

[83] Texier, C. \& Comtet, A. Universality of the Wigner time delay distribution for one-dimensional random potentials. Phys. Rev. Lett. 82, 4220-4223 (1999).

[84] Chabanov, A. \& Genack, A. Statistics of dynamics of localized waves. Phys. Rev. Lett. 87, 233903 (2001).

[85] van Tiggelen, B. a., Sebbah, P., Stoytchev, M. \& Genack, a. Z. Delay-time statistics for diffuse waves. Phys. Rev. E. Stat. Phys. Plasmas. Fluids. Relat. Interdiscip. Topics 59, 7166-72 (1999).

[86] Pendry, J. Quasi-extended electron states in strongly disordered systems. J. Phys. C Solid State Phys. 20, 733-742 (1987).

[87] Schwartz, T., Bartal, G., Fishman, S. \& Segev, M. Transport and Anderson localization in disordered two-dimensional photonic lattices. Nature 446, 52-5 (2007). 
[88] Mookherjea, S., Park, J. S., Yang, S.-H. \& Bandaru, P. R. Localization in silicon nanophotonic slow-light waveguides. Nat. Photonics 2, 90-93 (2008).

[89] Atlasov, K. A. et al. 1D photonic band formation and photon localization in finite-size photonic-crystal waveguides. Opt. Express 18, 117-22 (2010).

[90] Hu, W., Pillay, J., Wu, K., Pasek, M. \& Chong, Y. Measurement of a topological edge invariant in a microwave network. arXiv 1408.1808 (2014). 1408.1808 .

[91] Kraus, Y., Lahini, Y., Ringel, Z., Verbin, M. \& Zilberberg, O. Topological states and adiabatic pumping in quasicrystals. Phys. Rev. Lett. 109, 106402 (2012).

[92] Priem, G. et al. Optical bistability and pulsating behaviour in Silicon-OnInsulator ring resonator structures. Opt. Express 13, 9623-8 (2005).

[93] Lin, Q., Painter, O. J. \& Agrawal, G. P. Nonlinear optical phenomena in silicon waveguides: modeling and applications. Opt. Express 15, 16604-44 (2007).

[94] Foster, M., Turner, A., Lipson, M. \& Gaeta, A. Nonlinear optics in photonic nanowires. Opt. Express 16, 1070-1078 (2008).

[95] Leuthold, J., Koos, C. \& Freude, W. Nonlinear silicon photonics. Nat. Photonics 4, 535-544 (2010).

[96] Barclay, P., Srinivasan, K. \& Painter, O. Nonlinear response of silicon photonic crystal microresonators excited via an integrated waveguide and fiber taper. Opt. Express 13, 801-20 (2005).

[97] Martinez, L. \& Lipson, M. High confinement suspended micro-ring resonators in silicon-on-insulator. Opt. Express 14, 6259-63 (2006).

[98] Chen, Y. \& Blair, S. Nonlinearity enhancement in finite coupled-resonator slow-light waveguides. Opt. Express 12, 3353-66 (2004).

[99] Matsuda, N. et al. A monolithically integrated polarization entangled photon pair source on a silicon chip. Sci. Rep. 2, 817 (2012).

[100] Takesue, H. Entangled photon pair generation using silicon wire waveguides. IEEE J. Quantum Electron. 18, 1722-1732 (2012).

[101] Davanco, M. et al. Telecommunications-band heralded single photons from a silicon nanophotonic chip. Appl. Phys. Lett. 100, 261104 (2012).

[102] Fukuda, H. et al. Four-wave mixing in silicon wire waveguides. Opt. Express 13, 4629-37 (2005). 
[103] Melloni, A., Morichetti, F. \& Martinelli, M. Four-wave mixing and wavelength conversion in coupled-resonator optical waveguides. J. Opt. Soc. Am. B 25, C87 (2008).

[104] Ong, J. et al. Low-power continuous-wave four-wave mixing in silicon coupledresonator optical waveguides. Opt. Lett. 36, 2964-2966 (2011).

[105] Morichetti, F. et al. Travelling-wave resonant four-wave mixing breaks the limits of cavity-enhanced all-optical wavelength conversion. Nat. Commun. 2, 296 (2011).

[106] Manolatou, C. \& Lipson, M. All-optical silicon modulators based on carrier injection by two-photon absorption. J. Light. Technol. 24, 1433-1439 (2006).

[107] Fan, L. et al. An all-silicon passive optical diode. Science 335, 447-50 (2012).

[108] Aidelsburger, M. et al. Realization of the hofstadter hamiltonian with ultracold atoms in optical lattices. Phys. Rev. Lett. 111, 185301 (2013).

[109] Hunt, B. et al. Massive Dirac fermions and Hofstadter butterfly in a van der Waals heterostructure. Science 340, 1427-30 (2013).

[110] Dean, C. R. et al. Hofstadter's butterfly and the fractal quantum Hall effect in moiré superlattices. Nature 497, 598-602 (2013).

[111] Lahini, Y., Bromberg, Y., Christodoulides, D. N. \& Silberberg, Y. Quantum correlations in two-particle anderson localization. Phys. Rev. Lett. 105, 163905 (2010).

[112] Peruzzo, A. et al. Quantum walks of correlated photons. Science 329, 15001503 (2010).

[113] Poulios, K. et al. Quantum walks of correlated photon pairs in two-dimensional waveguide arrays. Phys. Rev. Lett. 112, 143604 (2014). 